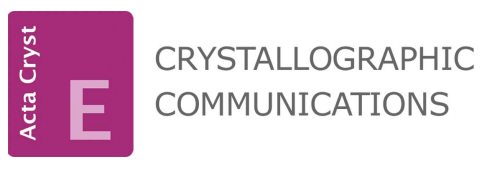

ISSN 2056-9890

Received 4 February 2016

Accepted 9 March 2016

Edited by S. Parkin, University of Kentucky, USA

Keywords: crystal structure; nickel; $\mathrm{H}_{2}$ pqd; $\alpha-\beta$ dioxime; trinuclear; hydrogen bonding.

CCDC references: $1462814 ; 1462813$

Supporting information: this article has supporting information at journals.iucr.org/e

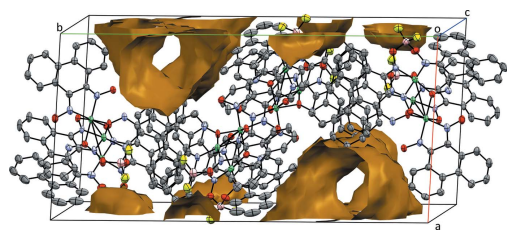

OPEN $\odot$ ACCESS

\section{Trinuclear nickel coordination complexes of phenanthrene-9,10-dione dioxime}

\author{
Owen M. Williams and Alan H. Cowley*
}

105 E. 24th St., Austin, TX 78704, USA. *Correspondence e-mail: acowley@cm.utexas.edu

A trinuclear nickel complex of phenanthrene-9,10-dione dioxime $\left(\mathrm{H}_{2} \mathrm{pqd}\right)$, namely bis $\left[\mu_{2}-9,10\right.$-bis(oxidoimino)phenanthrene $]$ bis $\left[\mu_{2}-10\right.$-(oxidoimino)phenanthrene-9-one oxime](phenanthrene-9,10-dione dioxime)trinickel(II) toluene disolvate, $\left[\mathrm{Ni}_{3}\left(\mathrm{C}_{14} \mathrm{H}_{8} \mathrm{~N}_{2} \mathrm{O}_{2}\right)_{2}\left(\mathrm{C}_{14} \mathrm{H}_{9} \mathrm{~N}_{2} \mathrm{O}_{2}\right)_{2}\left(\mathrm{C}_{14} \mathrm{H}_{10} \mathrm{~N}_{2} \mathrm{O}_{2}\right)\right] \cdot 2 \mathrm{C}_{7} \mathrm{H}_{8}$, has been isolated and its crystal structure determined. This complex features three independent $\mathrm{Ni}^{\mathrm{II}}$ atoms that are arranged in a triangular fashion along with five supporting ligands. There are two square-planar $\mathrm{Ni}^{\mathrm{II}}$ atoms and a third pseudooctahedral $\mathrm{Ni}^{\mathrm{II}}$ atom. While the square-planar $\mathrm{Ni}^{\mathrm{II}}$ atoms are stacked, there are no ligand bridges between them. Each square-planar $\mathrm{Ni}^{\mathrm{II}}$ atom, however, bridges with the pseudo-octahedral $\mathrm{Ni}^{\mathrm{II}}$ atom through $\mathrm{Ni}-\mathrm{N}-\mathrm{O}-\mathrm{Ni}$ and $\mathrm{Ni}-$ $\mathrm{O}-\mathrm{Ni}$ bonds. A fluoridoboration reaction of the proton-bridged species gave the analogous complex bis( $\mu_{2}$-bis $\{[10$-(oxidoimino)-9,10-dihydrophenanthren9-ylidene]amino\}difluoridoborato)(phenanthrene-9,10-dione dioxime)trinickel(II) dichloromethane trisolvate, $\left[\mathrm{Ni}_{3}\left(\mathrm{C}_{28} \mathrm{H}_{16} \mathrm{BF}_{2} \mathrm{~N}_{4} \mathrm{O}_{4}\right)_{2}\left(\mathrm{C}_{14} \mathrm{H}_{10} \mathrm{~N}_{2} \mathrm{O}_{2}\right)\right]$ $3 \mathrm{CH}_{2} \mathrm{Cl}_{2}$, which shows the same binding structure, but features a widened $\mathrm{Ni}-$ $\mathrm{Ni}$ interaction between the square-planar $\mathrm{Ni}^{\mathrm{II}}$ atoms. The proton-bridged complex completes the macrocyclic coordination around the square-planar $\mathrm{Ni}^{\mathrm{II}}$ atoms by means of an $\mathrm{O}-\mathrm{H}$. . O hydrogen bond. Both compounds feature $\mathrm{O}-$ $\mathrm{H} \cdot \mathrm{N}$ hydrogen bonds between the oxime and the $\mathrm{N}$ atoms attached to squareplanar nickel atom. The nickel units show no direct interaction with their nearest neighbors in the extended lattice. Two $\pi$-stacking interactions between adjacent molecules are found: one with a centroid-centroid distance of 3.886 (2) $\AA$ and the other with a centroid-centroid distance of 4.256 (3) $\AA$. In the latter case, although not aromatic, the distance to the centroid of the central phenanthrene ring is shorter, with a distance of 3.528 (3) $\AA$. Toluene molecules occupy the solvent channels that are oriented along the $c$ axis. In the fluoridoboronate structure, the solvent (DCM) was too badly disordered to be modelled, so its contribution was removed using SQUEEZE [Spek (2015). Acta Cryst. C71, 9-18].

\section{Chemical context}

Oxime functional groups can coordinate to transition metal ions in a variety of ways, due to the presence of both nitrogen and oxygen donors. On account of the multitude of possible coordinations, these ligands, and particularly $\alpha-\beta$ dioximes, have the capability of forming bridging multinuclear complexes with many transition metals, including nickel (Chaudhuri, 2003). From the standpoint of single-molecule magnets, these multi-nuclear complexes play an important role due to their ability to facilitate spin-frustration in magnetic transition-metal clusters (Aromí \& Brechin, 2006). Other nickel polynuclear compounds supported by oxime ligands have been reported (Jiang et al., 2005; Biswas et al., 2009). 
Phenanthrenequinone dioxime ( $\mathrm{pqdH}_{2}$ ) is an $\alpha-\beta$ dioxime ligand that incorporates a constrained ring system. Similar to other dioximes, however, it exists as three separate stereoisomers $(E-E, E-Z$, and $Z-Z)$, as confirmed by liquid chromatography - mass spectrometry. Interestingly, although this compound was synthesized over 100 years ago (Schmidt \& Söll, 1907), no coordination complexes of this ligand have been structurally characterized to date.

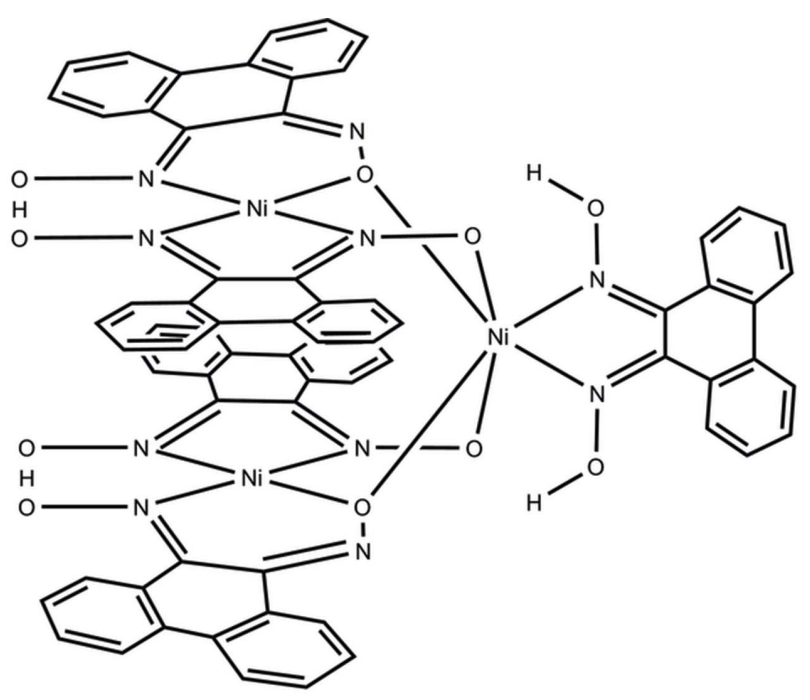

(1)

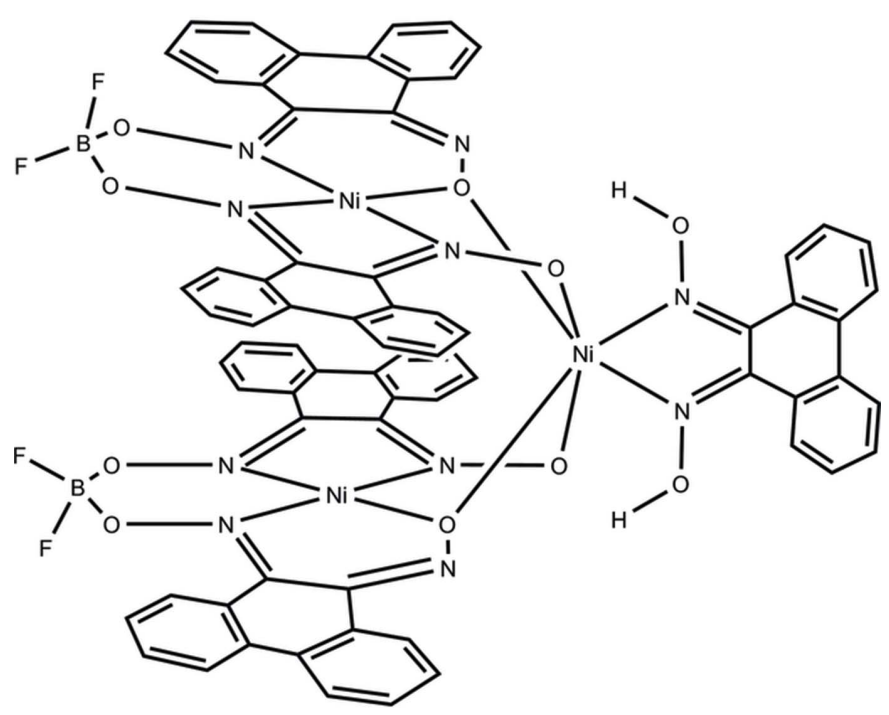

(2)

\section{Structural commentary}

Fig. 1 shows the structure of $\left[\mathrm{Ni}_{3}\left(\mathrm{H}_{2}\right.\right.$ pqd $\left.)(\mathrm{Hpqd})_{2}(\mathrm{pqd})_{2}\right],(\mathbf{1})$. This complex consists of three $\mathrm{Ni}^{\mathrm{II}}$ atoms in a triangular arrangement, two of which are in a square-planar coordination environment, while the third is in a pseudo-octahedral coordination environment. The square-planar $\mathrm{Ni}^{\mathrm{iI}}$ atoms (Ni1 and $\mathrm{Ni2}$ ) consist of one $\mathrm{N}, \mathrm{N}$-coordinating and one $\mathrm{N}, \mathrm{O}$-coordinating ligand. These ligands form bridges with the pseudooctahedral $\mathrm{Ni}^{\mathrm{II}}$ atom (Ni3) by means of their oxime $\mathrm{O}$ atoms.

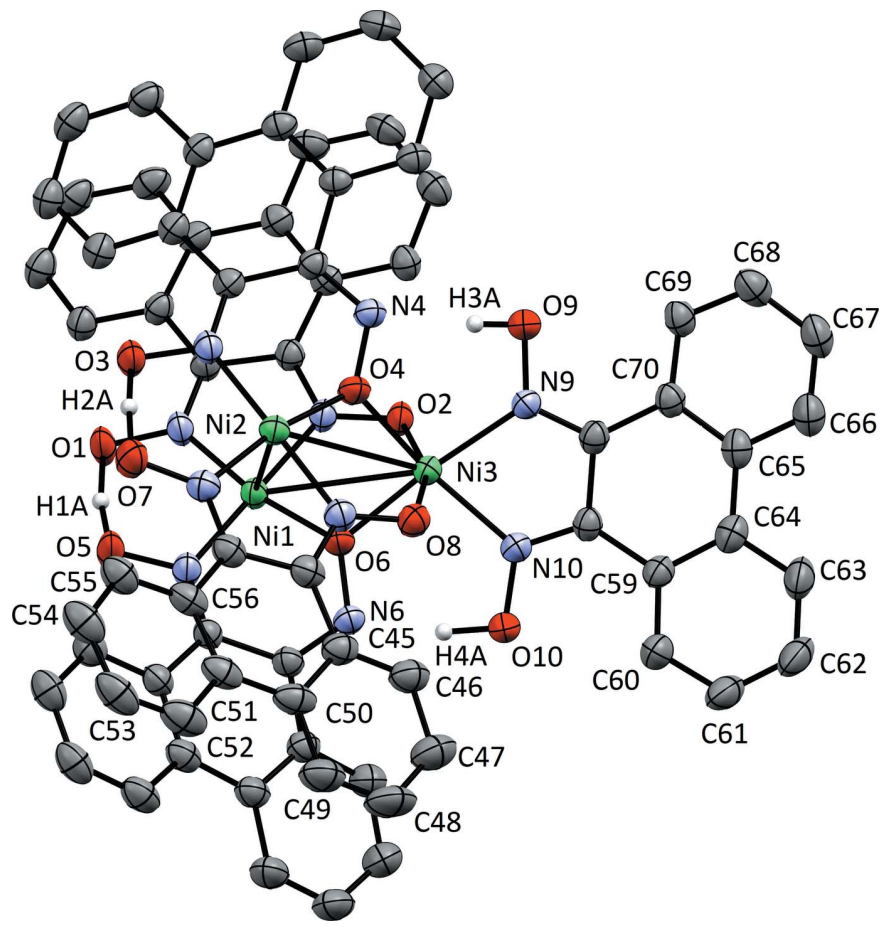

Figure 1

Displacement ellipsoid plot at the $50 \%$ probability level for $\left[\mathrm{Ni}_{3}\left(\mathrm{H}_{2}\right.\right.$ pqd $\left.)(\mathrm{Hpqd})_{2}(\mathrm{pqd})_{2}\right] . \mathrm{H}$ atoms (with the exception of hydrogenbonded atoms) and solvent molecules have been omitted for clarity.

This arrangement permits the formation of $\mathrm{Ni}-\mathrm{N}-\mathrm{O}-\mathrm{Ni}$ and $\mathrm{Ni}-\mathrm{O}-\mathrm{Ni}$ bridges between each square-planar $\mathrm{Ni}^{\mathrm{II}}$ atom and the pseudo-octahedral $\mathrm{Ni}^{\mathrm{II}}$ atom.

The structural features of the core ligation sphere warrant special attention. The $\mathrm{Ni}_{\mathrm{sp}}-\mathrm{Ni}_{\mathrm{sp}}$ distance is 3.3657 (9) $\AA$, a distance that precludes the presence of any metal-metal bonding. However, the distances between each of these nickel moieties and the pseudo-octahedral $\mathrm{Ni}^{\mathrm{II}}$ atom are nearly identical $\left[\mathrm{Ni}_{\mathrm{sp}}-\mathrm{Ni}_{\text {oct }}=3.2697\right.$ (7), 3.2674 (7) $\AA$ ] . The pseudooctahedral nickel geometry deviates significantly from a perfect octahedral symmetry $[\mathrm{O} 2-\mathrm{Ni3}-\mathrm{O} 8=160.00(9)$, $\left.\mathrm{O} 4-\mathrm{Ni3}-\mathrm{N} 10=164.8(1), \mathrm{N} 9-\mathrm{Ni3}-\mathrm{O} 6=165.5(1)^{\circ}\right]$.

Fig. 2 shows the complete coordination geometry of the compound $\left[\mathrm{Ni}_{3}\left(\mathrm{pqdH}_{2}\right)\left(\mathrm{pqdBF}_{2}\right)_{2}(\mathrm{pqd})_{2}\right]$, (2). The physical arrangement of the ligation sphere directly mimics that of (1). In this case, however, the steric bulk of the $\mathrm{BF}_{2}$ groups forces an expansion of the stacked square-planar nickel units, resulting in an $\mathrm{Ni}_{\mathrm{sp}}-\mathrm{Ni}_{\mathrm{sp}}$ distance of $3.592(1) \AA$. The distances between these units and the pseudo-octahedral $\mathrm{Ni}^{\mathrm{II}}$ atom, however, remain similar $\left[\mathrm{Ni}_{\mathrm{sp}}-\mathrm{Ni}_{\text {oct }}=3.274(1)\right.$, 3.255 (1) $\AA$ ] . Overall, the entire structure retains all the other structural features that are present in the proton-bridged compound.

The phenanthrene backbones show pronounced twisting between their aromatic rings, which precludes conjugation across this unit. For the proton-bridged complex, the angle between mean planes within a single phenanthrene backbone ranges from $9.24(19)^{\circ}$ (between C45-C50 and C51C56) to $15.44(13)^{\circ}$ (between C59-C64 and C65-C70). For the 


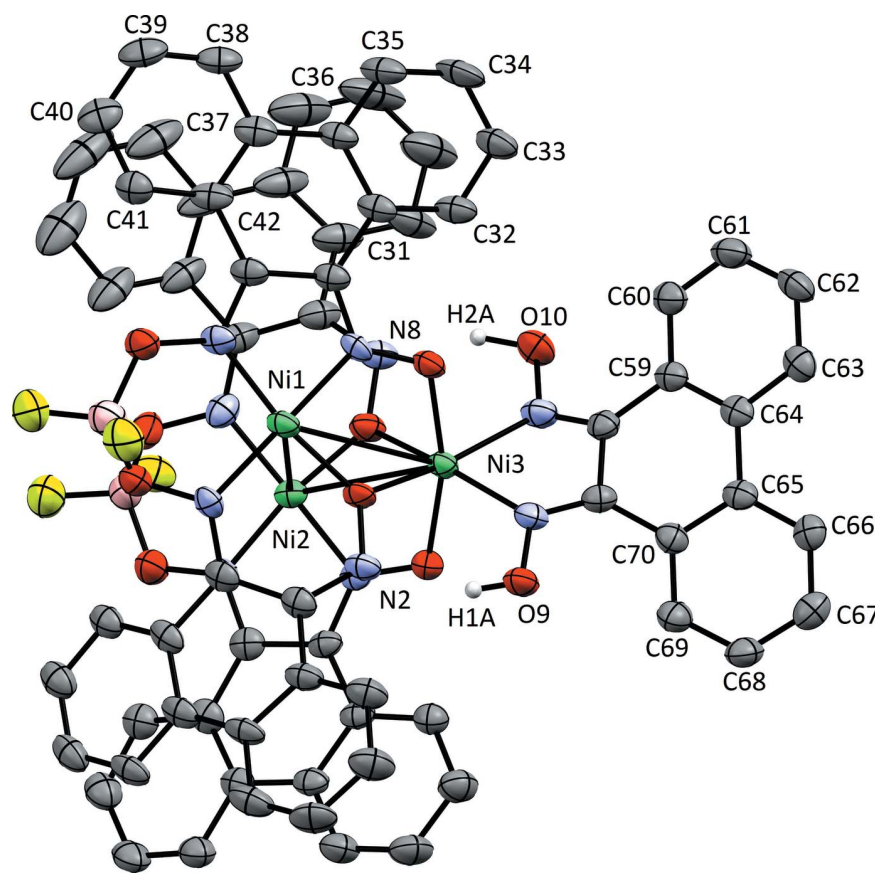

Figure 2

Displacement ellipsoid plot at the $50 \%$ probability level of $\left[\mathrm{Ni}_{3}\left(\mathrm{H}_{2}\right.\right.$ pqd $\left.)\left(\mathrm{BF}_{2} \mathrm{pqd}\right)_{2}(\mathrm{pqd})_{2}\right]$. $\mathrm{H}$ atoms (with the exception of hydrogen-bonded atoms) and solvent molecules have been omitted for clarity.

$\mathrm{BF}_{2}$-bridged complex, there is a wider range of angles, with $5.2(4)^{\circ}$ (between C31-C36 and C37-C42) being the smallest, and $17.5(3)^{\circ}$ (between C59-C64 and C65-C70) being the largest.
Table 1

Hydrogen-bond geometry $\left(\AA,^{\circ}\right)$ for (1).

\begin{tabular}{|c|c|c|c|c|}
\hline$D-\mathrm{H} \cdots A$ & $D-\mathrm{H}$ & $\mathrm{H} \cdots A$ & $D \cdots A$ & $D-\mathrm{H} \cdots A$ \\
\hline $\mathrm{O} 1-\mathrm{H} 1 A \cdots \mathrm{O} 5$ & $1.29(6)$ & $1.13(6)$ & $2.405(4)$ & $169(6)$ \\
\hline $\mathrm{O} 3-\mathrm{H} 2 A \cdots \mathrm{O} 7$ & $1.17(5)$ & $1.23(5)$ & $2.402(4)$ & $176(5)$ \\
\hline $\mathrm{O} 9-\mathrm{H} 3 A \cdots \mathrm{N} 4$ & $0.86(6)$ & $1.82(6)$ & $2.672(4)$ & $175(7)$ \\
\hline $\mathrm{O} 10-\mathrm{H} 4 A \cdots \mathrm{N} 6$ & $1.04(5)$ & $1.63(5)$ & $2.657(4)$ & $168(4)$ \\
\hline
\end{tabular}

Table 2

Hydrogen-bond geometry $\left(\AA,^{\circ}\right)$ for (2).

\begin{tabular}{lllll}
\hline$D-\mathrm{H} \cdots A$ & $D-\mathrm{H}$ & $\mathrm{H} \cdots A$ & $D \cdots A$ & $D-\mathrm{H} \cdots A$ \\
\hline $\mathrm{O} 9-\mathrm{H} 1 A \cdots \mathrm{N} 2$ & 0.85 & 1.96 & $2.771(6)$ & 158 \\
$\mathrm{O} 10-\mathrm{H} 2 A \cdots \mathrm{N} 8$ & 1.06 & 1.77 & $2.765(6)$ & 155 \\
\hline
\end{tabular}

\section{Supramolecular features}

The proton-bridged complex completes the macrocyclic coordination around the square-planar $\mathrm{Ni}^{\mathrm{II}}$ atoms by means of hydrogen bonds. Furthermore, the ligand that coordinates the pseudo-octahedral $\mathrm{Ni}^{\mathrm{II}}$ atom features hydrogen-bonding interactions (Table 1) between the oxime hydroxy groups and the ligands of the square-planar $\mathrm{Ni}^{\mathrm{II}}$ atoms. The nickel units show no direct interaction with their nearest neighbors in the extended lattice. Some $\pi$-stacking between adjacent molecules is, however, evident (Fig. 3). Two interactions were found, one with a centroid-centroid distance of 3.886 (2) $\AA$ (symmetry code: $\left.1-x,-\frac{1}{2}+y, \frac{3}{2}-z\right)$ and the other with a centroidcentroid distance of 4.256 (3) $\AA$ (symmetry code: $-x,-y$, $2-z$ ). In the latter case, although not aromatic, the distance to the centroid of the central ring of phenanthrene is shorter, with a distance of 3.528 (3) $\AA$. Toluene molecules occupy the solvent channels that are oriented along the $c$ axis.

Figure 3

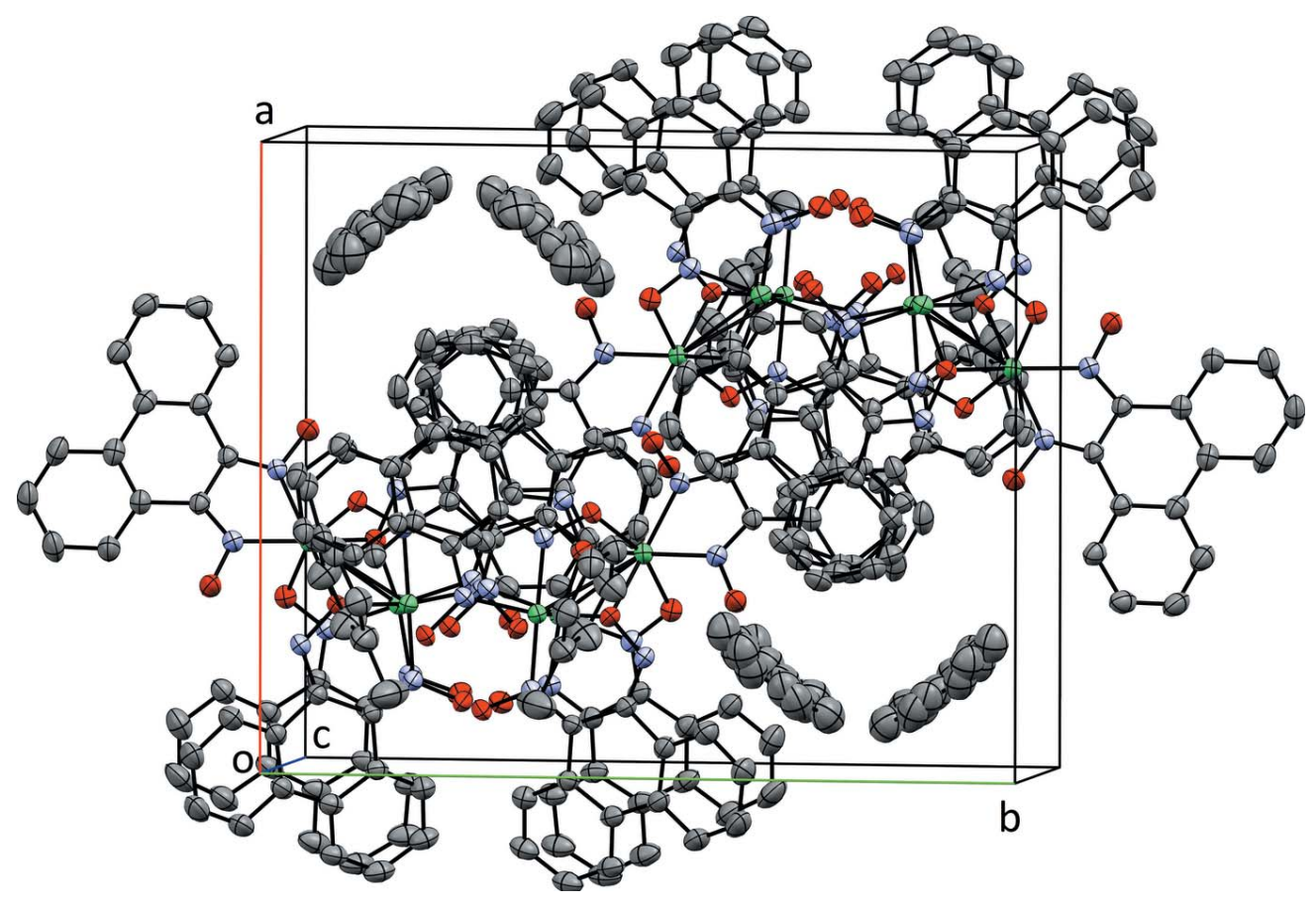

Packing diagram of $\left[\mathrm{Ni}_{3}\left(\mathrm{H}_{2} \mathrm{pqd}\right)(\mathrm{Hpqd})_{2}(\mathrm{pqd})_{2}\right]$, viewed approximately down the $c$-axis direction. 


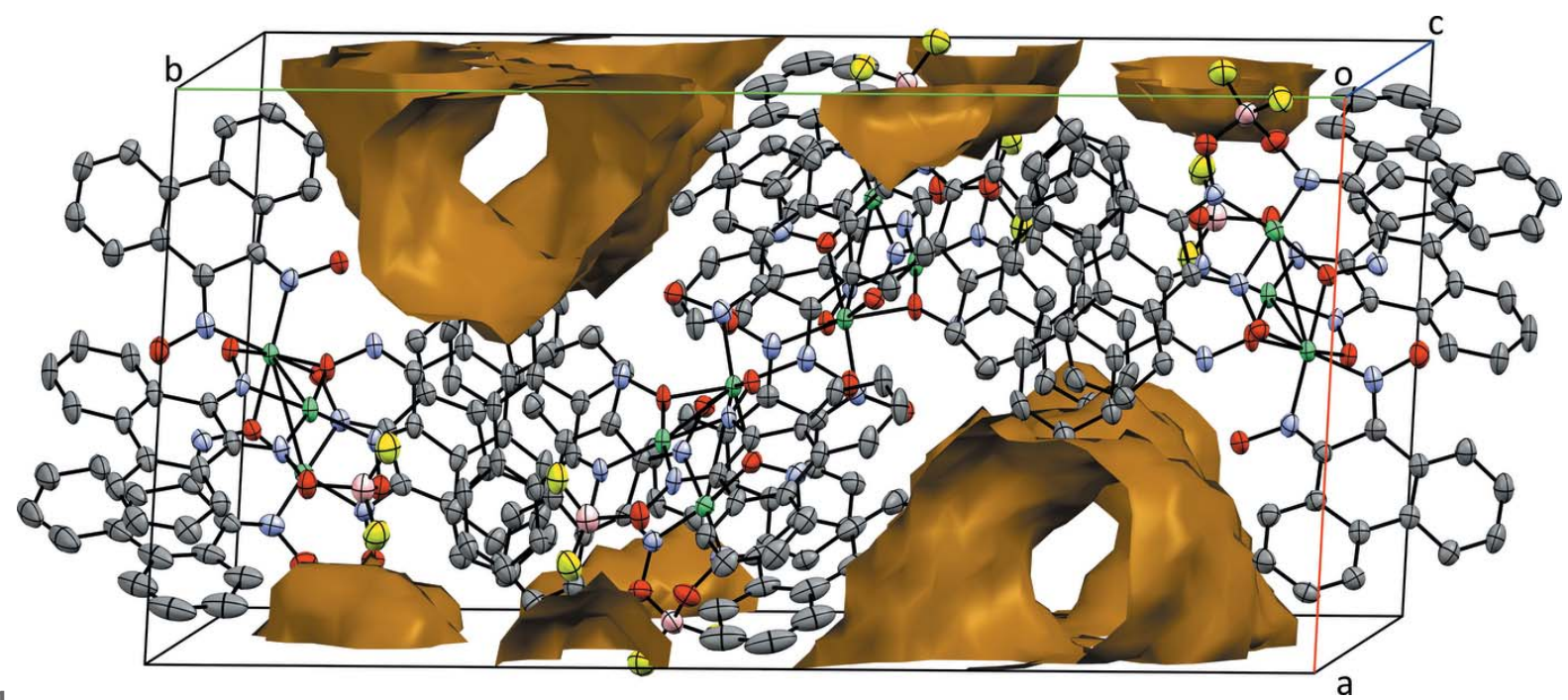

Figure 4

Packing diagram of $\mathrm{Ni}_{3}\left(\mathrm{H}_{2} \mathrm{pqd}\right)\left(\mathrm{BF}_{2} \mathrm{pqd}\right)_{2}(\mathrm{pqd})_{2}$, viewed approximately down the $c$-axis direction. Voids presented in brown were calculated in $M e r c u r y$ (Macrae et al., 2006) using a probe radius of $1.2 \AA$.

The $\mathrm{BF}_{2}$-bridged complex completes the macrocyclic coordination around the square-planar $\mathrm{Ni}^{\mathrm{II}}$ atoms by means of covalent $\mathrm{O}-\mathrm{B}-\mathrm{O}$ bonds. However, the hydrogen-bonding interactions (Table 2) that lock the pseudo-octahedral $\mathrm{Ni}^{\mathrm{II}}$ atom remain in place. The nickel units show no direct inter- action with their nearest neighbors in the extended lattice. A solvent channel oriented along the $c$ axis is also evident (Fig. 4). However, the extreme disorder of the solvent does not permit the determination of a suitable model.

Table 3

Experimental details.

(1)

Crystal data

Chemical formula

$M_{\mathrm{r}}$

Crystal system, space group

Temperature (K)

$a, b, c(\AA)$

$\beta\left({ }^{\circ}\right.$ )

$V\left(\AA^{3}\right)$

$Z$

Radiation type

$\mu\left(\mathrm{mm}^{-1}\right)$

Crystal size (mm)

Data collection

Diffractometer

Absorption correction

$T_{\min }, T_{\max }$

No. of measured, independent and observed

$[I>2 \sigma(I)]$ reflections

$R_{\text {int }}$

$(\sin \theta / \lambda)_{\max }\left(\AA^{-1}\right)$

Refinement

$R\left[F^{2}>2 \sigma\left(F^{2}\right)\right], w R\left(F^{2}\right), S$

No. of reflections

No. of parameters

No. of restraints

$\mathrm{H}$-atom treatment

$\Delta \rho_{\max }, \Delta \rho_{\min }\left(\mathrm{e} \AA^{-3}\right)$
$\left[\mathrm{Ni}_{3}\left(\mathrm{C}_{14} \mathrm{H}_{8} \mathrm{~N}_{2} \mathrm{O}_{2}\right)_{2}\left(\mathrm{C}_{14} \mathrm{H}_{9} \mathrm{~N}_{2} \mathrm{O}_{2}\right)_{2}\right.$ -

$\left.\left(\mathrm{C}_{14} \mathrm{H}_{10} \mathrm{~N}_{2} \mathrm{O}_{2}\right)\right] \cdot 2 \mathrm{C}_{7} \mathrm{H}_{8}$

1545.55

Monoclinic, $P 2_{1} / c$

100

15.973 (3), 18.639 (3), 22.785 (4)

$101.757(4)$

$6641.1(19)$

4

Mo $K \alpha$

0.92

$0.16 \times 0.11 \times 0.11$

Rigaku Saturn724+

Multi-scan (ABSCOR; Higashi, 1995)

$0.799,1.000$

79592, 15260, 13993

0.054

0.650

$0.067,0.151,1.21$

15260

1046

291

$\mathrm{H}$ atoms treated by a mixture of independent and constrained refinement

$0.56,-0.68$
(2)

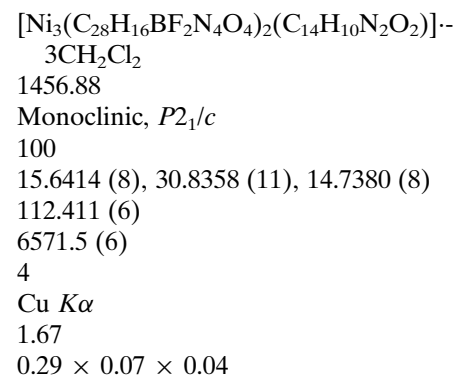

Agilent SuperNova Dual Source diffractometer with an Atlas detector

Multi-scan (CrysAlis PRO; Agilent, 2014)

$0.303,1.000$

$25337,13041,8782$

0.060

0.626

$0.077,0.226,1.03$

13041

896

0

$\mathrm{H}$ atoms treated by a mixture of independent and constrained refinement

$1.16,-0.83$

Computer programs: CrystalClear (Rigaku Inc, 2008), CrysAlis PRO (Agilent, 2014), SIR2004 (Burla et al., 2005), OLEX2 (Dolomanov et al., 2009), SHELXL2013 (Sheldrick, 2015), Mercury (Macrae et al., 2006), publCIF (Westrip, 2010) and WinGX (Farrugia, 1999). 


\section{Synthesis and crystallization}

The parent ligand, $\mathrm{pqdH}_{2}(0.75 \mathrm{~g} ; 3.1 \mathrm{mmol})$, was dissolved in $100 \mathrm{ml}$ of ethanol, to which nickel(II) acetate $(0.33 \mathrm{~g}$, $1.3 \mathrm{mmol}$ ) was added. A red precipitate began to form after approximately $30 \mathrm{~min}$. The solution was then allowed to stir for $1 \mathrm{~h}$, followed by cooling in a freezer and filtration of the crude product (yield: $272 \mathrm{mg}, 0.2 \mathrm{mmol}, 32 \%$ ). The resulting product was dissolved in DMF solution and layered with toluene, resulting in the formation of crystals of $\left[\mathrm{Ni}_{3}\left(\mathrm{H}_{2} \mathrm{pqd}\right)(\mathrm{Hpqd})_{2}(\mathrm{pqd})_{2}\right]$ after a period of $3-4 \mathrm{~d}$. The crystals grew as red blocks with an asymmetric unit consisting of a complete $\left[\mathrm{Ni}_{3}\left(\mathrm{H}_{2} \mathrm{pqd}\right)(\mathrm{Hpqd})_{2}(\mathrm{pqd})_{2}\right]$ molecule and two toluene solvent molecules.

The foregoing complex is stable enough to undergo a fluoridoboration reaction with boron trifluoride, thereby affording the compound $\left[\mathrm{Ni}_{3}\left(\mathrm{H}_{2} \mathrm{pqd}\right)\left(\mathrm{BF} \mathrm{F}_{2} \mathrm{pqd}\right)_{2}(\mathrm{pqd})_{2}\right]$. $\left[\mathrm{Ni}_{3}\left(\mathrm{H}_{2} \mathrm{pqd}\right)(\mathrm{Hpqd})_{2}(\mathrm{pqd})_{2}\right]$ was diluted in diethyl ether, thereby creating a slurry. One $\mathrm{ml}$ of 1.0 molar $\mathrm{BF}_{3}-\mathrm{OEt}_{2}$ (in ether) was then added and the mixture was allowed to react overnight. The resulting precipitate was then filtered off and washed thoroughly with $\mathrm{EtOH}$ and $\mathrm{Et}_{2} \mathrm{O}$. The resulting precipitate was then dissolved in dichloromethane (DCM) and filtered through Celite (yield: $43 \mathrm{mg}, 30 \mu \mathrm{mol}, 79 \%$ ). Subsequently, a crop of red block-shaped crystals was grown by solvent evaporation over a period of one day.

\section{Refinement}

Crystal data, data collection and structure refinement details are summarized in Table 3. In proton-bridged structure (1), atoms $\mathrm{H} 1 A, \mathrm{H} 2 A$ and $\mathrm{H} 4 A$ were found by assignment of difference map peaks and refined isotropically without geometrical constraints. The proton $\mathrm{H} 3 A$ was initially placed with the SHELXL HFIX 147 command (refinement on rotation) on O9, but was refined freely. Four distinct hydrogenbonding interactions were evident in the trinuclear cluster. Finally, there were two $\mathrm{O}-\mathrm{H}-\mathrm{O}$ interactions between an oxime and oximato of each $[\mathrm{Ni}(\mathrm{Hpqd})(\mathrm{pqd})]^{-}$unit that could not be resolved due to rapid conversion to $[\mathrm{Ni}(\mathrm{pqd})(\mathrm{Hpqd})]^{-}$.
All the restraints that are reported were included for the modelling of the disordered toluene solvent molecules.

In the case of $\mathrm{BF}_{2}$-bridged structure (2), atoms $\mathrm{H} 1 A$ and $\mathrm{H} 2 A$ were affixed to $\mathrm{O} 9$ and $\mathrm{O} 10$, respectively. They were then refined isotropically without rotational constraints. The SQUEEZE routine (Spek, 2015) as implemented in PLATON (Spek, 2009) was used to remove the electron density of three solvent DCM molecules per unit cell (calculated: $134 \mathrm{e}^{-}$; $593 \AA^{3}$ ).

\section{Acknowledgements}

The data were collected using instrumentation purchased with funds provided by the National Science Foundation (grant No. 0741973). The chemistry was supported by the Robert A. Welch Foundation (grant F-0003).

\section{References}

Agilent (2014). CrysAlis PRO. Agilent Technologies Ltd, Yarnton, England.

Aromí, G. \& Brechin, E. K. (2006). Single-Molecule Magnets and Related Phenomena, edited by R. Winpenny, pp. 1-67. Berlin, Heidelberg: Springer.

Biswas, B., Pieper, U., Weyhermüller, T. \& Chaudhuri, P. (2009). Inorg. Chem. 48, 6781-6793.

Burla, M. C., Caliandro, R., Camalli, M., Carrozzini, B., Cascarano, G. L., De Caro, L., Giacovazzo, C., Polidori, G. \& Spagna, R. (2005). J. Appl. Cryst. 38, 381-388.

Chaudhuri, P. (2003). Coord. Chem. Rev. 243, 143-190.

Dolomanov, O. V., Bourhis, L. J., Gildea, R. J., Howard, J. A. K. \& Puschmann, H. (2009). J. Appl. Cryst. 42, 339-341.

Farrugia, L. J. (1999). J. Appl. Cryst. 32, 837-838.

Higashi, T. (1995). ABSCOR. Rigaku Corporation, Tokyo, Japan.

Jiang, Y.-B., Kou, H.-Z., Wang, R.-J., Cui, A.-L. \& Ribas, J. (2005). Inorg. Chem. 44, 709-715.

Macrae, C. F., Edgington, P. R., McCabe, P., Pidcock, E., Shields, G. P., Taylor, R., Towler, M. \& van de Streek, J. (2006). J. Appl. Cryst. 39, 453-457.

Rigaku Inc. (2008). CrystalClear. Rigaku Corporation, Tokyo, Japan. Schmidt, J. \& Söll, J. (1907). Ber. Dtsch. Chem. Ges. 40, 2454-2460.

Sheldrick, G. M. (2015). Acta Cryst. C71, 3-8.

Spek, A. L. (2009). Acta Cryst. D65, 148-155.

Spek, A. L. (2015). Acta Cryst. C71, 9-18.

Westrip, S. P. (2010). J. Appl. Cryst. 43, 920-925. 


\section{supporting information}

Acta Cryst. (2016). E72, 538-542 [https://doi.org/10.1107/S2056989016004023]

\section{Trinuclear nickel coordination complexes of phenanthrene-9,10-dione dioxime}

\section{Owen M. Williams and Alan H. Cowley}

Computing details

Data collection: CrystalClear (Rigaku Inc, 2008) for (1); CrysAlis PRO (Agilent, 2014) for (2). Cell refinement:

CrystalClear (Rigaku Inc, 2008) for (1); CrysAlis PRO (Agilent, 2014) for (2). Data reduction: CrystalClear (Rigaku Inc, 2008) for (1); CrysAlis PRO (Agilent, 2014) for (2). Program(s) used to solve structure: SIR2004 (Burla et al., 2005) for (1); OLEX2 (Dolomanov et al., 2009) for (2). For both compounds, program(s) used to refine structure: SHELXL2013 (Sheldrick, 2015); molecular graphics: Mercury (Macrae et al., 2006); software used to prepare material for publication: publCIF (Westrip, 2010) and WinGX (Farrugia, 1999).

(1) $\operatorname{Bis}\left[\mu_{2}-9,10-b i s(o x i d o i m i n o) p h e n a n t h r e n e\right] b i s\left[\mu_{2}-10\right.$-(oxidoimino) phenanthrene-9-one oxime] (phenanthrene-9,10-dione dioxime)trinickel(II) toluene disolvate

Crystal data

$\left[\mathrm{Ni}_{3}\left(\mathrm{C}_{14} \mathrm{H}_{8} \mathrm{~N}_{2} \mathrm{O}_{2}\right)_{2}\left(\mathrm{C}_{14} \mathrm{H}_{9} \mathrm{~N}_{2} \mathrm{O}_{2}\right)_{2}\left(\mathrm{C}_{14} \mathrm{H}_{10} \mathrm{~N}_{2} \mathrm{O}_{2}\right)\right] \cdot 2 \mathrm{C}_{7} \mathrm{H}_{8}$ $M_{r}=1545.55$

Monoclinic, $P 2_{1} / c$

$a=15.973(3) \AA$

$b=18.639$ (3) $\AA$

$c=22.785(4) \AA$

$\beta=101.757(4)^{\circ}$

$V=6641.1(19) \AA^{3}$

$Z=4$

\section{Data collection}

Rigaku Saturn724+ (2x2 bin mode) diffractometer

Radiation source: Sealed Tube

Detector resolution: 28.5714 pixels $\mathrm{mm}^{-1}$

dtprofit.ref scans

Absorption correction: multi-scan

(ABSCOR; Higashi, 1995)

$T_{\min }=0.799, T_{\max }=1.000$

Refinement

Refinement on $F^{2}$

Least-squares matrix: full

$R\left[F^{2}>2 \sigma\left(F^{2}\right)\right]=0.067$

$w R\left(F^{2}\right)=0.151$

$S=1.21$

15260 reflections

1046 parameters

291 restraints
$F(000)=3192$

$D_{\mathrm{x}}=1.546 \mathrm{Mg} \mathrm{m}^{-3}$

Mo $K \alpha$ radiation, $\lambda=0.71069 \AA$

Cell parameters from 20424 reflections

$\theta=1.3-31.1^{\circ}$

$\mu=0.92 \mathrm{~mm}^{-1}$

$T=100 \mathrm{~K}$

Block, red

$0.16 \times 0.11 \times 0.11 \mathrm{~mm}$

79592 measured reflections

15260 independent reflections

13993 reflections with $I>2 \sigma(I)$

$R_{\text {int }}=0.054$

$\theta_{\max }=27.5^{\circ}, \theta_{\min }=1.8^{\circ}$

$h=-20 \rightarrow 20$

$k=-24 \rightarrow 24$

$l=-29 \rightarrow 29$

Hydrogen site location: mixed

$\mathrm{H}$ atoms treated by a mixture of independent and constrained refinement

$w=1 /\left[\sigma^{2}\left(F_{0}^{2}\right)+(0.0495 P)^{2}+7.4732 P\right]$

where $P=\left(F_{\mathrm{o}}^{2}+2 F_{\mathrm{c}}^{2}\right) / 3$

$(\Delta / \sigma)_{\max }=0.001$

$\Delta \rho_{\max }=0.56 \mathrm{e}^{-3}$

$\Delta \rho_{\min }=-0.68 \mathrm{e} \AA^{-3}$ 


\section{Special details}

Geometry. All esds (except the esd in the dihedral angle between two 1.s. planes) are estimated using the full covariance matrix. The cell esds are taken into account individually in the estimation of esds in distances, angles and torsion angles; correlations between esds in cell parameters are only used when they are defined by crystal symmetry. An approximate (isotropic) treatment of cell esds is used for estimating esds involving l.s. planes.

Fractional atomic coordinates and isotropic or equivalent isotropic displacement parameters $\left(\AA^{2}\right)$

\begin{tabular}{|c|c|c|c|c|c|}
\hline & $x$ & $y$ & $z$ & $U_{\text {iso }} * / U_{\text {eq }}$ & Occ. $(<1)$ \\
\hline $\mathrm{C} 1$ & $0.3573(2)$ & $0.26380(17)$ & $0.77353(15)$ & $0.0300(7)$ & \\
\hline $\mathrm{C} 2$ & $0.4143(2)$ & $0.20289(17)$ & $0.79218(14)$ & $0.0287(6)$ & \\
\hline $\mathrm{C} 3$ & $0.5058(2)$ & $0.21463(17)$ & $0.81436(14)$ & $0.0289(7)$ & \\
\hline $\mathrm{C} 4$ & $0.5622(2)$ & $0.15956(19)$ & $0.83987(15)$ & $0.0341(7)$ & \\
\hline $\mathrm{H} 4$ & 0.5433 & 0.1123 & 0.8376 & $0.041^{*}$ & \\
\hline $\mathrm{C} 5$ & $0.6447(2)$ & $0.1749(2)$ & $0.86803(16)$ & $0.0392(8)$ & \\
\hline H5 & 0.6811 & 0.1381 & 0.8850 & $0.047^{*}$ & \\
\hline C6 & $0.6738(2)$ & $0.2450(2)$ & 0.87123 & $0.0423(9)$ & \\
\hline H6 & 0.7292 & 0.2554 & 0.8913 & $0.051^{*}$ & \\
\hline $\mathrm{C} 7$ & $0.6209(2)$ & $0.2991(2)$ & $0.84477(17)$ & $0.0412(8)$ & \\
\hline $\mathrm{H} 7$ & 0.6418 & 0.3458 & 0.8465 & $0.049^{*}$ & \\
\hline $\mathrm{C} 8$ & $0.5364(2)$ & $0.28614(18)$ & $0.81521(15)$ & $0.0318(7)$ & \\
\hline C9 & 0.4809 (2) & $0.34500(17)$ & $0.78633(15)$ & $0.0306(7)$ & \\
\hline $\mathrm{C} 10$ & $0.5151(2)$ & $0.41244(19)$ & $0.77676(16)$ & $0.0371(8)$ & \\
\hline $\mathrm{H} 10$ & 0.5735 & 0.4200 & 0.7897 & $0.045^{*}$ & \\
\hline C11 & $0.4652(2)$ & 0.46755 (19) & $0.74890(17)$ & $0.0403(8)$ & \\
\hline H11 & 0.4897 & 0.5119 & 0.7442 & $0.048^{*}$ & \\
\hline $\mathrm{C} 12$ & $0.3783(2)$ & $0.45710(18)$ & $0.72779(17)$ & $0.0388(8)$ & \\
\hline H12 & 0.3445 & 0.4941 & 0.7085 & $0.047^{*}$ & \\
\hline $\mathrm{C} 13$ & $0.3423(2)$ & $0.39116(17)$ & $0.73569(16)$ & $0.0334(7)$ & \\
\hline H13 & 0.2841 & 0.3841 & 0.7212 & $0.040^{*}$ & \\
\hline $\mathrm{C} 14$ & $0.3922(2)$ & $0.33465(16)$ & $0.76530(15)$ & $0.0294(7)$ & \\
\hline $\mathrm{C} 15$ & $0.3654(2)$ & $0.24577(17)$ & $0.93609(14)$ & $0.0292(7)$ & \\
\hline $\mathrm{C} 16$ & $0.4342(2)$ & $0.19229(17)$ & $0.94884(14)$ & $0.0283(6)$ & \\
\hline $\mathrm{C} 17$ & $0.5203(2)$ & $0.21590(17)$ & $0.98016(14)$ & $0.0293(7)$ & \\
\hline $\mathrm{C} 18$ & $0.5819(2)$ & $0.16511(18)$ & $1.00548(14)$ & $0.0327(7)$ & \\
\hline H18 & 0.5684 & 0.1165 & 1.0024 & $0.039^{*}$ & \\
\hline $\mathrm{C} 19$ & $0.6622(2)$ & $0.1864(2)$ & $1.03489(15)$ & $0.0364(8)$ & \\
\hline H19 & 0.7032 & 0.1524 & 1.0507 & $0.044^{*}$ & \\
\hline $\mathrm{C} 20$ & $0.6815(2)$ & $0.2591(2)$ & $1.04065(17)$ & $0.0410(8)$ & \\
\hline $\mathrm{H} 20$ & 0.7354 & 0.2738 & 1.0606 & $0.049^{*}$ & \\
\hline $\mathrm{C} 21$ & $0.6211(2)$ & $0.3092(2)$ & $1.01689(16)$ & $0.0396(8)$ & \\
\hline $\mathrm{H} 21$ & 0.6345 & 0.3577 & 1.0218 & $0.048^{*}$ & \\
\hline $\mathrm{C} 22$ & $0.5392(2)$ & $0.28906(18)$ & $0.98522(14)$ & $0.0317(7)$ & \\
\hline $\mathrm{C} 23$ & $0.4766(2)$ & $0.34208(17)$ & $0.95560(15)$ & $0.0317(7)$ & \\
\hline $\mathrm{C} 24$ & $0.4995(2)$ & $0.41366(19)$ & $0.94928(17)$ & $0.0386(8)$ & \\
\hline $\mathrm{H} 24$ & 0.5548 & 0.4282 & 0.9663 & $0.046^{*}$ & \\
\hline $\mathrm{C} 25$ & $0.4432(3)$ & $0.4633(2)$ & $0.91884(18)$ & $0.0428(9)$ & \\
\hline $\mathrm{H} 25$ & 0.4599 & 0.5108 & 0.9166 & $0.051^{*}$ & \\
\hline
\end{tabular}




\begin{tabular}{|c|c|c|c|c|}
\hline $\mathrm{C} 26$ & $0.3618(2)$ & $0.44223(19)$ & $0.89172(17)$ & $0.0408(8)$ \\
\hline $\mathrm{H} 26$ & 0.3242 & 0.4751 & 0.8696 & $0.049^{*}$ \\
\hline $\mathrm{C} 27$ & $0.3360(2)$ & $0.37214(18)$ & $0.89741(16)$ & $0.0363(8)$ \\
\hline $\mathrm{H} 27$ & 0.2809 & 0.3585 & 0.8790 & $0.044 *$ \\
\hline $\mathrm{C} 28$ & $0.3911(2)$ & $0.32129(17)$ & $0.93032(15)$ & $0.0312(7)$ \\
\hline $\mathrm{C} 29$ & $0.0764(2)$ & $0.10264(17)$ & $0.76379(14)$ & $0.0291(7)$ \\
\hline $\mathrm{C} 30$ & $0.1064(2)$ & $0.03055(17)$ & $0.75287(15)$ & $0.0309(7)$ \\
\hline $\mathrm{C} 31$ & $0.0435(2)$ & $-0.02258(18)$ & $0.72335(15)$ & $0.0346(7)$ \\
\hline C32 & $0.0685(2)$ & $-0.0803(2)$ & $0.69200(18)$ & $0.0429(9)$ \\
\hline H32 & 0.1254 & -0.0851 & 0.6889 & $0.051^{*}$ \\
\hline $\mathrm{C} 33$ & $0.0088(3)$ & $-0.1305(2)$ & $0.6655(2)$ & $0.0529(10)$ \\
\hline H33 & 0.0255 & -0.1689 & 0.6445 & $0.064^{*}$ \\
\hline $\mathrm{C} 34$ & $-0.0749(3)$ & $-0.1236(2)$ & $0.6701(2)$ & $0.0539(11)$ \\
\hline H34 & -0.1146 & -0.1579 & 0.6526 & $0.065^{*}$ \\
\hline $\mathrm{C} 35$ & $-0.1009(2)$ & $-0.0668(2)$ & $0.70016(19)$ & $0.0472(9)$ \\
\hline H35 & -0.1581 & -0.0628 & 0.7026 & $0.057^{*}$ \\
\hline $\mathrm{C} 36$ & $-0.0422(2)$ & $-0.01423(19)$ & $0.72739(16)$ & $0.0366(8)$ \\
\hline $\mathrm{C} 37$ & $-0.0676(2)$ & $0.0483(2)$ & $0.75883(16)$ & $0.0360(8)$ \\
\hline $\mathrm{C} 38$ & $-0.1479(2)$ & $0.0511(2)$ & $0.77467(18)$ & $0.0434(9)$ \\
\hline H38 & -0.1845 & 0.0120 & 0.7661 & $0.052^{*}$ \\
\hline C39 & $-0.1741(2)$ & $0.1100(2)$ & $0.80252(18)$ & $0.0462(9)$ \\
\hline H39 & -0.2275 & 0.1102 & 0.8127 & $0.055^{*}$ \\
\hline $\mathrm{C} 40$ & $-0.1214(2)$ & $0.1683(2)$ & $0.81519(18)$ & $0.0450(9)$ \\
\hline $\mathrm{H} 40$ & -0.1397 & 0.2086 & 0.8331 & $0.054 *$ \\
\hline $\mathrm{C} 41$ & $-0.0410(2)$ & $0.1676(2)$ & $0.80142(17)$ & $0.0393(8)$ \\
\hline H41 & -0.0054 & 0.2072 & 0.8109 & $0.047^{*}$ \\
\hline $\mathrm{C} 42$ & $-0.0122(2)$ & $0.10822(18)$ & $0.77336(15)$ & $0.0323(7)$ \\
\hline $\mathrm{C} 43$ & $0.0836(2)$ & $0.08681(19)$ & $0.92655(15)$ & $0.0345(7)$ \\
\hline $\mathrm{C} 44$ & $0.1243(2)$ & $0.02254(19)$ & $0.90908(15)$ & $0.0346(7)$ \\
\hline $\mathrm{C} 45$ & $0.0739(2)$ & $-0.04203(19)$ & $0.89031(16)$ & $0.0378(8)$ \\
\hline $\mathrm{C} 46$ & $0.1065(3)$ & $-0.1008(2)$ & $0.86405(17)$ & $0.0433(9)$ \\
\hline H46 & 0.1639 & -0.1016 & 0.8616 & $0.052^{*}$ \\
\hline $\mathrm{C} 47$ & $0.0542(3)$ & $-0.1577(2)$ & $0.8417(2)$ & $0.0555(11)$ \\
\hline H47 & 0.0760 & -0.1960 & 0.8233 & $0.067 *$ \\
\hline $\mathrm{C} 48$ & $-0.0306(3)$ & $-0.1576(3)$ & $0.8468(2)$ & $0.0633(13)$ \\
\hline $\mathrm{H} 48$ & -0.0655 & -0.1963 & 0.8323 & $0.076^{*}$ \\
\hline $\mathrm{C} 49$ & $-0.0636(3)$ & $-0.1003(2)$ & $0.8731(2)$ & $0.0535(11)$ \\
\hline H49 & -0.1209 & -0.1011 & 0.8758 & $0.064 *$ \\
\hline $\mathrm{C} 50$ & $-0.0134(2)$ & $-0.0412(2)$ & $0.89578(16)$ & $0.0424(9)$ \\
\hline $\mathrm{C} 51$ & $-0.0494(2)$ & $0.0194(2)$ & $0.92335(16)$ & $0.0408(9)$ \\
\hline $\mathrm{C} 52$ & $-0.1321(3)$ & $0.0157(2)$ & $0.93653(19)$ & $0.0505(10)$ \\
\hline H52 & -0.1643 & -0.0258 & 0.9266 & $0.061^{*}$ \\
\hline C53 & $-0.1662(2)$ & $0.0706(3)$ & $0.96312(19)$ & $0.0524(11)$ \\
\hline H53 & -0.2208 & 0.0661 & 0.9712 & $0.063^{*}$ \\
\hline C54 & $-0.1207(2)$ & $0.1331(3)$ & $0.97825(18)$ & $0.0507(10)$ \\
\hline H54 & -0.1443 & 0.1706 & 0.9964 & $0.061^{*}$ \\
\hline C55 & $-0.0392(2)$ & $0.1395(2)$ & $0.96614(17)$ & $0.0430(9)$ \\
\hline H55 & -0.0084 & 0.1816 & 0.9762 & $0.052 *$ \\
\hline
\end{tabular}




\begin{tabular}{|c|c|c|c|c|c|}
\hline C56 & $-0.0028(2)$ & $0.0833(2)$ & $0.93893(15)$ & $0.0379(8)$ & \\
\hline C57 & $0.4778(2)$ & $-0.09599(17)$ & $0.87975(15)$ & $0.0311(7)$ & \\
\hline C58 & $0.4046(2)$ & $-0.13101(17)$ & $0.83829(15)$ & $0.0305(7)$ & \\
\hline C59 & $0.4055(2)$ & $-0.20914(17)$ & $0.83084(14)$ & $0.0320(7)$ & \\
\hline C60 & $0.3314(2)$ & -0.24949 (19) & $0.80879(17)$ & $0.0412(8)$ & \\
\hline H60 & 0.2797 & -0.2260 & 0.7955 & $0.049^{*}$ & \\
\hline C61 & $0.3342(3)$ & $-0.3230(2)$ & $0.80656(19)$ & $0.0508(10)$ & \\
\hline H61 & 0.2842 & -0.3488 & 0.7931 & $0.061^{*}$ & \\
\hline C62 & $0.4101(3)$ & $-0.3585(2)$ & $0.82407(19)$ & $0.0534(11)$ & \\
\hline H62 & 0.4120 & -0.4082 & 0.8214 & $0.064 *$ & \\
\hline C63 & $0.4839(3)$ & $-0.3203(2)$ & 0.84575 (19) & $0.0487(10)$ & \\
\hline H63 & 0.5352 & -0.3449 & 0.8576 & $0.058^{*}$ & \\
\hline C64 & $0.4835(2)$ & $-0.24541(18)$ & $0.85028(16)$ & $0.0367(8)$ & \\
\hline C65 & $0.5628(2)$ & $-0.20611(19)$ & $0.87678(16)$ & $0.0367(8)$ & \\
\hline C66 & $0.6421(2)$ & $-0.2409(2)$ & $0.88592(18)$ & $0.0453(9)$ & \\
\hline H66 & 0.6448 & -0.2878 & 0.8724 & $0.054 *$ & \\
\hline C67 & $0.7154(3)$ & $-0.2078(2)$ & $0.91426(19)$ & $0.0483(10)$ & \\
\hline H67 & 0.7673 & -0.2321 & 0.9199 & $0.058^{*}$ & \\
\hline C68 & $0.7123(2)$ & $-0.1384(2)$ & $0.93455(18)$ & $0.0441(9)$ & \\
\hline H68 & 0.7622 & -0.1167 & 0.9550 & $0.053^{*}$ & \\
\hline C69 & $0.6367(2)$ & -0.10049 (19) & $0.92512(16)$ & $0.0372(8)$ & \\
\hline H69 & 0.6360 & -0.0534 & 0.9384 & $0.045^{*}$ & \\
\hline $\mathrm{C} 70$ & $0.5598(2)$ & $-0.13368(18)$ & $0.89503(15)$ & $0.0321(7)$ & \\
\hline $\mathrm{C} 71$ & $0.1134(3)$ & $0.1368(4)$ & $0.5502(3)$ & $0.100(2)$ & \\
\hline H71A & 0.0755 & 0.1011 & 0.5291 & $0.150^{*}$ & \\
\hline H71B & 0.1188 & 0.1755 & 0.5235 & $0.150^{*}$ & \\
\hline $\mathrm{H} 71 \mathrm{C}$ & 0.0906 & 0.1547 & 0.5832 & $0.150^{*}$ & \\
\hline $\mathrm{C} 72$ & $0.2006(3)$ & $0.1037(3)$ & $0.5736(2)$ & $0.0596(12)$ & \\
\hline $\mathrm{C} 73$ & $0.2598(3)$ & $0.0972(2)$ & $0.5371(2)$ & $0.0513(10)$ & \\
\hline H73 & 0.2466 & 0.1145 & 0.4980 & $0.062 *$ & \\
\hline $\mathrm{C} 74$ & $0.3375(3)$ & $0.0656(2)$ & $0.55792(19)$ & $0.0501(10)$ & \\
\hline $\mathrm{H} 74$ & 0.3767 & 0.0618 & 0.5330 & $0.060^{*}$ & \\
\hline $\mathrm{C} 75$ & $0.3582(3)$ & $0.0391(2)$ & 0.61574 (19) & $0.0502(10)$ & \\
\hline $\mathrm{H} 75$ & 0.4106 & 0.0167 & 0.6294 & $0.060^{*}$ & \\
\hline $\mathrm{C} 76$ & $0.3013(3)$ & $0.0460(3)$ & $0.6527(2)$ & $0.0634(13)$ & \\
\hline $\mathrm{H} 76$ & 0.3150 & 0.0287 & 0.6917 & $0.076^{*}$ & \\
\hline $\mathrm{C} 77$ & $0.2235(3)$ & $0.0787(3)$ & $0.6320(2)$ & $0.0722(15)$ & \\
\hline $\mathrm{H} 77$ & 0.1857 & 0.0842 & 0.6578 & $0.087^{*}$ & \\
\hline $\mathrm{C} 78$ & $-0.1860(5)$ & $0.0902(4)$ & $0.4357(4)$ & $0.087(2)$ & 0.66 \\
\hline H78A & -0.1443 & 0.0585 & 0.4248 & $0.131^{*}$ & 0.66 \\
\hline H78B & -0.1928 & 0.1314 & 0.4099 & $0.131^{*}$ & 0.66 \\
\hline $\mathrm{H} 78 \mathrm{C}$ & -0.2397 & 0.0656 & 0.4315 & $0.131^{*}$ & 0.66 \\
\hline $\mathrm{C} 79$ & $-0.1572(3)$ & $0.1133(3)$ & $0.4982(3)$ & $0.0727(19)$ & 0.66 \\
\hline $\mathrm{C} 80$ & $-0.1792(5)$ & $0.0748(4)$ & $0.5449(3)$ & $0.080(2)$ & 0.66 \\
\hline $\mathrm{H} 80$ & -0.2124 & 0.0336 & 0.5364 & $0.096^{*}$ & 0.66 \\
\hline $\mathrm{C} 81$ & $-0.1524(6)$ & $0.0964(5)$ & $0.6039(4)$ & $0.086(3)$ & 0.66 \\
\hline $\mathrm{H} 81$ & -0.1681 & 0.0703 & 0.6348 & $0.103^{*}$ & 0.66 \\
\hline $\mathrm{C} 82$ & $-0.1021(6)$ & $0.1574(5)$ & $0.6167(3)$ & $0.082(3)$ & 0.66 \\
\hline
\end{tabular}




\begin{tabular}{|c|c|c|c|c|c|}
\hline H82 & -0.0833 & 0.1721 & 0.6562 & $0.098 *$ & 0.66 \\
\hline C83 & $-0.0800(5)$ & $0.1963(4)$ & $0.5703(3)$ & $0.083(2)$ & 0.66 \\
\hline H83 & -0.0466 & 0.2373 & 0.5789 & $0.100 *$ & 0.66 \\
\hline C84 & $-0.1068(4)$ & 0.1750 & $0.5113(3)$ & 0.0769 (19) & 0.66 \\
\hline H84 & -0.0916 & 0.2015 & 0.4805 & $0.092 *$ & 0.66 \\
\hline C78A & $-0.1035(9)$ & $0.1587(8)$ & $0.4495(6)$ & $0.078(4)$ & 0.34 \\
\hline H78D & -0.0889 & 0.2087 & 0.4523 & $0.118^{*}$ & 0.34 \\
\hline H78E & -0.1505 & 0.1513 & 0.4163 & $0.118 *$ & 0.34 \\
\hline $\mathrm{H} 78 \mathrm{~F}$ & -0.0551 & 0.1314 & 0.4433 & $0.118^{*}$ & 0.34 \\
\hline C79A & $-0.1290(7)$ & $0.1344(6)$ & $0.5073(7)$ & $0.080(3)$ & 0.34 \\
\hline C81A & $-0.2097(10)$ & $0.0579(9)$ & $0.5603(8)$ & $0.082(3)$ & 0.34 \\
\hline H81A & -0.2488 & 0.0208 & 0.5596 & $0.098^{*}$ & 0.34 \\
\hline C80A & $-0.1873(8)$ & $0.0791(7)$ & $0.5038(8)$ & $0.079(3)$ & 0.34 \\
\hline $\mathrm{H} 80 \mathrm{~A}$ & -0.2108 & 0.0567 & 0.4677 & $0.094^{*}$ & 0.34 \\
\hline C82A & $-0.1790(13)$ & $0.0872(12)$ & $0.6100(11)$ & $0.082(3)$ & 0.34 \\
\hline $\mathrm{H} 82 \mathrm{~A}$ & -0.1962 & 0.0714 & 0.6444 & $0.098 *$ & 0.34 \\
\hline $\mathrm{C} 83 \mathrm{~A}$ & $-0.1209(15)$ & $0.1416(12)$ & $0.6139(7)$ & $0.082(3)$ & 0.34 \\
\hline H83A & -0.0979 & 0.1629 & 0.6505 & $0.099 *$ & 0.34 \\
\hline C84A & $-0.0965(12)$ & $0.1648(9)$ & $0.5594(6)$ & $0.081(2)$ & 0.34 \\
\hline H84A & -0.0572 & 0.2019 & 0.5609 & $0.097 *$ & 0.34 \\
\hline N1 & $0.27646(17)$ & $0.24607(14)$ & $0.76671(13)$ & $0.0330(6)$ & \\
\hline $\mathrm{N} 2$ & $0.37143(17)$ & $0.14219(14)$ & $0.79170(13)$ & $0.0302(6)$ & \\
\hline N3 & $0.28561(17)$ & $0.22388(14)$ & 0.93075 (12) & $0.0312(6)$ & \\
\hline N4 & $0.42751(17)$ & $0.12499(14)$ & $0.93553(12)$ & $0.0307(6)$ & \\
\hline N5 & $0.13044(18)$ & $0.15645(14)$ & $0.76514(14)$ & $0.0337(6)$ & \\
\hline N6 & $0.18439(17)$ & $0.00730(14)$ & $0.76635(13)$ & $0.0308(6)$ & \\
\hline N7 & $0.13358(18)$ & $0.14323(15)$ & $0.93093(13)$ & $0.0345(6)$ & \\
\hline N8 & $0.20644(18)$ & $0.03278(15)$ & $0.90921(13)$ & $0.0330(6)$ & \\
\hline N9 & $0.45806(17)$ & $-0.03173(14)$ & $0.89580(12)$ & $0.0301(6)$ & \\
\hline N10 & $0.34564(17)$ & $-0.08566(14)$ & $0.81453(13)$ & $0.0329(6)$ & \\
\hline $\mathrm{O} 1$ & $0.21502(15)$ & $0.29635(12)$ & $0.75199(13)$ & $0.0424(6)$ & \\
\hline $\mathrm{O} 2$ & $0.41168(14)$ & $0.07989(11)$ & $0.80519(11)$ & $0.0333(5)$ & \\
\hline $\mathrm{O} 3$ & $0.22535(15)$ & $0.27495(13)$ & $0.92507(12)$ & $0.0399(6)$ & \\
\hline $\mathrm{O} 4$ & $0.34924(14)$ & $0.09770(12)$ & $0.91047(11)$ & $0.0342(5)$ & \\
\hline $\mathrm{O} 5$ & $0.09813(16)$ & $0.22254(13)$ & $0.76779(14)$ & $0.0454(7)$ & \\
\hline O6 & $0.24766(14)$ & $0.05433(12)$ & $0.79020(11)$ & $0.0331(5)$ & \\
\hline $\mathrm{O} 7$ & $0.10615(16)$ & $0.20776(14)$ & $0.94544(13)$ & $0.0445(6)$ & \\
\hline O8 & $0.25669(15)$ & $-0.02059(12)$ & $0.89833(11)$ & $0.0361(5)$ & \\
\hline O9 & $0.52088(17)$ & $0.00669(14)$ & $0.93202(12)$ & $0.0404(6)$ & \\
\hline $\mathrm{O} 10$ & $0.27559(16)$ & $-0.11284(13)$ & $0.77446(12)$ & $0.0395(6)$ & \\
\hline Ni1 & $0.25135(3)$ & $0.14989(2)$ & $0.77620(2)$ & 0.03005 (11) & \\
\hline $\mathrm{Ni} 2$ & $0.24612(3)$ & $0.12803(2)$ & $0.92204(2)$ & $0.03014(11)$ & \\
\hline $\mathrm{Ni3}$ & $0.34675(3)$ & $0.01380(2)$ & $0.85238(2)$ & $0.02978(11)$ & \\
\hline H1A & 0.149 (4) & $0.262(3)$ & $0.761(3)$ & $0.10(2)^{*}$ & \\
\hline $\mathrm{H} 2 \mathrm{~A}$ & $0.166(3)$ & $0.244(3)$ & $0.935(2)$ & $0.081(16)^{*}$ & \\
\hline $\mathrm{H} 3 \mathrm{~A}$ & $0.488(4)$ & $0.043(3)$ & $0.933(3)$ & $0.09(2)^{*}$ & \\
\hline $\mathrm{H} 4 \mathrm{~A}$ & $0.233(3)$ & -0.070 & $0.770(2)$ & $0.095(18)^{*}$ & \\
\hline
\end{tabular}


Atomic displacement parameters $\left(\AA^{2}\right)$

\begin{tabular}{|c|c|c|c|c|c|c|}
\hline & $U^{11}$ & $U^{22}$ & $U^{33}$ & $U^{12}$ & $U^{13}$ & $U^{23}$ \\
\hline $\mathrm{C} 1$ & $0.0263(16)$ & $0.0267(15)$ & $0.0376(17)$ & $-0.0007(13)$ & $0.0076(13)$ & $0.0027(13)$ \\
\hline $\mathrm{C} 2$ & $0.0271(16)$ & $0.0272(15)$ & $0.0329(16)$ & $0.0010(13)$ & 0.0088 (13) & $0.0019(13)$ \\
\hline $\mathrm{C} 3$ & $0.0274(16)$ & $0.0301(16)$ & $0.0318(16)$ & $-0.0026(13)$ & $0.0120(13)$ & $-0.0014(13)$ \\
\hline $\mathrm{C} 4$ & $0.0296(17)$ & $0.0359(18)$ & $0.0379(18)$ & $0.0044(14)$ & 0.0095 (14) & $0.0015(14)$ \\
\hline $\mathrm{C} 5$ & $0.0292(18)$ & $0.047(2)$ & 0.0414 (19) & $0.0061(16)$ & $0.0076(15)$ & 0.0005 (16) \\
\hline C6 & 0.0243 (17) & $0.055(2)$ & $0.046(2)$ & $-0.0013(16)$ & $0.0035(15)$ & $-0.0013(18)$ \\
\hline $\mathrm{C} 7$ & $0.0321(19)$ & $0.045(2)$ & $0.046(2)$ & $-0.0098(16)$ & $0.0084(15)$ & $-0.0027(17)$ \\
\hline $\mathrm{C} 8$ & $0.0286(17)$ & $0.0349(17)$ & $0.0343(17)$ & $-0.0010(14)$ & $0.0122(13)$ & $-0.0019(14)$ \\
\hline C9 & $0.0319(17)$ & $0.0291(16)$ & $0.0334(16)$ & $-0.0043(13)$ & $0.0125(13)$ & $-0.0049(13)$ \\
\hline $\mathrm{C} 10$ & $0.0361(19)$ & $0.0343(18)$ & 0.0448 (19) & $-0.0093(15)$ & $0.0173(15)$ & $-0.0043(15)$ \\
\hline C11 & 0.049 (2) & $0.0278(17)$ & $0.048(2)$ & $-0.0082(16)$ & $0.0199(17)$ & $0.0011(15)$ \\
\hline C12 & $0.048(2)$ & $0.0265(16)$ & $0.046(2)$ & $0.0013(15)$ & $0.0182(17)$ & $0.0047(15)$ \\
\hline C13 & $0.0344(18)$ & $0.0274(16)$ & $0.0396(18)$ & $0.0021(14)$ & $0.0104(14)$ & $0.0029(14)$ \\
\hline C14 & $0.0313(17)$ & $0.0237(15)$ & $0.0354(16)$ & $-0.0018(13)$ & $0.0118(13)$ & $0.0005(13)$ \\
\hline C15 & $0.0300(17)$ & $0.0285(16)$ & $0.0298(15)$ & $-0.0013(13)$ & $0.0080(13)$ & $-0.0011(13)$ \\
\hline $\mathrm{C} 16$ & $0.0288(16)$ & $0.0261(15)$ & $0.0309(15)$ & $-0.0018(13)$ & $0.0081(13)$ & $-0.0004(12)$ \\
\hline $\mathrm{C} 17$ & $0.0282(16)$ & $0.0318(16)$ & $0.0285(15)$ & $-0.0034(13)$ & $0.0072(12)$ & $-0.0013(13)$ \\
\hline $\mathrm{C} 18$ & 0.0335 (18) & $0.0324(17)$ & $0.0321(16)$ & $-0.0039(14)$ & $0.0062(14)$ & $-0.0003(14)$ \\
\hline C19 & 0.0315 (18) & $0.043(2)$ & $0.0338(17)$ & $0.0031(15)$ & $0.0052(14)$ & $0.0020(15)$ \\
\hline $\mathrm{C} 20$ & $0.0312(18)$ & $0.049(2)$ & 0.0400 (19) & $-0.0079(16)$ & $-0.0001(15)$ & $-0.0026(16)$ \\
\hline $\mathrm{C} 21$ & $0.039(2)$ & 0.0372 (19) & 0.0425 (19) & $-0.0116(16)$ & 0.0077 (16) & $-0.0027(16)$ \\
\hline $\mathrm{C} 22$ & $0.0322(17)$ & 0.0329 (17) & $0.0312(16)$ & $-0.0055(14)$ & 0.0095 (13) & $-0.0022(13)$ \\
\hline $\mathrm{C} 23$ & 0.0345 (18) & 0.0299 (16) & $0.0328(16)$ & $-0.0010(14)$ & $0.0116(14)$ & $-0.0016(13)$ \\
\hline $\mathrm{C} 24$ & $0.040(2)$ & $0.0316(17)$ & $0.047(2)$ & $-0.0064(15)$ & $0.0132(16)$ & $-0.0014(15)$ \\
\hline $\mathrm{C} 25$ & $0.053(2)$ & 0.0287 (17) & $0.050(2)$ & $-0.0040(16)$ & $0.0183(18)$ & $-0.0005(16)$ \\
\hline $\mathrm{C} 26$ & $0.048(2)$ & $0.0298(17)$ & $0.046(2)$ & $0.0042(16)$ & $0.0131(17)$ & $0.0056(15)$ \\
\hline $\mathrm{C} 27$ & 0.039 (2) & $0.0315(17)$ & $0.0391(18)$ & $0.0016(15)$ & 0.0109 (15) & $0.0002(14)$ \\
\hline $\mathrm{C} 28$ & 0.0335 (17) & 0.0283 (16) & $0.0341(17)$ & $-0.0011(14)$ & $0.0123(14)$ & $-0.0011(13)$ \\
\hline $\mathrm{C} 29$ & $0.0243(16)$ & $0.0294(16)$ & $0.0328(16)$ & $0.0010(13)$ & $0.0043(12)$ & $0.0051(13)$ \\
\hline $\mathrm{C} 30$ & 0.0267 (16) & $0.0299(16)$ & $0.0355(17)$ & $-0.0019(13)$ & 0.0053 (13) & $0.0030(13)$ \\
\hline C31 & $0.0344(18)$ & $0.0314(17)$ & $0.0359(17)$ & $-0.0036(14)$ & $0.0026(14)$ & $0.0023(14)$ \\
\hline $\mathrm{C} 32$ & $0.037(2)$ & $0.041(2)$ & $0.048(2)$ & $-0.0034(16)$ & $0.0033(16)$ & $-0.0064(17)$ \\
\hline C33 & $0.050(3)$ & $0.040(2)$ & $0.064(3)$ & $-0.0043(19)$ & $0.001(2)$ & -0.0108 (19) \\
\hline $\mathrm{C} 34$ & $0.047(2)$ & $0.042(2)$ & $0.065(3)$ & $-0.0139(19)$ & $-0.006(2)$ & $-0.003(2)$ \\
\hline C35 & $0.034(2)$ & $0.043(2)$ & $0.060(2)$ & $-0.0075(17)$ & $-0.0004(17)$ & 0.0005 (19) \\
\hline $\mathrm{C} 36$ & $0.0312(18)$ & 0.0355 (18) & 0.0403 (19) & $-0.0055(14)$ & 0.0007 (14) & $0.0057(15)$ \\
\hline $\mathrm{C} 37$ & 0.0289 (17) & 0.0405 (19) & $0.0374(18)$ & $-0.0029(15)$ & $0.0040(14)$ & $0.0083(15)$ \\
\hline $\mathrm{C} 38$ & 0.0283 (18) & $0.052(2)$ & 0.049 (2) & $-0.0044(16)$ & $0.0051(16)$ & $0.0110(18)$ \\
\hline C39 & 0.0297 (19) & $0.063(3)$ & $0.048(2)$ & $0.0074(18)$ & $0.0115(16)$ & $0.0120(19)$ \\
\hline $\mathrm{C} 40$ & $0.033(2)$ & $0.057(2)$ & $0.047(2)$ & $0.0098(18)$ & $0.0127(16)$ & $0.0050(18)$ \\
\hline $\mathrm{C} 41$ & 0.0339 (19) & 0.0388 (19) & $0.046(2)$ & $0.0010(15)$ & $0.0088(15)$ & $-0.0008(16)$ \\
\hline $\mathrm{C} 42$ & $0.0255(16)$ & $0.0349(17)$ & $0.0357(17)$ & $0.0006(13)$ & $0.0043(13)$ & $0.0063(14)$ \\
\hline $\mathrm{C} 43$ & $0.0300(17)$ & $0.0390(18)$ & $0.0344(17)$ & $-0.0011(14)$ & $0.0059(13)$ & $0.0044(14)$ \\
\hline $\mathrm{C} 44$ & $0.0328(18)$ & $0.0368(18)$ & $0.0328(17)$ & $-0.0041(14)$ & $0.0035(14)$ & $0.0078(14)$ \\
\hline $\mathrm{C} 45$ & $0.038(2)$ & $0.0362(18)$ & $0.0372(18)$ & $-0.0071(15)$ & $0.0031(15)$ & $0.0051(15)$ \\
\hline
\end{tabular}




\begin{tabular}{|c|c|c|c|c|c|c|}
\hline $\mathrm{C} 46$ & $0.043(2)$ & $0.041(2)$ & $0.044(2)$ & -0.0107 (17) & $0.0031(16)$ & $0.0024(16)$ \\
\hline $\mathrm{C} 47$ & $0.063(3)$ & $0.044(2)$ & $0.058(3)$ & $-0.017(2)$ & $0.008(2)$ & $-0.005(2)$ \\
\hline $\mathrm{C} 48$ & $0.056(3)$ & $0.058(3)$ & $0.071(3)$ & $-0.030(2)$ & $0.001(2)$ & $-0.004(2)$ \\
\hline C49 & $0.043(2)$ & $0.054(3)$ & $0.060(3)$ & $-0.017(2)$ & 0.0017 (19) & $0.002(2)$ \\
\hline $\mathrm{C} 50$ & $0.038(2)$ & $0.047(2)$ & $0.0387(19)$ & $-0.0151(17)$ & $0.0003(15)$ & $0.0102(17)$ \\
\hline $\mathrm{C} 51$ & $0.0311(19)$ & $0.053(2)$ & $0.0361(18)$ & $-0.0055(16)$ & $0.0019(14)$ & $0.0130(16)$ \\
\hline $\mathrm{C} 52$ & $0.037(2)$ & $0.064(3)$ & $0.049(2)$ & -0.0115 (19) & $0.0057(17)$ & $0.016(2)$ \\
\hline $\mathrm{C} 53$ & $0.030(2)$ & $0.078(3)$ & $0.051(2)$ & $0.002(2)$ & $0.0115(17)$ & $0.019(2)$ \\
\hline C54 & $0.035(2)$ & 0.074 & $0.046(2)$ & $0.005(2)$ & $0.0135(17)$ & $0.011(2)$ \\
\hline $\mathrm{C} 55$ & $0.0318(19)$ & $0.054(2)$ & $0.045(2)$ & 0.0010 & $0.0108(16)$ & $0.0073(18)$ \\
\hline $\mathrm{C} 56$ & $0.0281(17)$ & $0.050(2)$ & $0.0341(17)$ & $-0.0037(16)$ & $0.0025(14)$ & $0.0097(16)$ \\
\hline C57 & $0.0320(17)$ & $0.0263(15)$ & $0.0364(17)$ & $0.0007(13)$ & $0.0102(14)$ & $0.0033(13)$ \\
\hline $\mathrm{C} 58$ & $0.0323(17)$ & $0.0262(15)$ & $0.0341(16)$ & $0.0016(13)$ & $0.0095(13)$ & $0.0031(13)$ \\
\hline C59 & $0.0381(18)$ & $0.0273(16)$ & $0.0303(16)$ & $0.0003(14)$ & $0.0061(14)$ & $0.0001(13)$ \\
\hline C60 & $0.042(2)$ & $0.0313(18)$ & $0.048(2)$ & $-0.0002(15)$ & $0.0036(16)$ & $0.0007(16)$ \\
\hline C61 & $0.065(3)$ & $0.0309(19)$ & $0.050(2)$ & $-0.0080(19)$ & $-0.003(2)$ & $-0.0026(17)$ \\
\hline C62 & $0.076(3)$ & $0.0271(18)$ & $0.053(2)$ & $0.0001(19)$ & $0.003(2)$ & $-0.0054(17)$ \\
\hline $\mathrm{C} 63$ & $0.058(3)$ & $0.0330(19)$ & $0.053(2)$ & $0.0136(18)$ & $0.0061(19)$ & $0.0004(17)$ \\
\hline C64 & $0.047(2)$ & $0.0299(17)$ & $0.0355(18)$ & $0.0032(15)$ & $0.0124(15)$ & $0.0008(14)$ \\
\hline C65 & $0.0396(19)$ & $0.0345(18)$ & $0.0382(18)$ & $0.0070(15)$ & $0.0130(15)$ & $0.0062(15)$ \\
\hline C66 & $0.045(2)$ & $0.039(2)$ & $0.054(2)$ & $0.0106(17)$ & $0.0135(18)$ & $0.0073(17)$ \\
\hline C67 & $0.038(2)$ & $0.047(2)$ & $0.060(2)$ & $0.0133(18)$ & $0.0112(18)$ & $0.0134(19)$ \\
\hline C68 & $0.0332(19)$ & $0.047(2)$ & $0.050(2)$ & $0.0003(16)$ & $0.0055(16)$ & $0.0147(18)$ \\
\hline C69 & $0.0358(19)$ & $0.0345(18)$ & $0.0411(19)$ & $0.0052(15)$ & $0.0074(15)$ & $0.0093(15)$ \\
\hline $\mathrm{C} 70$ & $0.0312(17)$ & $0.0303(16)$ & $0.0356(17)$ & $0.0021(14)$ & $0.0085(14)$ & $0.0062(14)$ \\
\hline C71 & $0.055(3)$ & $0.108(5)$ & $0.138(6)$ & 0.015 & $0.025(4)$ & $0.052(4)$ \\
\hline $\mathrm{C} 72$ & $0.047(3)$ & $0.054(3)$ & 0.080 & $0.000(2)$ & $0.019(2)$ & $0.015(2)$ \\
\hline $\mathrm{C} 73$ & $0.055(3)$ & $0.052(2)$ & $0.050(2)$ & $-0.001(2)$ & 0.0157 (19) & $0.0116(19)$ \\
\hline C74 & 0.059 (3) & $0.045(2)$ & $0.053(2)$ & $0.000(2)$ & $0.026(2)$ & $-0.0001(19)$ \\
\hline $\mathrm{C} 75$ & $0.054(3)$ & $0.045(2)$ & $0.053(2)$ & $0.0034(19)$ & $0.014(2)$ & $0.0046(19)$ \\
\hline C76 & 0.070 & $0.074(3)$ & $0.052(3)$ & 0.018 & $0.025(2)$ & $0.016(2)$ \\
\hline C77 & 0.075 & $0.083(4)$ & $0.071(3)$ & 0.017 (3) & $0.045(3)$ & 0.020 \\
\hline $\mathrm{C} 78$ & $0.110(6)$ & $0.077(5)$ & $0.074(5)$ & $-0.002(5)$ & $0.018(5)$ & -0.001 \\
\hline C79 & $0.081(4)$ & $0.068(4)$ & 0.068 & $0.006(4)$ & 0.014 & -0.012 \\
\hline $\mathrm{C} 80$ & $0.100(5)$ & 0.069 (4) & 0.067 (4) & 0.005 (4) & $0.006(4)$ & -0.005 \\
\hline C81 & $0.105(5)$ & $0.069(4)$ & 0.075 (4) & 0.007 (4) & -0.001 & $0.004(4)$ \\
\hline $\mathrm{C} 82$ & $0.097(5)$ & $0.070(5)$ & 0.069 (4) & 0.009 (4) & -0.005 & -0.009 \\
\hline $\mathrm{C} 83$ & 0.088 (4) & $0.078(5)$ & 0.080 & $0.007(4)$ & 0.007 (4) & -0.015 \\
\hline $\mathrm{C} 84$ & $0.080(4)$ & 0.075 & $0.076(4)$ & 0.010 & $0.018(4)$ & -0.010 \\
\hline C78A & $0.087(8)$ & $0.089(8)$ & $0.067(7)$ & $0.002(7)$ & $0.035(6)$ & $0.000(7)$ \\
\hline C79A & $0.090(5)$ & $0.074(5)$ & $0.074(4)$ & 0.010 & 0.015 (4) & -0.008 \\
\hline C81A & $0.098(6)$ & $0.074(6)$ & $0.070(5)$ & $0.002(5)$ & $0.010(5)$ & $-0.003(5)$ \\
\hline $\mathrm{C} 80 \mathrm{~A}$ & $0.093(6)$ & $0.070(5)$ & $0.072(5)$ & $0.001(5)$ & $0.014(5)$ & -0.005 \\
\hline C82A & $0.097(6)$ & $0.072(5)$ & $0.071(5)$ & $0.007(5)$ & $0.004(5)$ & $0.000(4)$ \\
\hline $\mathrm{C} 83 \mathrm{~A}$ & $0.095(6)$ & $0.074(5)$ & $0.071(4)$ & $0.006(5)$ & $-0.003(5)$ & -0.005 \\
\hline C84A & $0.090(5)$ & $0.074(5)$ & 0.075 & $0.008(4)$ & 0.007 (4) & -0.009 \\
\hline N1 & $0.0260(14)$ & $0.0272(14)$ & $0.0453(16)$ & $0.0056(11)$ & $0.0061(12)$ & $0.0058(12)$ \\
\hline $\mathrm{N} 2$ & $0.0292(14)$ & $0.0235(13)$ & $0.0386(15)$ & $0.0031(11)$ & $0.0087(12)$ & $0.0020(11)$ \\
\hline
\end{tabular}




\begin{tabular}{lllllll} 
N3 & $0.0283(14)$ & $0.0289(14)$ & $0.0366(15)$ & $0.0044(11)$ & $0.0072(11)$ & $0.0010(11)$ \\
N4 & $0.0267(14)$ & $0.0288(13)$ & $0.0366(14)$ & $-0.0035(11)$ & $0.0064(11)$ & $-0.0041(11)$ \\
N5 & $0.0284(15)$ & $0.0259(13)$ & $0.0478(17)$ & $0.0024(11)$ & $0.0104(12)$ & $0.0030(12)$ \\
N6 & $0.0275(14)$ & $0.0251(13)$ & $0.0394(15)$ & $-0.0040(11)$ & $0.0060(12)$ & $0.0006(11)$ \\
N7 & $0.0304(15)$ & $0.0345(15)$ & $0.0379(15)$ & $-0.0016(12)$ & $0.0058(12)$ & $0.0002(12)$ \\
N8 & $0.0307(15)$ & $0.0301(14)$ & $0.0389(15)$ & $-0.0007(12)$ & $0.0084(12)$ & $0.0027(12)$ \\
N9 & $0.0279(14)$ & $0.0247(13)$ & $0.0365(14)$ & $-0.0019(11)$ & $0.0034(11)$ & $0.0023(11)$ \\
N10 & $0.0294(15)$ & $0.0264(13)$ & $0.0415(16)$ & $-0.0013(11)$ & $0.0039(12)$ & $-0.0017(12)$ \\
O1 & $0.0271(13)$ & $0.0255(12)$ & $0.0740(19)$ & $0.0064(10)$ & $0.0091(12)$ & $0.0141(12)$ \\
O2 & $0.0295(12)$ & $0.0231(11)$ & $0.0480(14)$ & $0.0047(9)$ & $0.0098(10)$ & $0.0017(10)$ \\
O3 & $0.0292(13)$ & $0.0282(12)$ & $0.0627(17)$ & $0.0058(10)$ & $0.0104(11)$ & $0.0020(11)$ \\
O4 & $0.0255(12)$ & $0.0296(12)$ & $0.0474(14)$ & $-0.0045(9)$ & $0.0072(10)$ & $-0.0069(10)$ \\
O5 & $0.0288(13)$ & $0.0255(12)$ & $0.084(2)$ & $0.0048(10)$ & $0.0163(13)$ & $0.0077(13)$ \\
O6 & $0.0249(12)$ & $0.0255(11)$ & $0.0468(14)$ & $-0.0039(9)$ & $0.0021(10)$ & $0.0009(10)$ \\
O7 & $0.0333(14)$ & $0.0368(14)$ & $0.0663(18)$ & $0.0036(11)$ & $0.0169(12)$ & $-0.0043(13)$ \\
O8 & $0.0344(13)$ & $0.0292(12)$ & $0.0449(14)$ & $-0.0010(10)$ & $0.0087(11)$ & $0.0005(10)$ \\
O9 & $0.0337(14)$ & $0.0317(13)$ & $0.0506(15)$ & $-0.0002(11)$ & $-0.0038(11)$ & $-0.0058(11)$ \\
O10 & $0.0349(14)$ & $0.0309(12)$ & $0.0471(15)$ & $0.0019(11)$ & $-0.0049(11)$ & $-0.0071(11)$ \\
Ni1 & $0.0237(2)$ & $0.0232(2)$ & $0.0434(2)$ & $0.00088(16)$ & $0.00702(17)$ & $0.00455(17)$ \\
Ni2 & $0.0251(2)$ & $0.0275(2)$ & $0.0382(2)$ & $-0.00170(16)$ & $0.00718(17)$ & $-0.00033(17)$ \\
Ni3 & $0.0274(2)$ & $0.0223(2)$ & $0.0386(2)$ & $0.00040(16)$ & $0.00435(17)$ & $0.00011(17)$ \\
& & & & & & \\
\hline
\end{tabular}

Geometric parameters $\left(\AA,{ }^{\circ}\right)$

\begin{tabular}{llll}
\hline $\mathrm{C} 1-\mathrm{N} 1$ & $1.310(4)$ & $\mathrm{C} 55-\mathrm{H} 55$ & 0.9300 \\
$\mathrm{C} 1-\mathrm{C} 14$ & $1.461(4)$ & $\mathrm{C} 57-\mathrm{N} 9$ & $1.310(4)$ \\
$\mathrm{C} 1-\mathrm{C} 2$ & $1.464(4)$ & $\mathrm{C} 57-\mathrm{C} 70$ & $1.465(5)$ \\
$\mathrm{C} 2-\mathrm{N} 2$ & $1.322(4)$ & $\mathrm{C} 57-\mathrm{C} 58$ & $1.495(5)$ \\
$\mathrm{C} 2-\mathrm{C} 3$ & $1.462(4)$ & $\mathrm{C} 58-\mathrm{N} 10$ & $1.299(4)$ \\
$\mathrm{C} 3-\mathrm{C} 4$ & $1.410(5)$ & $\mathrm{C} 58-\mathrm{C} 59$ & $1.467(4)$ \\
$\mathrm{C} 3-\mathrm{C} 8$ & $1.418(4)$ & $\mathrm{C} 59-\mathrm{C} 60$ & $1.406(5)$ \\
$\mathrm{C} 4-\mathrm{C} 5$ & $1.373(5)$ & $\mathrm{C} 59-\mathrm{C} 64$ & $1.408(5)$ \\
$\mathrm{C} 4-\mathrm{H} 4$ & 0.9300 & $\mathrm{C} 60-\mathrm{C} 61$ & $1.372(5)$ \\
$\mathrm{C} 5-\mathrm{C} 6$ & $1.383(5)$ & $\mathrm{C} 60-\mathrm{H} 60$ & 0.9300 \\
$\mathrm{C} 5-\mathrm{H} 5$ & 0.9300 & $\mathrm{C} 61-\mathrm{C} 62$ & $1.368(6)$ \\
$\mathrm{C} 6-\mathrm{C} 7$ & $1.374(5)$ & $\mathrm{C} 61-\mathrm{H} 61$ & 0.9300 \\
$\mathrm{C} 6-\mathrm{H} 6$ & 0.9300 & $\mathrm{C} 62-\mathrm{C} 63$ & $1.380(6)$ \\
$\mathrm{C} 7-\mathrm{C} 8$ & $1.400(5)$ & $\mathrm{C} 62-\mathrm{H} 62$ & 0.9300 \\
$\mathrm{C} 7-\mathrm{H} 7$ & 0.9300 & $\mathrm{C} 63-\mathrm{C} 64$ & $1.399(5)$ \\
$\mathrm{C} 8-\mathrm{C} 9$ & $1.479(5)$ & $\mathrm{C} 63-\mathrm{H} 63$ & 0.9300 \\
$\mathrm{C} 9-\mathrm{C} 10$ & $1.406(4)$ & $\mathrm{C} 64-\mathrm{C} 65$ & $1.481(5)$ \\
$\mathrm{C} 9-\mathrm{C} 14$ & $1.412(5)$ & $\mathrm{C} 65-\mathrm{C} 66$ & $1.400(5)$ \\
$\mathrm{C} 10-\mathrm{C} 11$ & $1.373(5)$ & $\mathrm{C} 65-\mathrm{C} 70$ & $1.416(5)$ \\
$\mathrm{C} 10-\mathrm{H} 10$ & 0.9300 & $\mathrm{C} 66-\mathrm{C} 67$ & $1.364(6)$ \\
$\mathrm{C} 11-\mathrm{C} 12$ & $1.387(5)$ & $\mathrm{C} 66-\mathrm{H} 66$ & 0.9300 \\
$\mathrm{C} 11-\mathrm{H} 11$ & 0.9300 & $\mathrm{C} 67-\mathrm{C} 68$ & $1.379(6)$ \\
$\mathrm{C} 12-\mathrm{C} 13$ & $1.384(5)$ & $\mathrm{C} 67-\mathrm{H} 67$ & 0.9300 \\
$\mathrm{C} 12-\mathrm{H} 12$ & 0.9300 & $\mathrm{C} 68-\mathrm{C} 69$ & $1.378(5)$ \\
& & &
\end{tabular}




\begin{tabular}{|c|c|c|c|}
\hline $\mathrm{C} 13-\mathrm{C} 14$ & $1.408(4)$ & C68-H68 & 0.9300 \\
\hline C13-H13 & 0.9300 & $\mathrm{C} 69-\mathrm{C} 70$ & $1.420(5)$ \\
\hline $\mathrm{C} 15-\mathrm{N} 3$ & $1.321(4)$ & C69-H69 & 0.9300 \\
\hline $\mathrm{C} 15-\mathrm{C} 16$ & $1.468(4)$ & $\mathrm{C} 71-\mathrm{C} 72$ & $1.517(7)$ \\
\hline $\mathrm{C} 15-\mathrm{C} 28$ & $1.479(4)$ & $\mathrm{C} 71-\mathrm{H} 71 \mathrm{~A}$ & 0.9600 \\
\hline $\mathrm{C} 16-\mathrm{N} 4$ & $1.290(4)$ & $\mathrm{C} 71-\mathrm{H} 71 \mathrm{~B}$ & 0.9600 \\
\hline $\mathrm{C} 16-\mathrm{C} 17$ & $1.481(4)$ & $\mathrm{C} 71-\mathrm{H} 71 \mathrm{C}$ & 0.9600 \\
\hline $\mathrm{C} 17-\mathrm{C} 22$ & $1.396(4)$ & $\mathrm{C} 72-\mathrm{C} 73$ & $1.387(6)$ \\
\hline $\mathrm{C} 17-\mathrm{C} 18$ & $1.401(5)$ & $\mathrm{C} 72-\mathrm{C} 77$ & $1.388(7)$ \\
\hline $\mathrm{C} 18-\mathrm{C} 19$ & $1.380(5)$ & $\mathrm{C} 73-\mathrm{C} 74$ & $1.369(6)$ \\
\hline $\mathrm{C} 18-\mathrm{H} 18$ & 0.9300 & $\mathrm{C} 73-\mathrm{H} 73$ & 0.9300 \\
\hline $\mathrm{C} 19-\mathrm{C} 20$ & $1.390(5)$ & $\mathrm{C} 74-\mathrm{C} 75$ & $1.382(6)$ \\
\hline C19-H19 & 0.9300 & C74-H74 & 0.9300 \\
\hline $\mathrm{C} 20-\mathrm{C} 21$ & $1.372(5)$ & $\mathrm{C} 75-\mathrm{C} 76$ & $1.365(6)$ \\
\hline $\mathrm{C} 20-\mathrm{H} 20$ & 0.9300 & C75-H75 & 0.9300 \\
\hline $\mathrm{C} 21-\mathrm{C} 22$ & $1.410(5)$ & $\mathrm{C} 76-\mathrm{C} 77$ & $1.377(7)$ \\
\hline $\mathrm{C} 21-\mathrm{H} 21$ & 0.9300 & C76-H76 & 0.9300 \\
\hline $\mathrm{C} 22-\mathrm{C} 23$ & $1.469(5)$ & C77-H77 & 0.9300 \\
\hline $\mathrm{C} 23-\mathrm{C} 24$ & $1.399(5)$ & $\mathrm{C} 78-\mathrm{C} 79$ & $1.470(10)$ \\
\hline $\mathrm{C} 23-\mathrm{C} 28$ & $1.424(5)$ & $\mathrm{C} 78-\mathrm{H} 78 \mathrm{~A}$ & 0.9600 \\
\hline $\mathrm{C} 24-\mathrm{C} 25$ & $1.376(5)$ & $\mathrm{C} 78-\mathrm{H} 78 \mathrm{~B}$ & 0.9600 \\
\hline $\mathrm{C} 24-\mathrm{H} 24$ & 0.9300 & $\mathrm{C} 78-\mathrm{H} 78 \mathrm{C}$ & 0.9600 \\
\hline $\mathrm{C} 25-\mathrm{C} 26$ & $1.378(5)$ & $\mathrm{C} 79-\mathrm{C} 80$ & $1.386(6)$ \\
\hline $\mathrm{C} 25-\mathrm{H} 25$ & 0.9300 & $\mathrm{C} 79-\mathrm{C} 84$ & $1.401(6)$ \\
\hline $\mathrm{C} 26-\mathrm{C} 27$ & $1.384(5)$ & $\mathrm{C} 80-\mathrm{C} 81$ & $1.386(7)$ \\
\hline $\mathrm{C} 26-\mathrm{H} 26$ & 0.9300 & $\mathrm{C} 80-\mathrm{H} 80$ & 0.9300 \\
\hline $\mathrm{C} 27-\mathrm{C} 28$ & $1.402(5)$ & $\mathrm{C} 81-\mathrm{C} 82$ & $1.390(7)$ \\
\hline $\mathrm{C} 27-\mathrm{H} 27$ & 0.9300 & $\mathrm{C} 81-\mathrm{H} 81$ & 0.9300 \\
\hline $\mathrm{C} 29-\mathrm{N} 5$ & $1.319(4)$ & $\mathrm{C} 82-\mathrm{C} 83$ & $1.386(7)$ \\
\hline $\mathrm{C} 29-\mathrm{C} 30$ & $1.465(4)$ & $\mathrm{C} 82-\mathrm{H} 82$ & 0.9300 \\
\hline $\mathrm{C} 29-\mathrm{C} 42$ & $1.479(4)$ & $\mathrm{C} 83-\mathrm{C} 84$ & $1.384(6)$ \\
\hline $\mathrm{C} 30-\mathrm{N} 6$ & $1.295(4)$ & $\mathrm{C} 83-\mathrm{H} 83$ & 0.9300 \\
\hline $\mathrm{C} 30-\mathrm{C} 31$ & $1.472(5)$ & $\mathrm{C} 84-\mathrm{H} 84$ & 0.9300 \\
\hline $\mathrm{C} 31-\mathrm{C} 32$ & $1.394(5)$ & $\mathrm{C} 78 \mathrm{~A}-\mathrm{C} 79 \mathrm{~A}$ & $1.523(18)$ \\
\hline $\mathrm{C} 31-\mathrm{C} 36$ & $1.399(5)$ & $\mathrm{C} 78 \mathrm{~A}-\mathrm{H} 78 \mathrm{D}$ & 0.9600 \\
\hline $\mathrm{C} 32-\mathrm{C} 33$ & $1.384(5)$ & $\mathrm{C} 78 \mathrm{~A}-\mathrm{H} 78 \mathrm{E}$ & 0.9600 \\
\hline $\mathrm{C} 32-\mathrm{H} 32$ & 0.9300 & $\mathrm{C} 78 \mathrm{~A}-\mathrm{H} 78 \mathrm{~F}$ & 0.9600 \\
\hline $\mathrm{C} 33-\mathrm{C} 34$ & $1.367(6)$ & $\mathrm{C} 79 \mathrm{~A}-\mathrm{C} 84 \mathrm{~A}$ & $1.32(2)$ \\
\hline $\mathrm{C} 33-\mathrm{H} 33$ & 0.9300 & $\mathrm{C} 79 \mathrm{~A}-\mathrm{C} 80 \mathrm{~A}$ & $1.382(8)$ \\
\hline $\mathrm{C} 34-\mathrm{C} 35$ & $1.372(6)$ & $\mathrm{C} 81 \mathrm{~A}-\mathrm{C} 82 \mathrm{~A}$ & $1.26(3)$ \\
\hline $\mathrm{C} 34-\mathrm{H} 34$ & 0.9300 & $\mathrm{C} 81 \mathrm{~A}-\mathrm{C} 80 \mathrm{~A}$ & $1.46(2)$ \\
\hline $\mathrm{C} 35-\mathrm{C} 36$ & $1.410(5)$ & $\mathrm{C} 81 \mathrm{~A}-\mathrm{H} 81 \mathrm{~A}$ & 0.9300 \\
\hline $\mathrm{C} 35-\mathrm{H} 35$ & 0.9300 & $\mathrm{C} 80 \mathrm{~A}-\mathrm{H} 80 \mathrm{~A}$ & 0.9300 \\
\hline $\mathrm{C} 36-\mathrm{C} 37$ & $1.469(5)$ & $\mathrm{C} 82 \mathrm{~A}-\mathrm{C} 83 \mathrm{~A}$ & $1.36(3)$ \\
\hline $\mathrm{C} 37-\mathrm{C} 38$ & $1.403(5)$ & $\mathrm{C} 82 \mathrm{~A}-\mathrm{H} 82 \mathrm{~A}$ & 0.9300 \\
\hline C $37-\mathrm{C} 42$ & $1.420(5)$ & $\mathrm{C} 83 \mathrm{~A}-\mathrm{C} 84 \mathrm{~A}$ & $1.44(2)$ \\
\hline $\mathrm{C} 38-\mathrm{C} 39$ & $1.375(6)$ & $\mathrm{C} 83 \mathrm{~A}-\mathrm{H} 83 \mathrm{~A}$ & 0.9300 \\
\hline $\mathrm{C} 38-\mathrm{H} 38$ & 0.9300 & $\mathrm{C} 84 \mathrm{~A}-\mathrm{H} 84 \mathrm{~A}$ & 0.9300 \\
\hline
\end{tabular}


$\mathrm{C} 39-\mathrm{C} 40$
$\mathrm{C} 39-\mathrm{H} 39$
$\mathrm{C} 40-\mathrm{C} 41$
$\mathrm{C} 40-\mathrm{H} 40$
$\mathrm{C} 41-\mathrm{C} 42$
$\mathrm{C} 41-\mathrm{H} 4$
$\mathrm{C} 43-\mathrm{N} 7$
$\mathrm{C} 43-\mathrm{C} 44$
$\mathrm{C} 43-\mathrm{C} 56$
$\mathrm{C} 44-\mathrm{N} 8$
$\mathrm{C} 44-\mathrm{C} 45$
$\mathrm{C} 45-\mathrm{C} 46$
$\mathrm{C} 45-\mathrm{C} 50$
$\mathrm{C} 46-\mathrm{C} 47$
$\mathrm{C} 46-\mathrm{H} 46$
$\mathrm{C} 47-\mathrm{C} 48$
$\mathrm{C} 47-\mathrm{H} 47$
$\mathrm{C} 48-\mathrm{C} 49$
$\mathrm{C} 48-\mathrm{H} 48$
$\mathrm{C} 49-\mathrm{C} 50$
$\mathrm{C} 49-\mathrm{H} 49$
$\mathrm{C} 50-\mathrm{C} 51$
$\mathrm{C} 51-\mathrm{C} 56$
$\mathrm{C} 51-\mathrm{C} 52$
$\mathrm{C} 52-\mathrm{C} 53$
$\mathrm{C} 52-\mathrm{H} 52$
$\mathrm{C} 53-\mathrm{C} 54$
$\mathrm{C} 53-\mathrm{H} 53$
$\mathrm{C} 54-\mathrm{C} 55$
$\mathrm{C} 54-\mathrm{H} 54$
$\mathrm{C} 55-\mathrm{C} 56$

$\mathrm{N} 1-\mathrm{C} 1-\mathrm{C} 14$

$\mathrm{N} 1-\mathrm{C} 1-\mathrm{C} 2$

$\mathrm{C} 14-\mathrm{C} 1-\mathrm{C} 2$

$\mathrm{N} 2-\mathrm{C} 2-\mathrm{C} 3$

$\mathrm{N} 2-\mathrm{C} 2-\mathrm{C} 1$

$\mathrm{C} 3-\mathrm{C} 2-\mathrm{C} 1$

$\mathrm{C} 4-\mathrm{C} 3-\mathrm{C} 8$

$\mathrm{C} 4-\mathrm{C} 3-\mathrm{C} 2$

$\mathrm{C} 8-\mathrm{C} 3-\mathrm{C} 2$

$\mathrm{C} 5-\mathrm{C} 4-\mathrm{C} 3$

$\mathrm{C} 5-\mathrm{C} 4-\mathrm{H} 4$

$\mathrm{C} 3-\mathrm{C} 4-\mathrm{H} 4$

$\mathrm{C} 4-\mathrm{C} 5-\mathrm{C} 6$

$\mathrm{C} 4-\mathrm{C} 5-\mathrm{H} 5$

$\mathrm{C} 6-\mathrm{C} 5-\mathrm{H} 5$

$\mathrm{C} 7-\mathrm{C} 6-\mathrm{C} 5$
$1.370(6)$

0.9300

$1.382(5)$

0.9300

$1.401(5)$

0.9300

$1.311(4)$

$1.457(5)$

$1.465(5)$

$1.325(4)$

$1.463(5)$

$1.399(5)$

$1.425(5)$

$1.381(5)$

0.9300

$1.383(6)$

0.9300

$1.381(7)$

0.9300

1.399 (5)

0.9300

1.465 (6)

$1.411(5)$

$1.416(5)$

1.359 (6)

0.9300

1.378 (6)

0.9300

1.389 (5)

0.9300

$1.402(5)$

$127.3(3)$

112.3 (3)

120.4 (3)

127.7 (3)

111.7 (3)

120.3 (3)

119.3 (3)

122.9 (3)

117.5 (3)

120.8 (3)

119.6

119.6

120.1 (3)

120.0

120.0

120.0 (3)
$\mathrm{N} 1-\mathrm{O} 1$

$\mathrm{N} 1-\mathrm{Ni1}$

$\mathrm{N} 2-\mathrm{O} 2$

N2-Ni1

N3-O3

$\mathrm{N} 3-\mathrm{Ni} 2$

$\mathrm{N} 4-\mathrm{O} 4$

$\mathrm{N} 5-\mathrm{O} 5$

$\mathrm{N} 5-\mathrm{Ni} 1$

$\mathrm{N} 6-\mathrm{O} 6$

$\mathrm{N} 7-\mathrm{O} 7$

$\mathrm{N} 7-\mathrm{Ni} 2$

$\mathrm{N} 8-\mathrm{O} 8$

$\mathrm{N} 8-\mathrm{Ni} 2$

N9-O9

N9- $-\mathrm{Ni} 3$

$\mathrm{N} 10-\mathrm{O} 10$

N10-Ni3

O1-H1A

$\mathrm{O} 2-\mathrm{Ni3}$

$\mathrm{O} 3-\mathrm{H} 2 \mathrm{~A}$

$\mathrm{O} 4-\mathrm{Ni} 2$

$\mathrm{O} 4-\mathrm{Ni3}$

O5-H1A

O6-Ni1

$\mathrm{O} 6-\mathrm{Ni3}$

$\mathrm{O} 7-\mathrm{H} 2 \mathrm{~A}$

O8-Ni3

O9- $\mathrm{H} 3 \mathrm{~A}$

$\mathrm{O} 10-\mathrm{H} 4 \mathrm{~A}$

C60-C59-C58

$\mathrm{C} 64-\mathrm{C} 59-\mathrm{C} 58$

C61- C60-C59

$\mathrm{C} 61-\mathrm{C} 60-\mathrm{H} 60$

$\mathrm{C} 59-\mathrm{C} 60-\mathrm{H} 60$

C62-C61- 60

C62-C61-H61

C60-C61-H61

C61-C62-C63

C61-C62-H62

C63-C62-H62

C62-C63-C64

C62-C63-H63

C64-C63-H63

C63-C64-C59

C63-C64-C65
1.349 (3)

$1.859(3)$

$1.333(3)$

1.884 (3)

1.341 (3)

$1.892(3)$

$1.363(3)$

$1.342(3)$

$1.900(3)$

$1.364(3)$

$1.344(4)$

$1.871(3)$

$1.333(4)$

$1.888(3)$

$1.365(4)$

$2.035(3)$

$1.388(4)$

$2.043(3)$

$1.29(6)$

$2.050(2)$

$1.17(5)$

$1.811(2)$

$2.044(2)$

$1.13(6)$

1.813 (2)

2.042 (2)

1.24 (6)

2.047 (2)

0.85 (6)

1.04 (6)

$123.0(3)$

$118.0(3)$

121.1 (4)

119.5

119.5

120.4 (4)

119.8

119.8

119.8 (4)

120.1

120.1

121.7 (4)

119.2

119.2

118.2 (3)

$120.5(3)$ 
C7- 6 6- 66

$\mathrm{C} 5-\mathrm{C} 6-\mathrm{H} 6$

$\mathrm{C} 6-\mathrm{C} 7-\mathrm{C} 8$

$\mathrm{C} 6-\mathrm{C} 7-\mathrm{H} 7$

$\mathrm{C} 8-\mathrm{C} 7-\mathrm{H} 7$

$\mathrm{C} 7-\mathrm{C} 8-\mathrm{C} 3$

$\mathrm{C} 7-\mathrm{C} 8-\mathrm{C} 9$

$\mathrm{C} 3-\mathrm{C} 8-\mathrm{C} 9$

$\mathrm{C} 10-\mathrm{C} 9-\mathrm{C} 14$

$\mathrm{C} 10-\mathrm{C} 9-\mathrm{C} 8$

$\mathrm{C} 14-\mathrm{C} 9-\mathrm{C} 8$

$\mathrm{C} 11-\mathrm{C} 10-\mathrm{C} 9$

$\mathrm{C} 11-\mathrm{C} 10-\mathrm{H} 10$

C9- $\mathrm{C} 10-\mathrm{H} 10$

$\mathrm{C} 10-\mathrm{C} 11-\mathrm{C} 12$

$\mathrm{C} 10-\mathrm{C} 11-\mathrm{H} 11$

$\mathrm{C} 12-\mathrm{C} 11-\mathrm{H} 11$

$\mathrm{C} 13-\mathrm{C} 12-\mathrm{C} 11$

$\mathrm{C} 13-\mathrm{C} 12-\mathrm{H} 12$

$\mathrm{C} 11-\mathrm{C} 12-\mathrm{H} 12$

$\mathrm{C} 12-\mathrm{C} 13-\mathrm{C} 14$

$\mathrm{C} 12-\mathrm{C} 13-\mathrm{H} 13$

$\mathrm{C} 14-\mathrm{C} 13-\mathrm{H} 13$

$\mathrm{C} 13-\mathrm{C} 14-\mathrm{C} 9$

$\mathrm{C} 13-\mathrm{C} 14-\mathrm{C} 1$

C9- $\mathrm{C} 14-\mathrm{C} 1$

N3- $15-\mathrm{C} 16$

N3-C15-C28

$\mathrm{C} 16-\mathrm{C} 15-\mathrm{C} 28$

$\mathrm{N} 4-\mathrm{C} 16-\mathrm{C} 15$

$\mathrm{N} 4-\mathrm{C} 16-\mathrm{C} 17$

$\mathrm{C} 15-\mathrm{C} 16-\mathrm{C} 17$

$\mathrm{C} 22-\mathrm{C} 17-\mathrm{C} 18$

$\mathrm{C} 22-\mathrm{C} 17-\mathrm{C} 16$

$\mathrm{C} 18-\mathrm{C} 17-\mathrm{C} 16$

$\mathrm{C} 19-\mathrm{C} 18-\mathrm{C} 17$

C19-C18-H18

C17-C18-H18

C18-C19-C20

C18-C19-H19

$\mathrm{C} 20-\mathrm{C} 19-\mathrm{H} 19$

$\mathrm{C} 21-\mathrm{C} 20-\mathrm{C} 19$

$\mathrm{C} 21-\mathrm{C} 20-\mathrm{H} 20$

$\mathrm{C} 19-\mathrm{C} 20-\mathrm{H} 20$

$\mathrm{C} 20-\mathrm{C} 21-\mathrm{C} 22$

$\mathrm{C} 20-\mathrm{C} 21-\mathrm{H} 21$

$\mathrm{C} 22-\mathrm{C} 21-\mathrm{H} 21$

$\mathrm{C} 17-\mathrm{C} 22-\mathrm{C} 21$
120.0

120.0

122.1 (4)

119.0

119.0

$117.6(3)$

$121.2(3)$

$121.2(3)$

$117.7(3)$

$121.0(3)$

$121.3(3)$

$122.2(3)$

118.9

118.9

$120.0(3)$

120.0

120.0

119.5 (3)

120.2

120.2

$121.1(3)$

119.4

119.4

$119.4(3)$

$122.9(3)$

$117.6(3)$

$118.6(3)$

$124.4(3)$

$117.0(3)$

$126.2(3)$

$115.3(3)$

$118.5(3)$

$120.3(3)$

$119.6(3)$

$120.1(3)$

120.7 (3)

119.6

119.6

$119.5(3)$

120.3

120.3

$120.2(3)$

119.9

119.9

121.7 (3)

119.2

119.2

$117.7(3)$
$\mathrm{C} 59-\mathrm{C} 64-\mathrm{C} 65$

C66-C65-C70

C66-C65-C64

C70-C65-C64

C67-C66- 655

C67-C66-H66

C65-C66-H66

C66-C67-C68

C66-C67-H67

C68-C67-H67

C69- $668-\mathrm{C} 67$

$\mathrm{C} 69-\mathrm{C} 68-\mathrm{H} 68$

$\mathrm{C} 67-\mathrm{C} 68-\mathrm{H} 68$

$\mathrm{C} 68-\mathrm{C} 69-\mathrm{C} 70$

C68-C69-H69

C70- $699-\mathrm{H} 69$

C65-C70-C69

C65-C70-C57

C69-C $70-\mathrm{C} 57$

C72-C71-H71A

C72-C71-H71B

H71A-C71-H71B

C72-C71- $\mathrm{H} 71 \mathrm{C}$

$\mathrm{H} 71 \mathrm{~A}-\mathrm{C} 71-\mathrm{H} 71 \mathrm{C}$

$\mathrm{H} 71 \mathrm{~B}-\mathrm{C} 71-\mathrm{H} 71 \mathrm{C}$

C73-C72-C77

$\mathrm{C} 73-\mathrm{C} 72-\mathrm{C} 71$

$\mathrm{C} 77-\mathrm{C} 72-\mathrm{C} 71$

$\mathrm{C} 74-\mathrm{C} 73-\mathrm{C} 72$

$\mathrm{C} 74-\mathrm{C} 73-\mathrm{H} 73$

$\mathrm{C} 72-\mathrm{C} 73-\mathrm{H} 73$

$\mathrm{C} 73-\mathrm{C} 74-\mathrm{C} 75$

C73-C74-H74

C75-C74-H74

C76-C75-C74

$\mathrm{C} 76-\mathrm{C} 75-\mathrm{H} 75$

C74-C75-H75

C75-C76-C77

C75-C76-H76

C77-C76-H76

C76-C77-C72

C76- C77-H77

C72-C77-H77

C79-C $78-\mathrm{H} 78 \mathrm{~A}$

C79-C78-H78B

$\mathrm{H} 78 \mathrm{~A}-\mathrm{C} 78-\mathrm{H} 78 \mathrm{~B}$

C79-C $78-\mathrm{H} 78 \mathrm{C}$

$\mathrm{H} 78 \mathrm{~A}-\mathrm{C} 78-\mathrm{H} 78 \mathrm{C}$
$121.3(3)$

$118.8(3)$

$120.2(3)$

$120.9(3)$

$121.6(4)$

119.2

119.2

119.8 (4)

120.1

120.1

$121.3(4)$

119.3

119.3

119.7 (3)

120.1

120.1

118.7 (3)

$118.0(3)$

123.3 (3)

109.5

109.5

109.5

109.5

109.5

109.5

117.9 (4)

$121.0(5)$

$121.2(5)$

120.7 (4)

119.7

119.7

120.6 (4)

119.7

119.7

119.6 (4)

120.2

120.2

119.9 (4)

120.0

120.0

121.3 (4)

119.3

119.3

109.5

109.5

109.5

109.5

109.5 


\begin{tabular}{|c|c|}
\hline $\mathrm{C} 17-\mathrm{C} 22-\mathrm{C} 23$ & $120.3(3)$ \\
\hline $\mathrm{C} 21-\mathrm{C} 22-\mathrm{C} 23$ & $122.0(3)$ \\
\hline $\mathrm{C} 24-\mathrm{C} 23-\mathrm{C} 28$ & $117.8(3)$ \\
\hline $\mathrm{C} 24-\mathrm{C} 23-\mathrm{C} 22$ & $121.4(3)$ \\
\hline $\mathrm{C} 28-\mathrm{C} 23-\mathrm{C} 22$ & $120.7(3)$ \\
\hline $\mathrm{C} 25-\mathrm{C} 24-\mathrm{C} 23$ & $122.4(3)$ \\
\hline $\mathrm{C} 25-\mathrm{C} 24-\mathrm{H} 24$ & 118.8 \\
\hline $\mathrm{C} 23-\mathrm{C} 24-\mathrm{H} 24$ & 118.8 \\
\hline $\mathrm{C} 24-\mathrm{C} 25-\mathrm{C} 26$ & $119.6(3)$ \\
\hline $\mathrm{C} 24-\mathrm{C} 25-\mathrm{H} 25$ & 120.2 \\
\hline $\mathrm{C} 26-\mathrm{C} 25-\mathrm{H} 25$ & 120.2 \\
\hline $\mathrm{C} 25-\mathrm{C} 26-\mathrm{C} 27$ & $119.9(4)$ \\
\hline $\mathrm{C} 25-\mathrm{C} 26-\mathrm{H} 26$ & 120.0 \\
\hline $\mathrm{C} 27-\mathrm{C} 26-\mathrm{H} 26$ & 120.0 \\
\hline $\mathrm{C} 26-\mathrm{C} 27-\mathrm{C} 28$ & $121.5(3)$ \\
\hline $\mathrm{C} 26-\mathrm{C} 27-\mathrm{H} 27$ & 119.3 \\
\hline $\mathrm{C} 28-\mathrm{C} 27-\mathrm{H} 27$ & 119.3 \\
\hline $\mathrm{C} 27-\mathrm{C} 28-\mathrm{C} 23$ & $118.6(3)$ \\
\hline $\mathrm{C} 27-\mathrm{C} 28-\mathrm{C} 15$ & $122.4(3)$ \\
\hline $\mathrm{C} 23-\mathrm{C} 28-\mathrm{C} 15$ & $118.9(3)$ \\
\hline $\mathrm{N} 5-\mathrm{C} 29-\mathrm{C} 30$ & $117.8(3)$ \\
\hline $\mathrm{N} 5-\mathrm{C} 29-\mathrm{C} 42$ & $125.7(3)$ \\
\hline $\mathrm{C} 30-\mathrm{C} 29-\mathrm{C} 42$ & $116.4(3)$ \\
\hline $\mathrm{N} 6-\mathrm{C} 30-\mathrm{C} 29$ & $126.8(3)$ \\
\hline $\mathrm{N} 6-\mathrm{C} 30-\mathrm{C} 31$ & $114.6(3)$ \\
\hline $\mathrm{C} 29-\mathrm{C} 30-\mathrm{C} 31$ & $118.6(3)$ \\
\hline $\mathrm{C} 32-\mathrm{C} 31-\mathrm{C} 36$ & $120.4(3)$ \\
\hline $\mathrm{C} 32-\mathrm{C} 31-\mathrm{C} 30$ & $120.9(3)$ \\
\hline $\mathrm{C} 36-\mathrm{C} 31-\mathrm{C} 30$ & $118.7(3)$ \\
\hline $\mathrm{C} 33-\mathrm{C} 32-\mathrm{C} 31$ & $120.1(4)$ \\
\hline $\mathrm{C} 33-\mathrm{C} 32-\mathrm{H} 32$ & 120.0 \\
\hline $\mathrm{C} 31-\mathrm{C} 32-\mathrm{H} 32$ & 120.0 \\
\hline $\mathrm{C} 34-\mathrm{C} 33-\mathrm{C} 32$ & $120.1(4)$ \\
\hline $\mathrm{C} 34-\mathrm{C} 33-\mathrm{H} 33$ & 119.9 \\
\hline $\mathrm{C} 32-\mathrm{C} 33-\mathrm{H} 33$ & 119.9 \\
\hline C33-C34-C35 & $120.7(4)$ \\
\hline $\mathrm{C} 33-\mathrm{C} 34-\mathrm{H} 34$ & 119.7 \\
\hline $\mathrm{C} 35-\mathrm{C} 34-\mathrm{H} 34$ & 119.7 \\
\hline $\mathrm{C} 34-\mathrm{C} 35-\mathrm{C} 36$ & $121.0(4)$ \\
\hline $\mathrm{C} 34-\mathrm{C} 35-\mathrm{H} 35$ & 119.5 \\
\hline $\mathrm{C} 36-\mathrm{C} 35-\mathrm{H} 35$ & 119.5 \\
\hline $\mathrm{C} 31-\mathrm{C} 36-\mathrm{C} 35$ & $117.8(3)$ \\
\hline $\mathrm{C} 31-\mathrm{C} 36-\mathrm{C} 37$ & $119.5(3)$ \\
\hline $\mathrm{C} 35-\mathrm{C} 36-\mathrm{C} 37$ & $122.8(3)$ \\
\hline $\mathrm{C} 38-\mathrm{C} 37-\mathrm{C} 42$ & $118.0(3)$ \\
\hline $\mathrm{C} 38-\mathrm{C} 37-\mathrm{C} 36$ & $120.8(3)$ \\
\hline $\mathrm{C} 42-\mathrm{C} 37-\mathrm{C} 36$ & $121.1(3)$ \\
\hline & \\
\hline
\end{tabular}

$\begin{array}{ll}\mathrm{H} 78 \mathrm{~B}-\mathrm{C} 78-\mathrm{H} 78 \mathrm{C} & 109.5 \\ \mathrm{C} 80-\mathrm{C} 79-\mathrm{C} 84 & 119.1(5) \\ \mathrm{C} 80-\mathrm{C} 79-\mathrm{C} 78 & 121.0(5) \\ \mathrm{C} 84-\mathrm{C} 79-\mathrm{C} 78 & 119.9(5) \\ \mathrm{C} 81-\mathrm{C} 80-\mathrm{C} 79 & 121.2(5) \\ \mathrm{C} 81-\mathrm{C} 80-\mathrm{H} 80 & 119.4 \\ \mathrm{C} 79-\mathrm{C} 80-\mathrm{H} 80 & 119.4 \\ \mathrm{C} 80-\mathrm{C} 81-\mathrm{C} 82 & 119.5(5) \\ \mathrm{C} 80-\mathrm{C} 81-\mathrm{H} 81 & 120.2 \\ \mathrm{C} 82-\mathrm{C} 81-\mathrm{H} 81 & 120.2 \\ \mathrm{C} 83-\mathrm{C} 82-\mathrm{C} 81 & 119.6(5) \\ \mathrm{C} 83-\mathrm{C} 82-\mathrm{H} 82 & 120.2 \\ \mathrm{C} 81-\mathrm{C} 82-\mathrm{H} 82 & 120.2 \\ \mathrm{C} 84-\mathrm{C} 83-\mathrm{C} 82 & 121.0(5) \\ \mathrm{C} 84-\mathrm{C} 83-\mathrm{H} 83 & 119.5\end{array}$

$\mathrm{C} 84-\mathrm{C} 83-\mathrm{H} 83 \quad 119.5$

$\mathrm{C} 82-\mathrm{C} 83-\mathrm{H} 83-119.5$

$\mathrm{C} 83-\mathrm{C} 84-\mathrm{C} 79 \quad 119.5$ (5)

$\mathrm{C} 83-\mathrm{C} 84-\mathrm{H} 84 \quad 120.2$

$\mathrm{C} 79-\mathrm{C} 84-\mathrm{H} 84 \quad 120.2$

C79A-C78A-H78D $\quad 109.5$

$\mathrm{C} 79 \mathrm{~A}-\mathrm{C} 78 \mathrm{~A}-\mathrm{H} 78 \mathrm{E} \quad 109.5$

$\mathrm{H} 78 \mathrm{D}-\mathrm{C} 78 \mathrm{~A}-\mathrm{H} 78 \mathrm{E} \quad 109.5$

$\mathrm{C} 79 \mathrm{~A}-\mathrm{C} 78 \mathrm{~A}-\mathrm{H} 78 \mathrm{~F} \quad 109.5$

$\mathrm{H} 78 \mathrm{D}-\mathrm{C} 78 \mathrm{~A}-\mathrm{H} 78 \mathrm{~F} \quad 109.5$

$\mathrm{H} 78 \mathrm{E}-\mathrm{C} 78 \mathrm{~A}-\mathrm{H} 78 \mathrm{~F} \quad 109.5$

$\mathrm{C} 84 \mathrm{~A}-\mathrm{C} 79 \mathrm{~A}-\mathrm{C} 80 \mathrm{~A} \quad 120.4$ (17)

$\mathrm{C} 84 \mathrm{~A}-\mathrm{C} 79 \mathrm{~A}-\mathrm{C} 78 \mathrm{~A} \quad 121.6(11)$

$\mathrm{C} 80 \mathrm{~A}-\mathrm{C} 79 \mathrm{~A}-\mathrm{C} 78 \mathrm{~A} \quad 117.9(12)$

$\mathrm{C} 82 \mathrm{~A}-\mathrm{C} 81 \mathrm{~A}-\mathrm{C} 80 \mathrm{~A} \quad 123.7$ (16)

$\mathrm{C} 82 \mathrm{~A}-\mathrm{C} 81 \mathrm{~A}-\mathrm{H} 81 \mathrm{~A} \quad 118.2$

$\mathrm{C} 80 \mathrm{~A}-\mathrm{C} 81 \mathrm{~A}-\mathrm{H} 81 \mathrm{~A}$

$\mathrm{C} 79 \mathrm{~A}-\mathrm{C} 80 \mathrm{~A}-\mathrm{C} 81 \mathrm{~A}$

$\mathrm{C} 79 \mathrm{~A}-\mathrm{C} 80 \mathrm{~A}-\mathrm{H} 80 \mathrm{~A} \quad 122.1$

$\mathrm{C} 81 \mathrm{~A}-\mathrm{C} 80 \mathrm{~A}-\mathrm{H} 80 \mathrm{~A}$

$\mathrm{C} 81 \mathrm{~A}-\mathrm{C} 82 \mathrm{~A}-\mathrm{C} 83 \mathrm{~A} \quad 121$ (2)

$\mathrm{C} 81 \mathrm{~A}-\mathrm{C} 82 \mathrm{~A}-\mathrm{H} 82 \mathrm{~A} \quad 119.5$

$\mathrm{C} 83 \mathrm{~A}-\mathrm{C} 82 \mathrm{~A}-\mathrm{H} 82 \mathrm{~A} \quad 119.5$

$\mathrm{C} 82 \mathrm{~A}-\mathrm{C} 83 \mathrm{~A}-\mathrm{C} 84 \mathrm{~A} \quad 117.8$ (17)

$\mathrm{C} 82 \mathrm{~A}-\mathrm{C} 83 \mathrm{~A}-\mathrm{H} 83 \mathrm{~A} \quad 121.1$

$\mathrm{C} 84 \mathrm{~A}-\mathrm{C} 83 \mathrm{~A}-\mathrm{H} 83 \mathrm{~A} \quad 121.1$

$\mathrm{C} 79 \mathrm{~A}-\mathrm{C} 84 \mathrm{~A}-\mathrm{C} 83 \mathrm{~A}$

C79A-C84A-H84A 119.3

$\mathrm{C} 83 \mathrm{~A}-\mathrm{C} 84 \mathrm{~A}-\mathrm{H} 84 \mathrm{~A} \quad 119.3$

C1-N1-O1 120.2 (3)

$\mathrm{C} 1-\mathrm{N} 1-\mathrm{Ni1} \quad 117.4(2)$

$\mathrm{O} 1-\mathrm{N} 1-\mathrm{Ni1} \quad 122.4(2)$

$\mathrm{C} 2-\mathrm{N} 2-\mathrm{O} 2$

$116.3(2)$ 
C39- $338-\mathrm{H} 38$

C37-C $38-\mathrm{H} 38$

$\mathrm{C} 40-\mathrm{C} 39-\mathrm{C} 38$

C40-C39- H39

C38-C39-H39

C39-C40-C41

$\mathrm{C} 39-\mathrm{C} 40-\mathrm{H} 40$

$\mathrm{C} 41-\mathrm{C} 40-\mathrm{H} 40$

$\mathrm{C} 40-\mathrm{C} 41-\mathrm{C} 42$

$\mathrm{C} 40-\mathrm{C} 41-\mathrm{H} 41$

$\mathrm{C} 42-\mathrm{C} 41-\mathrm{H} 41$

$\mathrm{C} 41-\mathrm{C} 42-\mathrm{C} 37$

$\mathrm{C} 41-\mathrm{C} 42-\mathrm{C} 29$

C37-C $42-\mathrm{C} 29$

N7-C43-C44

$\mathrm{N} 7-\mathrm{C} 43-\mathrm{C} 56$

$\mathrm{C} 44-\mathrm{C} 43-\mathrm{C} 56$

$\mathrm{N} 8-\mathrm{C} 44-\mathrm{C} 43$

$\mathrm{N} 8-\mathrm{C} 44-\mathrm{C} 45$

$\mathrm{C} 43-\mathrm{C} 44-\mathrm{C} 45$

$\mathrm{C} 46-\mathrm{C} 45-\mathrm{C} 50$

$\mathrm{C} 46-\mathrm{C} 45-\mathrm{C} 44$

$\mathrm{C} 50-\mathrm{C} 45-\mathrm{C} 44$

$\mathrm{C} 47-\mathrm{C} 46-\mathrm{C} 45$

$\mathrm{C} 47-\mathrm{C} 46-\mathrm{H} 46$

$\mathrm{C} 45-\mathrm{C} 46-\mathrm{H} 46$

$\mathrm{C} 46-\mathrm{C} 47-\mathrm{C} 48$

$\mathrm{C} 46-\mathrm{C} 47-\mathrm{H} 47$

$\mathrm{C} 48-\mathrm{C} 47-\mathrm{H} 47$

$\mathrm{C} 49-\mathrm{C} 48-\mathrm{C} 47$

$\mathrm{C} 49-\mathrm{C} 48-\mathrm{H} 48$

$\mathrm{C} 47-\mathrm{C} 48-\mathrm{H} 48$

$\mathrm{C} 48-\mathrm{C} 49-\mathrm{C} 50$

$\mathrm{C} 48-\mathrm{C} 49-\mathrm{H} 49$

$\mathrm{C} 50-\mathrm{C} 49-\mathrm{H} 49$

$\mathrm{C} 49-\mathrm{C} 50-\mathrm{C} 45$

C49-C50-C51

$\mathrm{C} 45-\mathrm{C} 50-\mathrm{C} 51$

C56-C51-C52

C56-C51-C50

C52-C51-C50

$\mathrm{C} 53-\mathrm{C} 52-\mathrm{C} 51$

$\mathrm{C} 53-\mathrm{C} 52-\mathrm{H} 52$

$\mathrm{C} 51-\mathrm{C} 52-\mathrm{H} 52$

$\mathrm{C} 52-\mathrm{C} 53-\mathrm{C} 54$

$\mathrm{C} 52-\mathrm{C} 53-\mathrm{H} 53$

C54-C53-H53

C53-C54-C55
119.1

119.1

120.0 (4)

120.0

120.0

$120.2(4)$

119.9

119.9

$121.2(4)$

119.4

119.4

118.7 (3)

$122.4(3)$

$118.6(3)$

112.4 (3)

$127.1(3)$

$120.4(3)$

$112.2(3)$

$127.3(3)$

120.4 (3)

$120.2(3)$

122.5 (3)

$117.1(3)$

120.6 (4)

119.7

119.7

119.8 (4)

120.1

120.1

120.4 (4)

119.8

119.8

121.9 (4)

119.0

119.0

117.1 (4)

121.4 (4)

$121.5(3)$

117.1 (4)

$121.7(3)$

$121.2(4)$

122.3 (4)

118.9

118.9

120.6 (4)

119.7

119.7

119.4 (4)
$\mathrm{O} 2-\mathrm{N} 2-\mathrm{Ni} 1$

$\mathrm{C} 15-\mathrm{N} 3-\mathrm{O} 3$

$\mathrm{C} 15-\mathrm{N} 3-\mathrm{Ni} 2$

$\mathrm{O} 3-\mathrm{N} 3-\mathrm{Ni} 2$

$\mathrm{C} 16-\mathrm{N} 4-\mathrm{O} 4$

C29-N5-O5

C29-N5-Ni1

O5-N5-Ni1

C30-N6-O6

C43-N7-O7

$\mathrm{C} 43-\mathrm{N} 7-\mathrm{Ni} 2$

$\mathrm{O} 7-\mathrm{N} 7-\mathrm{Ni} 2$

C44-N8-O8

$\mathrm{C} 44-\mathrm{N} 8-\mathrm{Ni} 2$

$\mathrm{O} 8-\mathrm{N} 8-\mathrm{Ni} 2$

C57-N9-O9

C57-N9-Ni3

O9-N9-Ni3

$\mathrm{C} 58-\mathrm{N} 10-\mathrm{O} 10$

C58-N10-Ni3

$\mathrm{O} 10-\mathrm{N} 10-\mathrm{Ni} 3$

$\mathrm{N} 1-\mathrm{O} 1-\mathrm{H} 1 \mathrm{~A}$

$\mathrm{N} 2-\mathrm{O} 2-\mathrm{Ni} 3$

$\mathrm{N} 3-\mathrm{O} 3-\mathrm{H} 2 \mathrm{~A}$

$\mathrm{N} 4-\mathrm{O} 4-\mathrm{Ni} 2$

$\mathrm{N} 4-\mathrm{O} 4-\mathrm{Ni} 3$

$\mathrm{Ni} 2-\mathrm{O} 4-\mathrm{Ni} 3$

N5-O5-H1A

N6-O6-Ni1

$\mathrm{N} 6-\mathrm{O} 6-\mathrm{Ni} 3$

$\mathrm{Ni1}-\mathrm{O} 6-\mathrm{Ni3}$

$\mathrm{N} 7-\mathrm{O} 7-\mathrm{H} 2 \mathrm{~A}$

$\mathrm{N} 8-\mathrm{O} 8-\mathrm{Ni} 3$

N9-O9- 33 A

$\mathrm{N} 10-\mathrm{O} 10-\mathrm{H} 4 \mathrm{~A}$

O6-Ni1-N1

O6- Ni1-N2

$\mathrm{N} 1-\mathrm{Ni} 1-\mathrm{N} 2$

O6-Ni1-N5

N1-Ni1-N5

N2-Ni1-N5

$\mathrm{O} 4-\mathrm{Ni} 2-\mathrm{N} 7$

$\mathrm{O} 4-\mathrm{Ni} 2-\mathrm{N} 8$

$\mathrm{N} 7-\mathrm{Ni} 2-\mathrm{N} 8$

$\mathrm{O} 4-\mathrm{Ni} 2-\mathrm{N} 3$

$\mathrm{N} 7-\mathrm{Ni} 2-\mathrm{N} 3$

$\mathrm{N} 8-\mathrm{Ni} 2-\mathrm{N} 3$

N9-Ni3-O6
$122.3(2)$

116.8 (3)

$126.6(2)$

116.3 (2)

$119.1(3)$

116.3 (3)

$126.6(2)$

116.3 (2)

$118.6(3)$

$120.8(3)$

117.0 (2)

$122.0(2)$

121.7 (3)

115.9 (2)

$122.3(2)$

117.4 (3)

$118.8(2)$

$122.5(2)$

117.1 (3)

118.1 (2)

$123.1(2)$

101 (3)

111.88 (18)

103 (2)

127.34 (19)

116.88 (18)

115.78 (11)

107 (3)

127.55 (19)

116.43 (18)

115.93 (11)

98 (2)

111.97 (18)

93 (4)

102 (3)

169.58 (11)

87.73 (10)

81.94 (11)

91.03 (11)

$99.21(12)$

176.81 (12)

$170.22(11)$

88.31 (11)

81.93 (12)

90.94 (11)

98.79 (12)

177.16 (12)

165.53 (11) 


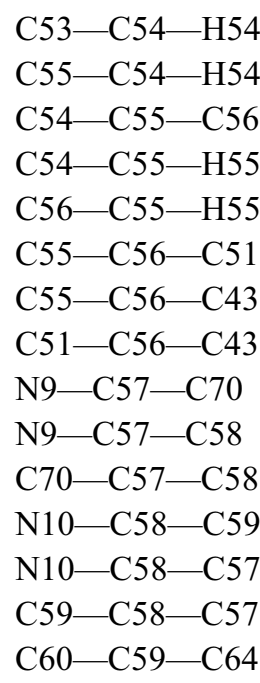

$\mathrm{N} 1-\mathrm{C} 1-\mathrm{C} 2-\mathrm{N} 2$

$\mathrm{C} 14-\mathrm{C} 1-\mathrm{C} 2-\mathrm{N} 2$

$\mathrm{N} 1-\mathrm{C} 1-\mathrm{C} 2-\mathrm{C} 3$

$\mathrm{C} 14-\mathrm{C} 1-\mathrm{C} 2-\mathrm{C} 3$

$\mathrm{N} 2-\mathrm{C} 2-\mathrm{C} 3-\mathrm{C} 4$

$\mathrm{C} 1-\mathrm{C} 2-\mathrm{C} 3-\mathrm{C} 4$

$\mathrm{N} 2-\mathrm{C} 2-\mathrm{C} 3-\mathrm{C} 8$

$\mathrm{C} 1-\mathrm{C} 2-\mathrm{C} 3-\mathrm{C} 8$

$\mathrm{C} 8-\mathrm{C} 3-\mathrm{C} 4-\mathrm{C} 5$

$\mathrm{C} 2-\mathrm{C} 3-\mathrm{C} 4-\mathrm{C} 5$

$\mathrm{C} 3-\mathrm{C} 4-\mathrm{C} 5-\mathrm{C} 6$

$\mathrm{C} 4-\mathrm{C} 5-\mathrm{C} 6-\mathrm{C} 7$

$\mathrm{C} 5-\mathrm{C} 6-\mathrm{C} 7-\mathrm{C} 8$

$\mathrm{C} 6-\mathrm{C} 7-\mathrm{C} 8-\mathrm{C} 3$

$\mathrm{C} 6-\mathrm{C} 7-\mathrm{C} 8-\mathrm{C} 9$

$\mathrm{C} 4-\mathrm{C} 3-\mathrm{C} 8-\mathrm{C} 7$

$\mathrm{C} 2-\mathrm{C} 3-\mathrm{C} 8-\mathrm{C} 7$

$\mathrm{C} 4-\mathrm{C} 3-\mathrm{C} 8-\mathrm{C} 9$

$\mathrm{C} 2-\mathrm{C} 3-\mathrm{C} 8-\mathrm{C} 9$

$\mathrm{C} 7-\mathrm{C} 8-\mathrm{C} 9-\mathrm{C} 10$

$\mathrm{C} 3-\mathrm{C} 8-\mathrm{C} 9-\mathrm{C} 10$

$\mathrm{C} 7-\mathrm{C} 8-\mathrm{C} 9-\mathrm{C} 14$

$\mathrm{C} 3-\mathrm{C} 8-\mathrm{C} 9-\mathrm{C} 14$

$\mathrm{C} 14-\mathrm{C} 9-\mathrm{C} 10-\mathrm{C} 11$

$\mathrm{C} 8-\mathrm{C} 9-\mathrm{C} 10-\mathrm{C} 11$

C9- $\mathrm{C} 10-\mathrm{C} 11-\mathrm{C} 12$

$\mathrm{C} 10-\mathrm{C} 11-\mathrm{C} 12-\mathrm{C} 13$

$\mathrm{C} 11-\mathrm{C} 12-\mathrm{C} 13-\mathrm{C} 14$

$\mathrm{C} 12-\mathrm{C} 13-\mathrm{C} 14-\mathrm{C} 9$

$\mathrm{C} 12-\mathrm{C} 13-\mathrm{C} 14-\mathrm{C} 1$

$\mathrm{C} 10-\mathrm{C} 9-\mathrm{C} 14-\mathrm{C} 13$

$\mathrm{C} 8-\mathrm{C} 9-\mathrm{C} 14-\mathrm{C} 13$
120.3

120.3

120.9 (4)

119.5

119.5

119.8 (3)

$123.0(3)$

117.3 (3)

$128.8(3)$

$112.0(3)$

119.2 (3)

$128.4(3)$

$112.7(3)$

118.8 (3)

$118.8(3)$

6.8 (4)

$-174.0(3)$

-166.9 (3)

12.4 (5)

-0.7 (5)

171.9 (3)

$-174.4(3)$

$-1.8(4)$

$3.3(5)$

$-170.3(3)$

$-0.6(5)$

$-1.8(6)$

$1.4(6)$

$1.3(5)$

$-179.2(3)$

$-3.6(5)$

170.4 (3)

176.9 (3)

$-9.1(4)$

$13.2(5)$

$-167.3(3)$

-169.4 (3)

$10.1(5)$

$1.0(5)$

$178.6(3)$

$-1.6(6)$

$0.8(5)$

$0.6(5)$

$-1.2(5)$

-178.7 (3)

0.4 (5)

$-177.2(3)$
N9-Ni3-N10

O6- $-\mathrm{Ni3}-\mathrm{N} 10$

$\mathrm{N} 9-\mathrm{Ni3}-\mathrm{O} 4$

$\mathrm{O} 6-\mathrm{Ni} 3-\mathrm{O} 4$

$\mathrm{N} 10-\mathrm{Ni3}-\mathrm{O} 4$

$\mathrm{N} 9-\mathrm{Ni3}-\mathrm{O} 8$

$\mathrm{O} 6-\mathrm{Ni} 3-\mathrm{O} 8$

$\mathrm{N} 10-\mathrm{Ni3}-\mathrm{O} 8$

$\mathrm{O} 4-\mathrm{Ni} 3-\mathrm{O} 8$

$\mathrm{N} 9-\mathrm{Ni} 3-\mathrm{O} 2$

$\mathrm{O} 6-\mathrm{Ni} 3-\mathrm{O} 2$

$\mathrm{N} 10-\mathrm{Ni3}-\mathrm{O} 2$

$\mathrm{O} 4-\mathrm{Ni} 3-\mathrm{O} 2$

$\mathrm{O} 8-\mathrm{Ni} 3-\mathrm{O} 2$

C50-C $51-\mathrm{C} 56-\mathrm{C} 43$

N7-C43-C56-C55

$\mathrm{C} 44-\mathrm{C} 43-\mathrm{C} 56-\mathrm{C} 55$

$\mathrm{N} 7-\mathrm{C} 43-\mathrm{C} 56-\mathrm{C} 51$

$\mathrm{C} 44-\mathrm{C} 43-\mathrm{C} 56-\mathrm{C} 51$

N9-C57-C58-N10

$\mathrm{C} 70-\mathrm{C} 57-\mathrm{C} 58-\mathrm{N} 10$

N9-C57-C58-C59

C70-C57-C58-C59

$\mathrm{N} 10-\mathrm{C} 58-\mathrm{C} 59-\mathrm{C} 60$

$\mathrm{C} 57-\mathrm{C} 58-\mathrm{C} 59-\mathrm{C} 60$

$\mathrm{N} 10-\mathrm{C} 58-\mathrm{C} 59-\mathrm{C} 64$

C57-C58-C59-C64

C64-C59-C60-C61

C58-C59-C60-C61

$\mathrm{C} 59-\mathrm{C} 60-\mathrm{C} 61-\mathrm{C} 62$

C60-C61-C62-C63

C61-C62-C63-C64

C62-C63-C64-C59

C62-C63-C64-C65

C60-C59-C64-C63

C58-C59-C64-C63

$\mathrm{C} 60-\mathrm{C} 59-\mathrm{C} 64-\mathrm{C} 65$

$\mathrm{C} 58-\mathrm{C} 59-\mathrm{C} 64-\mathrm{C} 65$

C63-C64-C65-C66

C59-C64-C65-C66

$\mathrm{C} 63-\mathrm{C} 64-\mathrm{C} 65-\mathrm{C} 70$

C59-C64-C65-C70

C70-C65-C66-C67

C64-C65-C66-C67

C65-C66-C67-C68

C66-C67-C68-C69
$76.02(11)$

$95.97(10)$

$96.00(10)$

$94.37(10)$

$164.78(11)$

$104.85(10)$

$86.82(10)$

$89.16(10)$

$80.29(9)$

$91.16(10)$

79.47 (9)

$106.60(10)$

86.25 (9)

159.99 (9)

$-0.1(5)$

$-9.7(6)$

$168.1(3)$

$171.5(3)$

$-10.7(5)$

$16.8(4)$

$-160.8(3)$

$-161.1(3)$

$21.3(4)$

$-17.7(6)$

$159.9(3)$

$166.4(3)$

$-16.0(5)$

$0.6(6)$

$-175.3(4)$

$-2.1(6)$

1.9 (7)

-0.2 (7)

$-1.3(6)$

$176.6(4)$

$1.1(5)$

$177.2(3)$

-176.9 (3)

-0.8 (5)

$13.5(5)$

$-168.6(3)$

-164.5 (3)

$13.4(5)$

3.0 (6)

-175.1 (4)

-0.1 (6)

-2.1 (6) 


\begin{tabular}{|c|c|}
\hline $\mathrm{C} 10-\mathrm{C} 9-\mathrm{C} 14-\mathrm{C} 1$ & $178.0(3)$ \\
\hline $\mathrm{C} 8-\mathrm{C} 9-\mathrm{C} 14-\mathrm{C} 1$ & $0.5(5)$ \\
\hline $\mathrm{N} 1-\mathrm{C} 1-\mathrm{C} 14-\mathrm{C} 13$ & $-14.7(5)$ \\
\hline $\mathrm{C} 2-\mathrm{C} 1-\mathrm{C} 14-\mathrm{C} 13$ & $166.2(3)$ \\
\hline $\mathrm{N} 1-\mathrm{C} 1-\mathrm{C} 14-\mathrm{C} 9$ & $167.7(3)$ \\
\hline $\mathrm{C} 2-\mathrm{C} 1-\mathrm{C} 14-\mathrm{C} 9$ & $-11.4(5)$ \\
\hline $\mathrm{N} 3-\mathrm{C} 15-\mathrm{C} 16-\mathrm{N} 4$ & $26.0(5)$ \\
\hline $\mathrm{C} 28-\mathrm{C} 15-\mathrm{C} 16-\mathrm{N} 4$ & $-154.2(3)$ \\
\hline $\mathrm{N} 3-\mathrm{C} 15-\mathrm{C} 16-\mathrm{C} 17$ & $-153.7(3)$ \\
\hline $\mathrm{C} 28-\mathrm{C} 15-\mathrm{C} 16-\mathrm{C} 17$ & $26.0(4)$ \\
\hline $\mathrm{N} 4-\mathrm{C} 16-\mathrm{C} 17-\mathrm{C} 22$ & $166.5(3)$ \\
\hline $\mathrm{C} 15-\mathrm{C} 16-\mathrm{C} 17-\mathrm{C} 22$ & $-13.8(4)$ \\
\hline $\mathrm{N} 4-\mathrm{C} 16-\mathrm{C} 17-\mathrm{C} 18$ & $-14.7(4)$ \\
\hline $\mathrm{C} 15-\mathrm{C} 16-\mathrm{C} 17-\mathrm{C} 18$ & $165.1(3)$ \\
\hline $\mathrm{C} 22-\mathrm{C} 17-\mathrm{C} 18-\mathrm{C} 19$ & $-1.0(5)$ \\
\hline $\mathrm{C} 16-\mathrm{C} 17-\mathrm{C} 18-\mathrm{C} 19$ & $-179.9(3)$ \\
\hline $\mathrm{C} 17-\mathrm{C} 18-\mathrm{C} 19-\mathrm{C} 20$ & $1.6(5)$ \\
\hline $\mathrm{C} 18-\mathrm{C} 19-\mathrm{C} 20-\mathrm{C} 21$ & $-0.4(5)$ \\
\hline $\mathrm{C} 19-\mathrm{C} 20-\mathrm{C} 21-\mathrm{C} 22$ & $-1.3(6)$ \\
\hline $\mathrm{C} 18-\mathrm{C} 17-\mathrm{C} 22-\mathrm{C} 21$ & $-0.6(5)$ \\
\hline $\mathrm{C} 16-\mathrm{C} 17-\mathrm{C} 22-\mathrm{C} 21$ & $178.2(3)$ \\
\hline $\mathrm{C} 18-\mathrm{C} 17-\mathrm{C} 22-\mathrm{C} 23$ & $176.3(3)$ \\
\hline $\mathrm{C} 16-\mathrm{C} 17-\mathrm{C} 22-\mathrm{C} 23$ & $-4.9(5)$ \\
\hline $\mathrm{C} 20-\mathrm{C} 21-\mathrm{C} 22-\mathrm{C} 17$ & $1.8(5)$ \\
\hline $\mathrm{C} 20-\mathrm{C} 21-\mathrm{C} 22-\mathrm{C} 23$ & $-175.1(3)$ \\
\hline $\mathrm{C} 17-\mathrm{C} 22-\mathrm{C} 23-\mathrm{C} 24$ & $-166.6(3)$ \\
\hline $\mathrm{C} 21-\mathrm{C} 22-\mathrm{C} 23-\mathrm{C} 24$ & $10.2(5)$ \\
\hline $\mathrm{C} 17-\mathrm{C} 22-\mathrm{C} 23-\mathrm{C} 28$ & $11.0(5)$ \\
\hline $\mathrm{C} 21-\mathrm{C} 22-\mathrm{C} 23-\mathrm{C} 28$ & $-172.2(3)$ \\
\hline $\mathrm{C} 28-\mathrm{C} 23-\mathrm{C} 24-\mathrm{C} 25$ & $-1.3(5)$ \\
\hline $\mathrm{C} 22-\mathrm{C} 23-\mathrm{C} 24-\mathrm{C} 25$ & $176.4(3)$ \\
\hline $\mathrm{C} 23-\mathrm{C} 24-\mathrm{C} 25-\mathrm{C} 26$ & $-2.0(6)$ \\
\hline $\mathrm{C} 24-\mathrm{C} 25-\mathrm{C} 26-\mathrm{C} 27$ & $2.8(6)$ \\
\hline $\mathrm{C} 25-\mathrm{C} 26-\mathrm{C} 27-\mathrm{C} 28$ & $-0.1(6)$ \\
\hline $\mathrm{C} 26-\mathrm{C} 27-\mathrm{C} 28-\mathrm{C} 23$ & $-3.3(5)$ \\
\hline $\mathrm{C} 26-\mathrm{C} 27-\mathrm{C} 28-\mathrm{C} 15$ & $-178.9(3)$ \\
\hline $\mathrm{C} 24-\mathrm{C} 23-\mathrm{C} 28-\mathrm{C} 27$ & $3.9(5)$ \\
\hline $\mathrm{C} 22-\mathrm{C} 23-\mathrm{C} 28-\mathrm{C} 27$ & $-173.8(3)$ \\
\hline $\mathrm{C} 24-\mathrm{C} 23-\mathrm{C} 28-\mathrm{C} 15$ & $179.6(3)$ \\
\hline $\mathrm{C} 22-\mathrm{C} 23-\mathrm{C} 28-\mathrm{C} 15$ & $1.9(5)$ \\
\hline $\mathrm{N} 3-\mathrm{C} 15-\mathrm{C} 28-\mathrm{C} 27$ & $-24.8(5)$ \\
\hline $\mathrm{C} 16-\mathrm{C} 15-\mathrm{C} 28-\mathrm{C} 27$ & $155.5(3)$ \\
\hline $\mathrm{N} 3-\mathrm{C} 15-\mathrm{C} 28-\mathrm{C} 23$ & $159.6(3)$ \\
\hline $\mathrm{C} 16-\mathrm{C} 15-\mathrm{C} 28-\mathrm{C} 23$ & $-20.1(4)$ \\
\hline $\mathrm{N} 5-\mathrm{C} 29-\mathrm{C} 30-\mathrm{N} 6$ & $28.4(5)$ \\
\hline $\mathrm{C} 42-\mathrm{C} 29-\mathrm{C} 30-\mathrm{N} 6$ & $-150.6(3)$ \\
\hline $\mathrm{N} 5-\mathrm{C} 29-\mathrm{C} 30-\mathrm{C} 31$ & $-151.3(3)$ \\
\hline $\mathrm{C} 42-\mathrm{C} 29-\mathrm{C} 30-\mathrm{C} 31$ & $29.7(4)$ \\
\hline
\end{tabular}

\begin{tabular}{|c|c|}
\hline $\mathrm{C} 67-\mathrm{C} 68-\mathrm{C} 69-\mathrm{C} 70$ & $1.3(6)$ \\
\hline $\mathrm{C} 66-\mathrm{C} 65-\mathrm{C} 70-\mathrm{C} 69$ & $-3.7(5)$ \\
\hline $\mathrm{C} 64-\mathrm{C} 65-\mathrm{C} 70-\mathrm{C} 69$ & $174.3(3)$ \\
\hline $\mathrm{C} 66-\mathrm{C} 65-\mathrm{C} 70-\mathrm{C} 57$ & $174.0(3)$ \\
\hline $\mathrm{C} 64-\mathrm{C} 65-\mathrm{C} 70-\mathrm{C} 57$ & $-7.9(5)$ \\
\hline $\mathrm{C} 68-\mathrm{C} 69-\mathrm{C} 70-\mathrm{C} 65$ & $1.6(5)$ \\
\hline $\mathrm{C} 68-\mathrm{C} 69-\mathrm{C} 70-\mathrm{C} 57$ & $-176.0(3)$ \\
\hline $\mathrm{N} 9-\mathrm{C} 57-\mathrm{C} 70-\mathrm{C} 65$ & $173.9(3)$ \\
\hline $\mathrm{C} 58-\mathrm{C} 57-\mathrm{C} 70-\mathrm{C} 65$ & $-9.0(5)$ \\
\hline N9-C57-C70-C69 & $-8.5(6)$ \\
\hline $\mathrm{C} 58-\mathrm{C} 57-\mathrm{C} 70-\mathrm{C} 69$ & $168.6(3)$ \\
\hline $\mathrm{C} 77-\mathrm{C} 72-\mathrm{C} 73-\mathrm{C} 74$ & $1.6(7)$ \\
\hline $\mathrm{C} 71-\mathrm{C} 72-\mathrm{C} 73-\mathrm{C} 74$ & $-178.3(5)$ \\
\hline $\mathrm{C} 72-\mathrm{C} 73-\mathrm{C} 74-\mathrm{C} 75$ & $0.3(7)$ \\
\hline $\mathrm{C} 73-\mathrm{C} 74-\mathrm{C} 75-\mathrm{C} 76$ & $-1.4(7)$ \\
\hline $\mathrm{C} 74-\mathrm{C} 75-\mathrm{C} 76-\mathrm{C} 77$ & $0.5(8)$ \\
\hline $\mathrm{C} 75-\mathrm{C} 76-\mathrm{C} 77-\mathrm{C} 72$ & $1.5(9)$ \\
\hline $\mathrm{C} 73-\mathrm{C} 72-\mathrm{C} 77-\mathrm{C} 76$ & $-2.5(8)$ \\
\hline $\mathrm{C} 71-\mathrm{C} 72-\mathrm{C} 77-\mathrm{C} 76$ & $177.3(6)$ \\
\hline $\mathrm{C} 84-\mathrm{C} 79-\mathrm{C} 80-\mathrm{C} 81$ & $0.2(4)$ \\
\hline $\mathrm{C} 78-\mathrm{C} 79-\mathrm{C} 80-\mathrm{C} 81$ & $-179.8(3)$ \\
\hline $\mathrm{C} 79-\mathrm{C} 80-\mathrm{C} 81-\mathrm{C} 82$ & $-0.6(6)$ \\
\hline $\mathrm{C} 80-\mathrm{C} 81-\mathrm{C} 82-\mathrm{C} 83$ & $0.7(7)$ \\
\hline $\mathrm{C} 81-\mathrm{C} 82-\mathrm{C} 83-\mathrm{C} 84$ & $-0.4(8)$ \\
\hline $\mathrm{C} 82-\mathrm{C} 83-\mathrm{C} 84-\mathrm{C} 79$ & $0.1(7)$ \\
\hline $\mathrm{C} 80-\mathrm{C} 79-\mathrm{C} 84-\mathrm{C} 83$ & $0.0(5)$ \\
\hline $\mathrm{C} 78-\mathrm{C} 79-\mathrm{C} 84-\mathrm{C} 83$ & $-180.0(3)$ \\
\hline $\mathrm{C} 84 \mathrm{~A}-\mathrm{C} 79 \mathrm{~A}-\mathrm{C} 80 \mathrm{~A}-\mathrm{C} 81 \mathrm{~A}$ & $0.3(6)$ \\
\hline $\mathrm{C} 78 \mathrm{~A}-\mathrm{C} 79 \mathrm{~A}-\mathrm{C} 80 \mathrm{~A}-\mathrm{C} 81 \mathrm{~A}$ & $-179.9(2)$ \\
\hline $\mathrm{C} 82 \mathrm{~A}-\mathrm{C} 81 \mathrm{~A}-\mathrm{C} 80 \mathrm{~A}-\mathrm{C} 79 \mathrm{~A}$ & $0.0(6)$ \\
\hline $\mathrm{C} 80 \mathrm{~A}-\mathrm{C} 81 \mathrm{~A}-\mathrm{C} 82 \mathrm{~A}-\mathrm{C} 83 \mathrm{~A}$ & $-0.5(7)$ \\
\hline $\mathrm{C} 81 \mathrm{~A}-\mathrm{C} 82 \mathrm{~A}-\mathrm{C} 83 \mathrm{~A}-\mathrm{C} 84 \mathrm{~A}$ & $0.5(8)$ \\
\hline $\mathrm{C} 80 \mathrm{~A}-\mathrm{C} 79 \mathrm{~A}-\mathrm{C} 84 \mathrm{~A}-\mathrm{C} 83 \mathrm{~A}$ & $-0.2(7)$ \\
\hline $\mathrm{C} 78 \mathrm{~A}-\mathrm{C} 79 \mathrm{~A}-\mathrm{C} 84 \mathrm{~A}-\mathrm{C} 83 \mathrm{~A}$ & $180.0(4)$ \\
\hline $\mathrm{C} 82 \mathrm{~A}-\mathrm{C} 83 \mathrm{~A}-\mathrm{C} 84 \mathrm{~A}-\mathrm{C} 79 \mathrm{~A}$ & $-0.2(8)$ \\
\hline $\mathrm{C} 14-\mathrm{C} 1-\mathrm{N} 1-\mathrm{O} 1$ & $-1.9(5)$ \\
\hline $\mathrm{C} 2-\mathrm{C} 1-\mathrm{N} 1-\mathrm{O} 1$ & $177.3(3)$ \\
\hline $\mathrm{C} 14-\mathrm{C} 1-\mathrm{N} 1-\mathrm{Ni} 1$ & $176.6(3)$ \\
\hline $\mathrm{C} 2-\mathrm{C} 1-\mathrm{N} 1-\mathrm{Ni} 1$ & $-4.2(4)$ \\
\hline $\mathrm{C} 3-\mathrm{C} 2-\mathrm{N} 2-\mathrm{O} 2$ & $-9.3(5)$ \\
\hline $\mathrm{C} 1-\mathrm{C} 2-\mathrm{N} 2-\mathrm{O} 2$ & $177.6(3)$ \\
\hline $\mathrm{C} 3-\mathrm{C} 2-\mathrm{N} 2-\mathrm{Ni} 1$ & $166.7(3)$ \\
\hline $\mathrm{C} 1-\mathrm{C} 2-\mathrm{N} 2-\mathrm{Ni} 1$ & $-6.4(4)$ \\
\hline $\mathrm{C} 16-\mathrm{C} 15-\mathrm{N} 3-\mathrm{O} 3$ & $173.6(3)$ \\
\hline $\mathrm{C} 28-\mathrm{C} 15-\mathrm{N} 3-\mathrm{O} 3$ & $-6.2(5)$ \\
\hline $\mathrm{C} 16-\mathrm{C} 15-\mathrm{N} 3-\mathrm{Ni} 2$ & $-13.4(4)$ \\
\hline $\mathrm{C} 28-\mathrm{C} 15-\mathrm{N} 3-\mathrm{Ni} 2$ & $166.8(2)$ \\
\hline $\mathrm{C} 15-\mathrm{C} 16-\mathrm{N} 4-\mathrm{O} 4$ & $-4.3(5)$ \\
\hline
\end{tabular}




\begin{tabular}{|c|c|}
\hline $\mathrm{N} 6-\mathrm{C} 30-\mathrm{C} 31-\mathrm{C} 32$ & $-24.0(5)$ \\
\hline $\mathrm{C} 29-\mathrm{C} 30-\mathrm{C} 31-\mathrm{C} 32$ & $155.8(3)$ \\
\hline $\mathrm{N} 6-\mathrm{C} 30-\mathrm{C} 31-\mathrm{C} 36$ & $155.9(3)$ \\
\hline $\mathrm{C} 29-\mathrm{C} 30-\mathrm{C} 31-\mathrm{C} 36$ & $-24.4(5)$ \\
\hline $\mathrm{C} 36-\mathrm{C} 31-\mathrm{C} 32-\mathrm{C} 33$ & $-1.1(6)$ \\
\hline $\mathrm{C} 30-\mathrm{C} 31-\mathrm{C} 32-\mathrm{C} 33$ & $178.8(4)$ \\
\hline $\mathrm{C} 31-\mathrm{C} 32-\mathrm{C} 33-\mathrm{C} 34$ & $-0.1(7)$ \\
\hline $\mathrm{C} 32-\mathrm{C} 33-\mathrm{C} 34-\mathrm{C} 35$ & $0.8(7)$ \\
\hline $\mathrm{C} 33-\mathrm{C} 34-\mathrm{C} 35-\mathrm{C} 36$ & $-0.3(7)$ \\
\hline $\mathrm{C} 32-\mathrm{C} 31-\mathrm{C} 36-\mathrm{C} 35$ & $1.5(5)$ \\
\hline $\mathrm{C} 30-\mathrm{C} 31-\mathrm{C} 36-\mathrm{C} 35$ & $-178.3(3)$ \\
\hline $\mathrm{C} 32-\mathrm{C} 31-\mathrm{C} 36-\mathrm{C} 37$ & $-177.9(3)$ \\
\hline $\mathrm{C} 30-\mathrm{C} 31-\mathrm{C} 36-\mathrm{C} 37$ & $2.3(5)$ \\
\hline $\mathrm{C} 34-\mathrm{C} 35-\mathrm{C} 36-\mathrm{C} 31$ & $-0.8(6)$ \\
\hline $\mathrm{C} 34-\mathrm{C} 35-\mathrm{C} 36-\mathrm{C} 37$ & $178.5(4)$ \\
\hline $\mathrm{C} 31-\mathrm{C} 36-\mathrm{C} 37-\mathrm{C} 38$ & $-166.6(3)$ \\
\hline $\mathrm{C} 35-\mathrm{C} 36-\mathrm{C} 37-\mathrm{C} 38$ & $14.1(5)$ \\
\hline $\mathrm{C} 31-\mathrm{C} 36-\mathrm{C} 37-\mathrm{C} 42$ & $14.3(5)$ \\
\hline $\mathrm{C} 35-\mathrm{C} 36-\mathrm{C} 37-\mathrm{C} 42$ & $-165.1(3)$ \\
\hline $\mathrm{C} 42-\mathrm{C} 37-\mathrm{C} 38-\mathrm{C} 39$ & $1.1(5)$ \\
\hline $\mathrm{C} 36-\mathrm{C} 37-\mathrm{C} 38-\mathrm{C} 39$ & $-178.0(3)$ \\
\hline $\mathrm{C} 37-\mathrm{C} 38-\mathrm{C} 39-\mathrm{C} 40$ & $0.4(6)$ \\
\hline $\mathrm{C} 38-\mathrm{C} 39-\mathrm{C} 40-\mathrm{C} 41$ & $-1.6(6)$ \\
\hline $\mathrm{C} 39-\mathrm{C} 40-\mathrm{C} 41-\mathrm{C} 42$ & $1.2(6)$ \\
\hline $\mathrm{C} 40-\mathrm{C} 41-\mathrm{C} 42-\mathrm{C} 37$ & $0.3(5)$ \\
\hline $\mathrm{C} 40-\mathrm{C} 41-\mathrm{C} 42-\mathrm{C} 29$ & $-173.5(3)$ \\
\hline $\mathrm{C} 38-\mathrm{C} 37-\mathrm{C} 42-\mathrm{C} 41$ & $-1.5(5)$ \\
\hline $\mathrm{C} 36-\mathrm{C} 37-\mathrm{C} 42-\mathrm{C} 41$ & $177.7(3)$ \\
\hline $\mathrm{C} 38-\mathrm{C} 37-\mathrm{C} 42-\mathrm{C} 29$ & $172.6(3)$ \\
\hline $\mathrm{C} 36-\mathrm{C} 37-\mathrm{C} 42-\mathrm{C} 29$ & $-8.3(5)$ \\
\hline $\mathrm{N} 5-\mathrm{C} 29-\mathrm{C} 42-\mathrm{C} 41$ & $-18.5(5)$ \\
\hline $\mathrm{C} 30-\mathrm{C} 29-\mathrm{C} 42-\mathrm{C} 41$ & $160.4(3)$ \\
\hline N5-C29-C42-C37 & $167.6(3)$ \\
\hline $\mathrm{C} 30-\mathrm{C} 29-\mathrm{C} 42-\mathrm{C} 37$ & $-13.4(4)$ \\
\hline $\mathrm{N} 7-\mathrm{C} 43-\mathrm{C} 44-\mathrm{N} 8$ & $8.4(4)$ \\
\hline $\mathrm{C} 56-\mathrm{C} 43-\mathrm{C} 44-\mathrm{N} 8$ & $-169.7(3)$ \\
\hline N7-C43-C44-C45 & $-167.8(3)$ \\
\hline $\mathrm{C} 56-\mathrm{C} 43-\mathrm{C} 44-\mathrm{C} 45$ & $14.1(5)$ \\
\hline $\mathrm{N} 8-\mathrm{C} 44-\mathrm{C} 45-\mathrm{C} 46$ & $-6.1(6)$ \\
\hline $\mathrm{C} 43-\mathrm{C} 44-\mathrm{C} 45-\mathrm{C} 46$ & $169.4(3)$ \\
\hline $\mathrm{N} 8-\mathrm{C} 44-\mathrm{C} 45-\mathrm{C} 50$ & $178.3(3)$ \\
\hline $\mathrm{C} 43-\mathrm{C} 44-\mathrm{C} 45-\mathrm{C} 50$ & $-6.2(5)$ \\
\hline $\mathrm{C} 50-\mathrm{C} 45-\mathrm{C} 46-\mathrm{C} 47$ & $1.8(6)$ \\
\hline $\mathrm{C} 44-\mathrm{C} 45-\mathrm{C} 46-\mathrm{C} 47$ & $-173.7(4)$ \\
\hline $\mathrm{C} 45-\mathrm{C} 46-\mathrm{C} 47-\mathrm{C} 48$ & $-1.8(6)$ \\
\hline $\mathrm{C} 46-\mathrm{C} 47-\mathrm{C} 48-\mathrm{C} 49$ & $1.1(7)$ \\
\hline $\mathrm{C} 47-\mathrm{C} 48-\mathrm{C} 49-\mathrm{C} 50$ & $-0.4(7)$ \\
\hline & \\
\hline
\end{tabular}

\begin{tabular}{|c|c|}
\hline $\mathrm{C} 17-\mathrm{C} 16-\mathrm{N} 4-\mathrm{O} 4$ & $175.4(3)$ \\
\hline $\mathrm{C} 30-\mathrm{C} 29-\mathrm{N} 5-\mathrm{O} 5$ & $172.0(3)$ \\
\hline $\mathrm{C} 42-\mathrm{C} 29-\mathrm{N} 5-\mathrm{O} 5$ & $-9.0(5)$ \\
\hline $\mathrm{C} 30-\mathrm{C} 29-\mathrm{N} 5-\mathrm{Ni} 1$ & $-18.5(4)$ \\
\hline $\mathrm{C} 42-\mathrm{C} 29-\mathrm{N} 5-\mathrm{Ni} 1$ & $160.5(3)$ \\
\hline $\mathrm{C} 29-\mathrm{C} 30-\mathrm{N} 6-\mathrm{O} 6$ & $-4.0(5)$ \\
\hline $\mathrm{C} 31-\mathrm{C} 30-\mathrm{N} 6-\mathrm{O} 6$ & $175.7(3)$ \\
\hline $\mathrm{C} 44-\mathrm{C} 43-\mathrm{N} 7-\mathrm{O} 7$ & $178.7(3)$ \\
\hline $\mathrm{C} 56-\mathrm{C} 43-\mathrm{N} 7-\mathrm{O} 7$ & $-3.4(5)$ \\
\hline $\mathrm{C} 44-\mathrm{C} 43-\mathrm{N} 7-\mathrm{Ni} 2$ & $-6.0(4)$ \\
\hline $\mathrm{C} 56-\mathrm{C} 43-\mathrm{N} 7-\mathrm{Ni} 2$ & $172.0(3)$ \\
\hline $\mathrm{C} 43-\mathrm{C} 44-\mathrm{N} 8-\mathrm{O} 8$ & $175.6(3)$ \\
\hline $\mathrm{C} 45-\mathrm{C} 44-\mathrm{N} 8-\mathrm{O} 8$ & $-8.5(5)$ \\
\hline $\mathrm{C} 43-\mathrm{C} 44-\mathrm{N} 8-\mathrm{Ni} 2$ & $-7.2(4)$ \\
\hline $\mathrm{C} 45-\mathrm{C} 44-\mathrm{N} 8-\mathrm{Ni} 2$ & $168.7(3)$ \\
\hline $\mathrm{C} 70-\mathrm{C} 57-\mathrm{N} 9-\mathrm{O} 9$ & $-0.4(5)$ \\
\hline $\mathrm{C} 58-\mathrm{C} 57-\mathrm{N} 9-\mathrm{O} 9$ & $-177.7(3)$ \\
\hline $\mathrm{C} 70-\mathrm{C} 57-\mathrm{N} 9-\mathrm{Ni} 3$ & $166.5(3)$ \\
\hline $\mathrm{C} 58-\mathrm{C} 57-\mathrm{N} 9-\mathrm{Ni} 3$ & $-10.8(4)$ \\
\hline $\mathrm{C} 59-\mathrm{C} 58-\mathrm{N} 10-\mathrm{O} 10$ & $-3.5(5)$ \\
\hline $\mathrm{C} 57-\mathrm{C} 58-\mathrm{N} 10-\mathrm{O} 10$ & $178.9(3)$ \\
\hline $\mathrm{C} 59-\mathrm{C} 58-\mathrm{N} 10-\mathrm{Ni3}$ & $162.1(3)$ \\
\hline $\mathrm{C} 57-\mathrm{C} 58-\mathrm{N} 10-\mathrm{Ni3}$ & $-15.6(4)$ \\
\hline $\mathrm{C} 2-\mathrm{N} 2-\mathrm{O} 2-\mathrm{Ni} 3$ & $143.0(3)$ \\
\hline $\mathrm{Ni} 1-\mathrm{N} 2-\mathrm{O} 2-\mathrm{Ni3}$ & $-32.7(3)$ \\
\hline $\mathrm{C} 16-\mathrm{N} 4-\mathrm{O} 4-\mathrm{Ni} 2$ & $-30.5(4)$ \\
\hline $\mathrm{C} 16-\mathrm{N} 4-\mathrm{O} 4-\mathrm{Ni} 3$ & $148.7(2)$ \\
\hline $\mathrm{C} 30-\mathrm{N} 6-\mathrm{O} 6-\mathrm{Ni1}$ & $-30.9(4)$ \\
\hline $\mathrm{C} 30-\mathrm{N} 6-\mathrm{O} 6-\mathrm{Ni} 3$ & $145.4(2)$ \\
\hline $\mathrm{C} 44-\mathrm{N} 8-\mathrm{O} 8-\mathrm{Ni} 3$ & $145.0(3)$ \\
\hline $\mathrm{Ni} 2-\mathrm{N} 8-\mathrm{O} 8-\mathrm{Ni} 3$ & $-32.1(3)$ \\
\hline $\mathrm{N} 6-\mathrm{O} 6-\mathrm{Ni} 1-\mathrm{N} 1$ & $-158.8(6)$ \\
\hline $\mathrm{Ni3}-\mathrm{O} 6-\mathrm{Ni} 1-\mathrm{N} 1$ & $24.9(7)$ \\
\hline $\mathrm{N} 6-\mathrm{O} 6-\mathrm{Ni1}-\mathrm{N} 2$ & $-151.0(3)$ \\
\hline $\mathrm{Ni3}-\mathrm{O} 6-\mathrm{Ni} 1-\mathrm{N} 2$ & $32.64(14)$ \\
\hline $\mathrm{N} 6-\mathrm{O} 6-\mathrm{Ni} 1-\mathrm{N} 5$ & $31.9(3)$ \\
\hline $\mathrm{Ni3}-\mathrm{O} 6-\mathrm{Ni1}-\mathrm{N} 5$ & $-144.41(14)$ \\
\hline $\mathrm{C} 1-\mathrm{N} 1-\mathrm{Ni} 1-\mathrm{O} 6$ & $8.5(8)$ \\
\hline $\mathrm{O} 1-\mathrm{N} 1-\mathrm{Ni1}-\mathrm{O} 6$ & $-173.0(6)$ \\
\hline $\mathrm{C} 1-\mathrm{N} 1-\mathrm{N} 11-\mathrm{N} 2$ & $0.7(3)$ \\
\hline $\mathrm{O} 1-\mathrm{N} 1-\mathrm{N} 11-\mathrm{N} 2$ & $179.1(3)$ \\
\hline $\mathrm{C} 1-\mathrm{N} 1-\mathrm{Ni} 1-\mathrm{N} 5$ & $177.7(3)$ \\
\hline $\mathrm{O} 1-\mathrm{N} 1-\mathrm{Ni} 1-\mathrm{N} 5$ & $-3.9(3)$ \\
\hline $\mathrm{C} 2-\mathrm{N} 2-\mathrm{Ni} 1-\mathrm{O} 6$ & $-175.1(2)$ \\
\hline $\mathrm{O} 2-\mathrm{N} 2-\mathrm{Ni1}-\mathrm{O} 6$ & $0.8(2)$ \\
\hline $\mathrm{C} 2-\mathrm{N} 2-\mathrm{Ni} 1-\mathrm{N} 1$ & $3.5(2)$ \\
\hline $\mathrm{O} 2-\mathrm{N} 2-\mathrm{Ni} 1-\mathrm{N} 1$ & $179.4(3)$ \\
\hline $\mathrm{C} 29-\mathrm{N} 5-\mathrm{Ni} 1-\mathrm{O} 6$ & $-5.9(3)$ \\
\hline
\end{tabular}




\begin{tabular}{|c|c|}
\hline $\mathrm{C} 48-\mathrm{C} 49-\mathrm{C} 50-\mathrm{C} 51$ & $179.7(4)$ \\
\hline $\mathrm{C} 46-\mathrm{C} 45-\mathrm{C} 50-\mathrm{C} 49$ & $-1.0(5)$ \\
\hline $\mathrm{C} 44-\mathrm{C} 45-\mathrm{C} 50-\mathrm{C} 49$ & $174.6(3)$ \\
\hline $\mathrm{C} 46-\mathrm{C} 45-\mathrm{C} 50-\mathrm{C} 51$ & $179.7(3)$ \\
\hline $\mathrm{C} 44-\mathrm{C} 45-\mathrm{C} 50-\mathrm{C} 51$ & $-4.7(5)$ \\
\hline $\mathrm{C} 49-\mathrm{C} 50-\mathrm{C} 51-\mathrm{C} 56$ & $-171.2(4)$ \\
\hline $\mathrm{C} 45-\mathrm{C} 50-\mathrm{C} 51-\mathrm{C} 56$ & $8.0(5)$ \\
\hline $\mathrm{C} 49-\mathrm{C} 50-\mathrm{C} 51-\mathrm{C} 52$ & $9.9(6)$ \\
\hline $\mathrm{C} 45-\mathrm{C} 50-\mathrm{C} 51-\mathrm{C} 52$ & $-170.8(3)$ \\
\hline $\mathrm{C} 56-\mathrm{C} 51-\mathrm{C} 52-\mathrm{C} 53$ & $-0.1(6)$ \\
\hline $\mathrm{C} 50-\mathrm{C} 51-\mathrm{C} 52-\mathrm{C} 53$ & $178.7(4)$ \\
\hline $\mathrm{C} 51-\mathrm{C} 52-\mathrm{C} 53-\mathrm{C} 54$ & $0.2(6)$ \\
\hline $\mathrm{C} 52-\mathrm{C} 53-\mathrm{C} 54-\mathrm{C} 55$ & $-0.1(6)$ \\
\hline $\mathrm{C} 53-\mathrm{C} 54-\mathrm{C} 55-\mathrm{C} 56$ & $-0.1(6)$ \\
\hline $\mathrm{C} 54-\mathrm{C} 55-\mathrm{C} 56-\mathrm{C} 51$ & $0.2(5)$ \\
\hline $\mathrm{C} 54-\mathrm{C} 55-\mathrm{C} 56-\mathrm{C} 43$ & $-178.5(3)$ \\
\hline $\mathrm{C} 52-\mathrm{C} 51-\mathrm{C} 56-\mathrm{C} 55$ & $-0.1(5)$ \\
\hline $\mathrm{C} 50-\mathrm{C} 51-\mathrm{C} 56-\mathrm{C} 55$ & $-179.0(3)$ \\
\hline $\mathrm{C} 52-\mathrm{C} 51-\mathrm{C} 56-\mathrm{C} 43$ & $178.7(3)$ \\
\hline
\end{tabular}

$\begin{array}{ll}\mathrm{O} 5-\mathrm{N} 5-\mathrm{Ni} 1-\mathrm{O} 6 & 163.6(3) \\ \mathrm{C} 29-\mathrm{N} 5-\mathrm{Ni} 1-\mathrm{N} 1 & 176.0(3) \\ \mathrm{O} 5-\mathrm{N} 5-\mathrm{Ni} 1-\mathrm{N} 1 & -14.5(3) \\ \mathrm{N} 4-\mathrm{O} 4-\mathrm{Ni} 2-\mathrm{N} 8 & -149.4(3) \\ \mathrm{Ni} 3-\mathrm{O} 4-\mathrm{Ni2}-\mathrm{N} 8 & 31.36(14) \\ \mathrm{N} 4-\mathrm{O} 4-\mathrm{Ni} 2-\mathrm{N} 3 & 33.3(3) \\ \mathrm{Ni3}-\mathrm{O} 4-\mathrm{Ni2}-\mathrm{N} 3 & -145.90(14) \\ \mathrm{C} 43-\mathrm{N} 7-\mathrm{Ni2}-\mathrm{N} 8 & 1.8(3) \\ \mathrm{O} 7-\mathrm{N} 7-\mathrm{Ni2}-\mathrm{N} 8 & 177.1(3) \\ \mathrm{C} 43-\mathrm{N} 7-\mathrm{Ni2}-\mathrm{N} 3 & 179.0(3) \\ \mathrm{O} 7-\mathrm{N} 7-\mathrm{Ni2}-\mathrm{N} 3 & -5.7(3) \\ \mathrm{C} 44-\mathrm{N} 8-\mathrm{Ni2}-\mathrm{O} 4 & -176.1(3) \\ \mathrm{O} 8-\mathrm{N} 8-\mathrm{Ni2}-\mathrm{O} 4 & 1.1(2) \\ \mathrm{C} 44-\mathrm{N} 8-\mathrm{Ni2}-\mathrm{N} 7 & 3.3(2) \\ \text { O8-N8-Ni2-N7 } & -179.5(3) \\ \mathrm{C} 15-\mathrm{N} 3-\mathrm{Ni2}-\mathrm{O} 4 & -10.2(3) \\ \text { O3-N3-Ni2-O4 } & 162.8(2) \\ \text { C15-N3-Ni2-N7 } & 170.8(3) \\ \text { O3-N3-Ni2-N7 } & -16.1(2)\end{array}$

Hydrogen-bond geometry $\left(\AA,{ }^{o}\right)$

\begin{tabular}{lllll}
\hline$D-\mathrm{H} \cdots A$ & $D-\mathrm{H}$ & $\mathrm{H} \cdots A$ & $D \cdots A$ & $D-\mathrm{H} \cdots A$ \\
\hline $\mathrm{O} 1-\mathrm{H} 1 A \cdots \mathrm{O} 5$ & $1.29(6)$ & $1.13(6)$ & $2.405(4)$ & $169(6)$ \\
$\mathrm{O} 3-\mathrm{H} 2 A \cdots \mathrm{O} 7$ & $1.17(5)$ & $1.23(5)$ & $2.402(4)$ & $176(5)$ \\
$\mathrm{O}-\mathrm{H} 3 A \cdots \mathrm{N} 4$ & $0.86(6)$ & $1.82(6)$ & $2.672(4)$ & $175(7)$ \\
$\mathrm{O} 10-\mathrm{H} 4 A \cdots \mathrm{N} 6$ & $1.04(5)$ & $1.63(5)$ & $2.657(4)$ & $168(4)$ \\
\hline
\end{tabular}

(2) $\operatorname{Bis}\left(\mu_{2}\right.$-bis $\{[10$-(oxidoimino)-9,10-dihydrophenanthren-9-ylidene]amino\}difluoroborato)(phenanthrene-9,10dione dioxime)trinickel(II) dichloromethane trisolvate

Crystal data

$\left[\mathrm{Ni}_{3}\left(\mathrm{C}_{28} \mathrm{H}_{16} \mathrm{BF}_{2} \mathrm{~N}_{4} \mathrm{O}_{4}\right)_{2}\left(\mathrm{C}_{14} \mathrm{H}_{10} \mathrm{~N}_{2} \mathrm{O}_{2}\right)\right] \cdot 3 \mathrm{CH}_{2} \mathrm{Cl}_{2}$

$F(000)=2968$

$M_{r}=1456.88$

Monoclinic, $P 2{ }_{1} / c$

$a=15.6414(8) \AA$

$b=30.8358(11) \AA$

$c=14.7380(8) \AA$

$\beta=112.411(6)^{\circ}$

$V=6571.5(6) \AA^{3}$

$D_{\mathrm{x}}=1.473 \mathrm{Mg} \mathrm{m}^{-3}$

$\mathrm{Cu} K \alpha$ radiation, $\lambda=1.54184 \AA$

Cell parameters from 25728 reflections

$\theta=3.4-76.5^{\circ}$

$\mu=1.67 \mathrm{~mm}^{-1}$

$T=100 \mathrm{~K}$

$Z=4$

Block, red

$0.29 \times 0.07 \times 0.04 \mathrm{~mm}$

\section{Data collection}

Agilent SuperNova Dual Source diffractometer with an Atlas detector

Radiation source: sealed X-ray tube Detector resolution: 5.2940 pixels $\mathrm{mm}^{-1}$ $\omega$ scans

\footnotetext{
Absorption correction: multi-scan

(CrysAlis PRO; Agilent, 2014)

$T_{\min }=0.303, T_{\max }=1.000$

25337 measured reflections

13041 independent reflections

8782 reflections with $I>2 \sigma(I)$
} 


\author{
$R_{\text {int }}=0.060$ \\ $\theta_{\max }=75.0^{\circ}, \theta_{\min }=3.4^{\circ}$ \\ $h=-19 \rightarrow 19$

\section{Refinement} \\ Refinement on $F^{2}$ \\ Least-squares matrix: full \\ $R\left[F^{2}>2 \sigma\left(F^{2}\right)\right]=0.077$ \\ $w R\left(F^{2}\right)=0.226$ \\ $S=1.03$ \\ 13041 reflections \\ 896 parameters \\ 0 restraints
}

$k=-26 \rightarrow 38$
$l=-18 \rightarrow 18$

Hydrogen site location: inferred from neighbouring sites

$\mathrm{H}$ atoms treated by a mixture of independent and constrained refinement

$w=1 /\left[\sigma^{2}\left(F_{\mathrm{o}}{ }^{2}\right)+(0.108 P)^{2}+8.2009 P\right]$ where $P=\left(F_{\mathrm{o}}^{2}+2 F_{\mathrm{c}}^{2}\right) / 3$

$(\Delta / \sigma)_{\max }=0.006$

$\Delta \rho_{\max }=1.16 \mathrm{e} \AA^{-3}$

$\Delta \rho_{\min }=-0.83$ e $\AA^{-3}$

Special details

Experimental. CrysAlisPro. (Agilent, 2014). Empirical absorption correction using spherical harmonics, implemented in SCALE3 ABSPACK scaling algorithm.

Geometry. All esds (except the esd in the dihedral angle between two 1.s. planes) are estimated using the full covariance matrix. The cell esds are taken into account individually in the estimation of esds in distances, angles and torsion angles; correlations between esds in cell parameters are only used when they are defined by crystal symmetry. An approximate (isotropic) treatment of cell esds is used for estimating esds involving l.s. planes.

Refinement. Refinement of $\mathrm{F}^{2}$ against ALL reflections. The weighted R-factor $\mathrm{wR}$ and goodness of fit $\mathrm{S}$ are based on $\mathrm{F}^{2}$, conventional R-factors $R$ are based on $F$, with $F$ set to zero for negative $F^{2}$. The threshold expression of $F^{2}>2 \operatorname{sigma}\left(F^{2}\right)$ is used only for calculating R-factors(gt) etc. and is not relevant to the choice of reflections for refinement. R-factors based on $\mathrm{F}^{2}$ are statistically about twice as large as those based on F, and R- factors based on ALL data will be even larger. Three molecules of what appeared to be dichloromethane were found to be badly disordered. Attempts to model the disorder were unsatisfactory. The contributions to the scattering factors due to these solvent molecules were removed by use of the utility SQUEEZE (Sluis and Spek, 1990) in PLATON98 (Spek, 1998). PLATON98 was used as incorporated in WinGX (Farrugia, 1999).

Fractional atomic coordinates and isotropic or equivalent isotropic displacement parameters $\left(\AA^{2}\right)$

\begin{tabular}{lllll}
\hline & $x$ & $y$ & $z$ & $U_{\text {iso }}{ }^{*} / U_{\text {eq }}$ \\
\hline C1 & $0.3411(3)$ & $0.33690(15)$ & $0.7321(4)$ & $0.0323(10)$ \\
C2 & $0.4326(3)$ & $0.33998(16)$ & $0.7254(4)$ & $0.0336(11)$ \\
C3 & $0.4765(4)$ & $0.29952(16)$ & $0.7127(4)$ & $0.0347(11)$ \\
C4 & $0.5720(4)$ & $0.29722(18)$ & $0.7393(4)$ & $0.0423(12)$ \\
H4 & 0.6093 & 0.3220 & 0.7661 & $0.051^{*}$ \\
C5 & $0.6130(4)$ & $0.25876(18)$ & $0.7267(5)$ & $0.0499(14)$ \\
H5 & 0.6781 & 0.2571 & 0.7446 & $0.060^{*}$ \\
C6 & $0.5574(4)$ & $0.22266(18)$ & $0.6875(5)$ & $0.0498(15)$ \\
H6 & 0.5847 & 0.1963 & 0.6785 & $0.060^{*}$ \\
C7 & $0.4637(4)$ & $0.22483(17)$ & $0.6619(4)$ & $0.0460(14)$ \\
H7 & 0.4274 & 0.1997 & 0.6361 & $0.055^{*}$ \\
C8 & $0.4198(4)$ & $0.26291(15)$ & $0.6727(4)$ & $0.0362(11)$ \\
C9 & $0.3201(4)$ & $0.26641(15)$ & $0.6448(4)$ & $0.0357(11)$ \\
C10 & $0.2594(4)$ & $0.23476(16)$ & $0.5841(4)$ & $0.0394(12)$ \\
H10 & 0.2844 & 0.2106 & 0.5627 & $0.047^{*}$ \\
C11 & $0.1655(4)$ & $0.23823(18)$ & $0.5555(4)$ & $0.0438(13)$ \\
H11 & 0.1267 & 0.2162 & 0.5158 & $0.053^{*}$
\end{tabular}




\begin{tabular}{|c|c|c|c|c|}
\hline $\mathrm{C} 12$ & $0.1264(4)$ & $0.27305(18)$ & $0.5831(4)$ & $0.0422(12)$ \\
\hline H12 & 0.0611 & 0.2754 & 0.5620 & $0.051^{*}$ \\
\hline $\mathrm{C} 13$ & $0.1837(4)$ & $0.30485(16)$ & $0.6426(4)$ & $0.0361(11)$ \\
\hline H13 & 0.1571 & 0.3291 & 0.6619 & $0.043 *$ \\
\hline $\mathrm{C} 14$ & $0.2797(4)$ & $0.30177(15)$ & $0.6744(4)$ & $0.0336(11)$ \\
\hline $\mathrm{C} 15$ & $0.2126(3)$ & $0.36498(16)$ & $0.4672(3)$ & $0.0310(10)$ \\
\hline $\mathrm{C} 16$ & 0.3085 & $0.37760(15)$ & $0.4870(3)$ & $0.0295(10)$ \\
\hline C17 & $0.3700(3)$ & $0.34790(16)$ & $0.4634(3)$ & $0.0304(10)$ \\
\hline $\mathrm{C} 18$ & $0.4630(3)$ & $0.35767(17)$ & $0.4827(4)$ & $0.0352(11)$ \\
\hline H18 & 0.4869 & 0.3853 & 0.5083 & $0.042 *$ \\
\hline C19 & $0.5208(4)$ & $0.32743(18)$ & 0.4649 (4) & $0.0442(13)$ \\
\hline H19 & 0.5832 & 0.3346 & 0.4770 & $0.053 *$ \\
\hline $\mathrm{C} 20$ & 0.4864 (4) & 0.28685 (19) & $0.4294(4)$ & $0.0472(14)$ \\
\hline $\mathrm{H} 20$ & 0.5261 & 0.2657 & 0.4196 & $0.057^{*}$ \\
\hline $\mathrm{C} 21$ & $0.3950(4)$ & $0.27698(18)$ & $0.4081(4)$ & $0.0423(12)$ \\
\hline $\mathrm{H} 21$ & 0.3725 & 0.2491 & 0.3827 & $0.051 *$ \\
\hline $\mathrm{C} 22$ & 0.3341 & $0.30700(16)$ & $0.4230(4)$ & $0.0330(10)$ \\
\hline $\mathrm{C} 23$ & $0.2352(4)$ & $0.29719(16)$ & $0.3911(4)$ & $0.0335(10)$ \\
\hline $\mathrm{C} 24$ & $0.1973(4)$ & $0.25910(18)$ & $0.3381(4)$ & $0.0423(12)$ \\
\hline $\mathrm{H} 24$ & 0.2378 & 0.2386 & 0.3275 & $0.051 *$ \\
\hline $\mathrm{C} 25$ & $0.1044(4)$ & $0.25044(19)$ & $0.3013(4)$ & $0.0464(13)$ \\
\hline $\mathrm{H} 25$ & 0.0816 & 0.2242 & 0.2667 & $0.056^{*}$ \\
\hline $\mathrm{C} 26$ & $0.0436(4)$ & 0.28018 (19) & $0.3149(4)$ & $0.0455(13)$ \\
\hline $\mathrm{H} 26$ & -0.0211 & 0.2750 & 0.2874 & $0.055^{*}$ \\
\hline $\mathrm{C} 27$ & 0.0781 & $0.31762(18)$ & $0.3690(4)$ & $0.0380(11)$ \\
\hline $\mathrm{H} 27$ & 0.0365 & 0.3376 & 0.3794 & $0.046^{*}$ \\
\hline $\mathrm{C} 28$ & $0.1730(3)$ & $0.32636(16)$ & $0.4084(4)$ & $0.0341(11)$ \\
\hline $\mathrm{C} 29$ & $0.3296(3)$ & $0.47670(15)$ & $0.9399(4)$ & $0.0325(10)$ \\
\hline $\mathrm{C} 30$ & 0.3915 & $0.50074(15)$ & $0.9040(3)$ & $0.0316(10)$ \\
\hline $\mathrm{C} 31$ & $0.4137(3)$ & $0.54622(15)$ & $0.9300(3)$ & $0.0322(10)$ \\
\hline C32 & $0.4728(4)$ & $0.57048(16)$ & $0.8987(4)$ & $0.0386(12)$ \\
\hline H32 & 0.4988 & 0.5573 & 0.8569 & $0.046^{*}$ \\
\hline $\mathrm{C} 33$ & $0.4942(4)$ & $0.61279(17)$ & $0.9267(4)$ & $0.0448(13)$ \\
\hline H33 & 0.5354 & 0.6284 & 0.9053 & $0.054 *$ \\
\hline C34 & $0.4558(5)$ & $0.63252(18)$ & $0.9862(5)$ & $0.0557(17)$ \\
\hline H34 & 0.4704 & 0.6618 & 1.0061 & $0.067 *$ \\
\hline $\mathrm{C} 35$ & $0.3969(4)$ & $0.60974(17)$ & $1.0163(4)$ & $0.0454(14)$ \\
\hline H35 & 0.3712 & 0.6239 & 1.0573 & $0.055^{*}$ \\
\hline C36 & $0.3721(4)$ & $0.56675(16)$ & $0.9903(3)$ & $0.0340(11)$ \\
\hline C37 & $0.3088(4)$ & $0.54261(16)$ & $1.0242(4)$ & $0.0360(11)$ \\
\hline $\mathrm{C} 38$ & $0.2666(4)$ & $0.56390(18)$ & $1.0798(4)$ & $0.0418(13)$ \\
\hline H38 & 0.2772 & 0.5941 & 1.0922 & $0.050 *$ \\
\hline C39 & $0.2105(4)$ & $0.5425(2)$ & $1.1168(4)$ & $0.0497(15)$ \\
\hline H39 & 0.1828 & 0.5579 & 1.1545 & $0.060 *$ \\
\hline $\mathrm{C} 40$ & 0.1938 & $0.4987(2)$ & 1.0998 (4) & $0.0443(13)$ \\
\hline $\mathrm{H} 40$ & 0.1552 & 0.4838 & 1.1261 & $0.053^{*}$ \\
\hline $\mathrm{C} 41$ & $0.2339(4)$ & $0.47662(17)$ & $1.0439(4)$ & $0.0372(11)$ \\
\hline H41 & 0.2230 & 0.4464 & 1.0326 & $0.045^{*}$ \\
\hline
\end{tabular}




\begin{tabular}{|c|c|c|c|c|}
\hline $\mathrm{C} 42$ & $0.2897(4)$ & $0.49811(16)$ & $1.0043(3)$ & $0.0355(11)$ \\
\hline $\mathrm{C} 43$ & $0.1661(3)$ & $0.49600(19)$ & 0.6807 (4) & $0.0400(12)$ \\
\hline $\mathrm{C} 44$ & $0.2317(3)$ & $0.52960(18)$ & $0.6755(4)$ & $0.0392(12)$ \\
\hline $\mathrm{C} 45$ & $0.2153(4)$ & $0.5756(2)$ & $0.6941(4)$ & $0.0511(16)$ \\
\hline $\mathrm{C} 46$ & $0.2581(4)$ & $0.6091(2)$ & $0.6633(5)$ & $0.0581(18)$ \\
\hline H46 & 0.2979 & 0.6023 & 0.6301 & $0.070^{*}$ \\
\hline $\mathrm{C} 47$ & $0.2434(5)$ & $0.6522(2)$ & $0.6804(7)$ & $0.077(3)$ \\
\hline H47 & 0.2730 & 0.6749 & 0.6597 & $0.093^{*}$ \\
\hline $\mathrm{C} 48$ & $0.1844(5)$ & $0.6615(3)$ & $0.7286(7)$ & $0.094(3)$ \\
\hline H48 & 0.1729 & 0.6908 & 0.7403 & $0.112 *$ \\
\hline C49 & $0.1427(5)$ & $0.6285(3)$ & $0.7595(6)$ & $0.083(3)$ \\
\hline H49 & 0.1032 & 0.6356 & 0.7928 & $0.100^{*}$ \\
\hline $\mathrm{C} 50$ & $0.1571(4)$ & $0.5845(2)$ & $0.7429(5)$ & $0.061(2)$ \\
\hline C51 & $0.1151(4)$ & $0.5488(3)$ & $0.7768(4)$ & $0.062(2)$ \\
\hline C52 & $0.0734(5)$ & $0.5578(3)$ & $0.8439(5)$ & $0.073(2)$ \\
\hline H52 & 0.0721 & 0.5866 & 0.8658 & $0.088^{*}$ \\
\hline $\mathrm{C} 53$ & $0.0348(5)$ & $0.5250(4)$ & $0.8776(5)$ & $0.088(3)$ \\
\hline H53 & 0.0047 & 0.5317 & 0.9209 & $0.105^{*}$ \\
\hline $\mathrm{C} 54$ & $0.0385(4)$ & $0.4820(4)$ & $0.8502(5)$ & $0.077(3)$ \\
\hline H54 & 0.0127 & 0.4596 & 0.8761 & $0.093^{*}$ \\
\hline C55 & $0.0804(4)$ & $0.4722(3)$ & $0.7841(4)$ & $0.0604(19)$ \\
\hline H55 & 0.0838 & 0.4430 & 0.7650 & $0.072^{*}$ \\
\hline C56 & $0.1175(4)$ & $0.5059(2)$ & $0.7462(4)$ & $0.0500(16)$ \\
\hline $\mathrm{C} 57$ & $0.6326(3)$ & $0.48466(16)$ & $0.6652(4)$ & $0.0315(10)$ \\
\hline C58 & $0.5935(3)$ & $0.52870(16)$ & $0.6698(3)$ & $0.0315(10)$ \\
\hline C59 & $0.6591(3)$ & $0.56487(15)$ & $0.7051(3)$ & $0.0304(10)$ \\
\hline C60 & $0.6390(4)$ & $0.60134(17)$ & 0.7479 (4) & $0.0395(12)$ \\
\hline H60 & 0.5788 & 0.6050 & 0.7481 & $0.047^{*}$ \\
\hline C61 & $0.7056(4)$ & $0.63235(18)$ & $0.7902(5)$ & $0.0463(13)$ \\
\hline H61 & 0.6915 & 0.6570 & 0.8207 & $0.056^{*}$ \\
\hline C62 & $0.7926(4)$ & $0.62788(17)$ & $0.7885(4)$ & $0.0436(13)$ \\
\hline H62 & 0.8380 & 0.6496 & 0.8171 & $0.052 *$ \\
\hline C63 & $0.8141(4)$ & $0.59185(17)$ & $0.7452(4)$ & $0.0363(11)$ \\
\hline H63 & 0.8743 & 0.5889 & 0.7446 & $0.044 *$ \\
\hline C64 & $0.7473(3)$ & $0.55957(15)$ & $0.7021(3)$ & $0.0298(10)$ \\
\hline C65 & $0.7705(3)$ & $0.52067(16)$ & $0.6569(3)$ & $0.0320(10)$ \\
\hline C66 & $0.8480(3)$ & $0.51969(17)$ & $0.6315(4)$ & $0.0346(11)$ \\
\hline H66 & 0.8867 & 0.5445 & 0.6430 & $0.041^{*}$ \\
\hline C67 & $0.8690(3)$ & $0.48300(19)$ & $0.5900(4)$ & $0.0390(12)$ \\
\hline H67 & 0.9216 & 0.4828 & 0.5728 & $0.047^{*}$ \\
\hline C68 & $0.8134(4)$ & $0.44667(18)$ & $0.5735(4)$ & $0.0409(12)$ \\
\hline H68 & 0.8281 & 0.4215 & 0.5450 & $0.049^{*}$ \\
\hline C69 & $0.7366(3)$ & $0.44671(18)$ & $0.5982(4)$ & $0.0389(12)$ \\
\hline H69 & 0.6988 & 0.4216 & 0.5867 & $0.047^{*}$ \\
\hline $\mathrm{C} 70$ & $0.7146(3)$ & $0.48316(16)$ & $0.6398(4)$ & $0.0332(11)$ \\
\hline N1 & $0.3219(3)$ & $0.36493(12)$ & $0.7882(3)$ & $0.0321(9)$ \\
\hline N2 & $0.4789(3)$ & $0.37504(13)$ & $0.7289(3)$ & $0.0339(9)$ \\
\hline N3 & $0.1712(3)$ & $0.39220(13)$ & $0.5053(3)$ & $0.0316(9)$ \\
\hline
\end{tabular}




$\begin{array}{lllll}\text { N4 } & 0.3296(3) & 0.41530(13) & 0.5315(3) & 0.0290(8) \\ \text { N5 } & 0.3158(3) & 0.43671(13) & 0.9063(3) & 0.0337(9) \\ \text { N6 } & 0.4215(3) & 0.47684(11) & 0.8493(3) & 0.0288(8) \\ \text { N7 } & 0.1558(3) & 0.46191(15) & 0.6258(3) & 0.0364(10) \\ \text { N8 } & 0.3047(3) & 0.52333(14) & 0.6565(3) & 0.0359(9) \\ \text { N9 } & 0.5880(3) & 0.45401(13) & 0.6849(3) & 0.0346(9) \\ \text { N10 } & 0.5047(3) & 0.52610(13) & 0.6498(3) & 0.0378(10) \\ \text { O1 } & 0.2406(3) & 0.35856(11) & 0.8007(3) & 0.0397(8) \\ \text { O2 } & 0.4437(2) & 0.41270(10) & 0.7481(3) & 0.0329(7) \\ \text { O3 } & 0.0833(2) & 0.38339(12) & 0.4931(3) & 0.0436(9) \\ \text { O4 } & 0.4102(2) & 0.43359(11) & 0.5495(2) & 0.0319(7) \\ \text { O5 } & 0.2580(3) & 0.41112(11) & 0.9310(3) & 0.0422(9) \\ \text { O6 } & 0.4795(2) & 0.49203(10) & 0.8119(3) & 0.0324(7) \\ \text { O7 } & 0.0898(3) & 0.43277(13) & 0.6260(3) & 0.0448(9) \\ \text { O8 } & 0.3220(2) & 0.48291(11) & 0.6323(3) & 0.0347(8) \\ \text { O9 } & 0.6191(2) & 0.41229(11) & 0.6839(3) & 0.0373(8) \\ \text { H1A } & 0.582(4) & 0.3945(14) & 0.694(5) & 0.056^{*} \\ \text { O10 } & 0.4608(3) & 0.56409(12) & 0.6526(3) & 0.0463(9) \\ \text { H2A } & 0.395(5) & 0.5572(5) & 0.654(5) & 0.069^{*} \\ \text { Ni1 } & 0.37718(6) & 0.41976(2) & 0.82564(6) & 0.0306(2) \\ \text { Ni2 } & 0.23963(5) & 0.44031(3) & 0.57163(6) & 0.0301(2) \\ \text { Ni3 } & 0.45817(5) & 0.46682(2) & 0.67739(6) & 0.0277(2) \\ \text { F1 } & 0.3252(3) & 0.34124(11) & 0.9649(3) & 0.0556(9) \\ \text { F2 } & 0.1678(3) & 0.35125(12) & 0.9084(3) & 0.0601(10) \\ \text { F3 } & -0.0369(2) & 0.39118(12) & 0.5394(3) & 0.0613(10) \\ \text { F4 } & 0.0009(2) & 0.44772(11) & 0.4612(3) & 0.0506(8) \\ \text { B1 } & 0.2493(5) & 0.3650(2) & 0.9034(6) & 0.0486(16) \\ \text { B2 } & 0.0335(4) & 0.4143(2) & 0.5275(6) & 0.0437(15)\end{array}$

Atomic displacement parameters $\left(\AA^{2}\right)$

\begin{tabular}{lllllll}
\hline & $U^{11}$ & $U^{22}$ & $U^{33}$ & $U^{12}$ & $U^{13}$ & $U^{23}$ \\
\hline $\mathrm{C} 1$ & $0.044(3)$ & $0.021(2)$ & $0.029(2)$ & $0.0022(19)$ & $0.011(2)$ & $0.0065(19)$ \\
$\mathrm{C} 2$ & $0.042(3)$ & $0.026(2)$ & $0.030(3)$ & $0.002(2)$ & $0.011(2)$ & $0.004(2)$ \\
$\mathrm{C} 3$ & $0.052(3)$ & $0.023(2)$ & $0.028(2)$ & $0.003(2)$ & $0.014(2)$ & $0.000(2)$ \\
$\mathrm{C} 4$ & $0.051(3)$ & $0.030(3)$ & $0.042(3)$ & $0.005(2)$ & $0.013(2)$ & $-0.002(2)$ \\
$\mathrm{C} 5$ & $0.058(3)$ & $0.035(3)$ & $0.052(4)$ & $0.010(3)$ & $0.015(3)$ & $-0.004(3)$ \\
C6 & $0.064(4)$ & $0.029(3)$ & $0.055(4)$ & $0.017(3)$ & $0.022(3)$ & $-0.001(3)$ \\
C7 & $0.068(4)$ & $0.024(3)$ & $0.038(3)$ & $0.001(2)$ & $0.012(3)$ & $-0.002(2)$ \\
C8 & $0.061(3)$ & $0.016(2)$ & $0.029(3)$ & $0.001(2)$ & $0.015(2)$ & $0.0017(19)$ \\
C9 & $0.058(3)$ & $0.020(2)$ & $0.028(2)$ & $-0.002(2)$ & $0.014(2)$ & $0.006(2)$ \\
C10 & $0.065(3)$ & $0.019(2)$ & $0.032(3)$ & $-0.006(2)$ & $0.016(2)$ & $0.002(2)$ \\
C11 & $0.059(3)$ & $0.033(3)$ & $0.035(3)$ & $-0.016(2)$ & $0.014(2)$ & $0.001(2)$ \\
C12 & $0.054(3)$ & $0.036(3)$ & $0.036(3)$ & $-0.010(2)$ & $0.015(2)$ & $0.002(2)$ \\
C13 & $0.049(3)$ & $0.028(3)$ & $0.031(3)$ & $-0.005(2)$ & $0.015(2)$ & $0.003(2)$ \\
C14 & $0.049(3)$ & $0.023(2)$ & $0.024(2)$ & $-0.006(2)$ & $0.010(2)$ & $0.0060(19)$ \\
C15 & $0.036(2)$ & $0.032(3)$ & $0.023(2)$ & $0.0051(19)$ & $0.0079(18)$ & $0.006(2)$ \\
C16 & $0.041(2)$ & $0.026(2)$ & $0.025(2)$ & $0.0059(19)$ & $0.0159(19)$ & $0.0028(19)$
\end{tabular}




\begin{tabular}{|c|c|c|c|c|c|c|}
\hline $\mathrm{C} 17$ & $0.041(2)$ & $0.029(2)$ & $0.022(2)$ & $0.0046(19)$ & $0.0133(19)$ & $0.0010(19)$ \\
\hline C18 & $0.039(2)$ & $0.034(3)$ & $0.032(3)$ & $0.001(2)$ & $0.013(2)$ & $-0.002(2)$ \\
\hline C19 & $0.041(3)$ & $0.041(3)$ & 0.049 (3) & $0.006(2)$ & $0.016(2)$ & $-0.007(3)$ \\
\hline $\mathrm{C} 20$ & $0.050(3)$ & $0.041(3)$ & 0.050 & $0.011(2)$ & 0.018 & -0.010 \\
\hline $\mathrm{C} 21$ & $0.048(3)$ & $0.033(3)$ & $0.044(3)$ & $0.001(2)$ & $0.015(2)$ & $-0.010(2)$ \\
\hline $\mathrm{C} 22$ & $0.045(3)$ & $0.030(3)$ & $0.025(2)$ & $0.002(2)$ & $0.015(2)$ & $-0.002(2)$ \\
\hline $\mathrm{C} 23$ & $0.048(3)$ & $0.029(3)$ & $0.026(2)$ & $0.000(2)$ & $0.016(2)$ & $0.003(2)$ \\
\hline $\mathrm{C} 24$ & $0.054(3)$ & $0.038(3)$ & 0.040 & $-0.005(2)$ & $0.023(3)$ & $-0.009(2)$ \\
\hline $\mathrm{C} 25$ & $0.061(3)$ & $0.042(3)$ & 0.038 & $-0.013(3)$ & $0.021(3)$ & -0.014 \\
\hline $\mathrm{C} 26$ & $0.050(3)$ & 0.049 (3) & 0.033 & -0.011 & $0.012(2)$ & -0.005 \\
\hline $\mathrm{C} 27$ & $0.043(3)$ & $0.037(3)$ & 0.033 & $0.002(2)$ & $0.013(2)$ & $0.004(2)$ \\
\hline $\mathrm{C} 28$ & $0.042(3)$ & $0.029(2)$ & $0.028(2)$ & $0.002(2)$ & $0.010(2)$ & $0.008(2)$ \\
\hline $\mathrm{C} 29$ & $0.045(3)$ & $0.023(2)$ & $0.026(2)$ & $0.0069(19)$ & $0.011(2)$ & $0.0031(19)$ \\
\hline $\mathrm{C} 30$ & $0.042(2)$ & $0.024(2)$ & $0.025(2)$ & $0.0043(19)$ & 0.0094 (19) & $0.0014(19)$ \\
\hline C31 & $0.048(3)$ & $0.021(2)$ & $0.021(2)$ & 0.0047 (19) & $0.0066(19)$ & $0.0026(19)$ \\
\hline $\mathrm{C} 32$ & $0.058(3)$ & $0.025(2)$ & 0.030 & $0.006(2)$ & $0.014(2)$ & $-0.001(2)$ \\
\hline C33 & $0.072(4)$ & $0.023(3)$ & 0.039 & $-0.004(2)$ & $0.021(3)$ & $-0.001(2)$ \\
\hline C34 & $0.095(5)$ & $0.020(3)$ & $0.051(4)$ & -0.003 & $0.027(3)$ & -0.009 \\
\hline $\mathrm{C} 35$ & $0.075(4)$ & 0.028 & $0.033(3)$ & $0.006(3)$ & $0.020(3)$ & $-0.006(2)$ \\
\hline $\mathrm{C} 36$ & 0.050 & $0.024(2)$ & $0.023(2)$ & $0.003(2)$ & 0.009 (2) & -0.0012 \\
\hline $\mathrm{C} 37$ & $0.045(3)$ & $0.032(3)$ & $0.021(2)$ & 0.009 (2) & $0.002(2)$ & $0.001(2)$ \\
\hline C38 & $0.053(3)$ & $0.038(3)$ & 0.028 & 0.009 (2) & $0.008(2)$ & $-0.009(2)$ \\
\hline C39 & $0.065(4)$ & $0.054(4)$ & 0.030 & 0.014 & $0.018(3)$ & -0.008 \\
\hline $\mathrm{C} 40$ & $0.054(3)$ & $0.054(4)$ & $0.023(2)$ & $0.011(3)$ & $0.011(2)$ & $0.004(2)$ \\
\hline $\mathrm{C} 41$ & $0.046(3)$ & $0.031(3)$ & 0.033 & $0.004(2)$ & $0.013(2)$ & $0.004(2)$ \\
\hline $\mathrm{C} 42$ & $0.052(3)$ & $0.028(3)$ & 0.019 (2) & $0.010(2)$ & $0.006(2)$ & $0.0008(19)$ \\
\hline $\mathrm{C} 43$ & $0.032(2)$ & $0.056(3)$ & $0.026(2)$ & $0.014(2)$ & $0.0044(19)$ & $-0.007(2)$ \\
\hline $\mathrm{C} 44$ & $0.032(2)$ & $0.046(3)$ & $0.031(3)$ & $0.011(2)$ & $0.003(2)$ & $-0.010(2)$ \\
\hline $\mathrm{C} 45$ & $0.036(3)$ & $0.057(4)$ & 0.045 & 0.017 (3) & $-0.002(2)$ & -0.021 \\
\hline $\mathrm{C} 46$ & $0.043(3)$ & $0.039(3)$ & $0.074(5)$ & 0.008 & $0.002(3)$ & -0.024 \\
\hline $\mathrm{C} 47$ & $0.052(4)$ & $0.056(4)$ & $0.103(6)$ & 0.009 (3) & $0.006(4)$ & $-0.036(4)$ \\
\hline $\mathrm{C} 48$ & $0.054(4)$ & $0.070(5)$ & $0.123(8)$ & 0.019 (4) & -0.005 & $-0.062(5)$ \\
\hline $\mathrm{C} 49$ & $0.053(4)$ & $0.093(6)$ & $0.082(6)$ & $0.024(4)$ & $0.001(4)$ & $-0.048(5)$ \\
\hline $\mathrm{C} 50$ & $0.037(3)$ & $0.080(5)$ & $0.048(4)$ & $0.018(3)$ & $-0.003(3)$ & $-0.034(4)$ \\
\hline $\mathrm{C} 51$ & $0.043(3)$ & $0.104(6)$ & 0.028 & $0.023(3)$ & $0.002(2)$ & $-0.024(3)$ \\
\hline $\mathrm{C} 52$ & $0.045(3)$ & $0.129(7)$ & $0.036(3)$ & $0.022(4)$ & $0.005(3)$ & $-0.028(4)$ \\
\hline $\mathrm{C} 53$ & $0.041(3)$ & $0.186(11)$ & $0.031(3)$ & $0.028(5)$ & 0.007 (3) & $-0.022(5)$ \\
\hline $\mathrm{C} 54$ & 0.045 & $0.155(9)$ & 0.028 & 0.007 (4) & $0.009(3)$ & -0.001 \\
\hline C55 & 0.037 & $0.115(6)$ & 0.025 & $0.006(3)$ & $0.007(2)$ & -0.010 \\
\hline $\mathrm{C} 56$ & $0.031(2)$ & $0.087(5)$ & $0.027(3)$ & 0.013 & $0.006(2)$ & $-0.012(3)$ \\
\hline $\mathrm{C} 57$ & $0.034(2)$ & $0.031(3)$ & $0.027(2)$ & $0.0035(19)$ & $0.0096(19)$ & $-0.003(2)$ \\
\hline $\mathrm{C} 58$ & $0.037(2)$ & $0.034(3)$ & $0.026(2)$ & $0.000(2)$ & $0.0141(19)$ & $-0.002(2)$ \\
\hline $\mathrm{C} 59$ & $0.041(2)$ & $0.027(2)$ & $0.025(2)$ & $0.0019(19)$ & 0.0137 (19) & $0.0026(19)$ \\
\hline $\mathrm{C} 60$ & $0.054(3)$ & $0.032(3)$ & $0.037(3)$ & $-0.002(2)$ & $0.023(2)$ & $0.001(2)$ \\
\hline C61 & 0.065 & $0.031(3)$ & $0.048(3)$ & $0.000(3)$ & $0.028(3)$ & $-0.007(3)$ \\
\hline C62 & $0.061(3)$ & $0.027(3)$ & $0.042(3)$ & $-0.012(2)$ & 0.018 & $-0.004(2)$ \\
\hline C63 & 0.044 & 0.034 & $0.030(3)$ & $-0.004(2)$ & $0.012(2)$ & $0.005(2)$ \\
\hline C64 & $0.041(2)$ & $0.028(2)$ & $0.018(2)$ & $0.0001(19)$ & $0.0091(18)$ & $0.0051(19)$ \\
\hline
\end{tabular}




\begin{tabular}{|c|c|c|c|c|c|c|}
\hline C65 & $0.041(2)$ & $0.031(3)$ & $0.022(2)$ & $0.001(2)$ & $0.0104(19)$ & $0.0010(19)$ \\
\hline C66 & $0.037(2)$ & $0.037(3)$ & $0.028(2)$ & $0.000(2)$ & $0.0108(19)$ & $0.006(2)$ \\
\hline C67 & $0.033(2)$ & $0.052(3)$ & $0.031(3)$ & $0.009(2)$ & $0.011(2)$ & $0.005(2)$ \\
\hline C68 & $0.044(3)$ & $0.042(3)$ & $0.038(3)$ & $0.002(2)$ & $0.017(2)$ & $-0.011(2)$ \\
\hline C69 & $0.039(3)$ & $0.041(3)$ & $0.036(3)$ & $-0.004(2)$ & $0.013(2)$ & $-0.009(2)$ \\
\hline C70 & $0.032(2)$ & $0.034(3)$ & $0.030(2)$ & $-0.0023(19)$ & $0.0083(19)$ & -0.003 \\
\hline N1 & $0.042(2)$ & $0.0176(18)$ & $0.039(2)$ & $-0.0029(16)$ & $0.0183(18)$ & $0.0038(17)$ \\
\hline $\mathrm{N} 2$ & $0.047(2)$ & $0.020(2)$ & $0.034(2)$ & $0.0032(17)$ & $0.0156(18)$ & $-0.0009(17)$ \\
\hline N3 & $0.0329(19)$ & $0.029(2)$ & $0.032(2)$ & $-0.0009(16)$ & $0.0119(17)$ & $0.0026(17)$ \\
\hline N4 & $0.0354(19)$ & $0.027(2)$ & $0.026(2)$ & $0.0023(16)$ & $0.0130(16)$ & $0.0018(16)$ \\
\hline N5 & $0.053(2)$ & $0.0196(19)$ & $0.032(2)$ & $0.0056(17)$ & $0.0206(19)$ & $0.0067(17)$ \\
\hline N6 & $0.038(2)$ & $0.0146(17)$ & $0.032(2)$ & $-0.0032(15)$ & $0.0109(16)$ & $0.0013(16)$ \\
\hline N7 & $0.034(2)$ & $0.046(3)$ & $0.029(2)$ & $0.0027(18)$ & $0.0125(17)$ & $0.0015(19)$ \\
\hline N8 & $0.038(2)$ & $0.032(2)$ & $0.033(2)$ & $0.0069(17)$ & $0.0086(17)$ & $-0.0043(18)$ \\
\hline N9 & $0.042(2)$ & $0.027(2)$ & $0.036(2)$ & $-0.0039(17)$ & $0.0156(18)$ & $-0.0092(18)$ \\
\hline N10 & $0.052(2)$ & $0.025(2)$ & $0.040(2)$ & $0.0038(18)$ & $0.021(2)$ & $-0.0010(19)$ \\
\hline $\mathrm{O} 1$ & $0.049(2)$ & $0.0295(18)$ & $0.046(2)$ & $-0.0043(15)$ & $0.0243(17)$ & $-0.0047(16)$ \\
\hline $\mathrm{O} 2$ & $0.055(2)$ & $0.0144(15)$ & $0.0378(19)$ & $0.0011(13)$ & $0.0274(16)$ & $0.0017(14)$ \\
\hline $\mathrm{O} 3$ & $0.0344(18)$ & $0.039(2)$ & $0.058(2)$ & $-0.0016(15)$ & $0.0185(17)$ & $-0.0068(19)$ \\
\hline $\mathrm{O} 4$ & $0.0332(16)$ & $0.0310(17)$ & $0.0326(18)$ & $-0.0019(13)$ & $0.0137(14)$ & $-0.0042(15)$ \\
\hline O5 & $0.064(2)$ & $0.0268(18)$ & $0.047(2)$ & $-0.0021(16)$ & $0.034(2)$ & $0.0006(17)$ \\
\hline O6 & $0.0429(18)$ & $0.0201(15)$ & $0.0347(18)$ & $-0.0032(13)$ & $0.0155(15)$ & $-0.0033(14)$ \\
\hline $\mathrm{O} 7$ & $0.043(2)$ & $0.054(2)$ & $0.043(2)$ & $-0.0077(17)$ & $0.0235(17)$ & $-0.0037(19)$ \\
\hline O8 & $0.0345(16)$ & $0.0273(17)$ & $0.041(2)$ & $0.0049(13)$ & $0.0123(15)$ & $-0.0044(15)$ \\
\hline O9 & $0.0384(18)$ & $0.0263(17)$ & $0.048(2)$ & $0.0018(14)$ & $0.0167(16)$ & $-0.0074(16)$ \\
\hline $\mathrm{O} 10$ & $0.057(2)$ & $0.0255(18)$ & $0.059(3)$ & $0.0003(16)$ & $0.025(2)$ & $0.0035(18)$ \\
\hline Ni1 & $0.0451(5)$ & $0.0169(4)$ & $0.0317(4)$ & $0.0006(3)$ & $0.0168(4)$ & $0.0009(3)$ \\
\hline $\mathrm{Ni} 2$ & $0.0342(4)$ & $0.0274(4)$ & $0.0293(4)$ & $0.0031(3)$ & $0.0127(3)$ & $-0.0014(3)$ \\
\hline $\mathrm{Ni3}$ & $0.0348(4)$ & $0.0182(4)$ & $0.0311(4)$ & $0.0013(3)$ & $0.0137(3)$ & $0.0008(3)$ \\
\hline F1 & $0.071(2)$ & $0.0393(18)$ & $0.058(2)$ & $0.0011(16)$ & $0.0272(18)$ & $0.0146(16)$ \\
\hline $\mathrm{F} 2$ & $0.072(2)$ & $0.048(2)$ & $0.072(3)$ & $-0.0111(17)$ & $0.041(2)$ & $-0.0011(19)$ \\
\hline F3 & $0.053(2)$ & $0.056(2)$ & $0.083(3)$ & $-0.0035(16)$ & $0.0350(19)$ & $-0.004(2)$ \\
\hline $\mathrm{F} 4$ & $0.0507(18)$ & $0.0436(19)$ & $0.049(2)$ & $0.0051(15)$ & $0.0097(15)$ & $-0.0025(16)$ \\
\hline B1 & $0.061(4)$ & $0.037(4)$ & $0.051(4)$ & -0.002 & $0.025(3)$ & $-0.001(3)$ \\
\hline B2 & 0.045 & $0.035(3)$ & $0.059(4)$ & -0.005 & $0.028(3)$ & -0.003 \\
\hline
\end{tabular}

Geometric parameters $\left(\AA,{ }^{o}\right)$

\begin{tabular}{llll}
\hline $\mathrm{C} 1-\mathrm{N} 1$ & $1.307(6)$ & $\mathrm{C} 44-\mathrm{C} 45$ & $1.485(8)$ \\
$\mathrm{C} 1-\mathrm{C} 2$ & $1.475(7)$ & $\mathrm{C} 45-\mathrm{C} 50$ & $1.385(9)$ \\
$\mathrm{C} 1-\mathrm{C} 14$ & $1.482(7)$ & $\mathrm{C} 45-\mathrm{C} 46$ & $1.399(10)$ \\
$\mathrm{C} 2-\mathrm{N} 2$ & $1.291(6)$ & $\mathrm{C} 46-\mathrm{C} 47$ & $1.388(9)$ \\
$\mathrm{C} 2-\mathrm{C} 3$ & $1.470(7)$ & $\mathrm{C} 46-\mathrm{H} 46$ & 0.9500 \\
$\mathrm{C} 3-\mathrm{C} 4$ & $1.394(7)$ & $\mathrm{C} 47-\mathrm{C} 48$ & $1.392(12)$ \\
$\mathrm{C} 3-\mathrm{C} 8$ & $1.418(7)$ & $\mathrm{C} 47-\mathrm{H} 47$ & 0.9500 \\
$\mathrm{C} 4-\mathrm{C} 5$ & $1.394(7)$ & $\mathrm{C} 48-\mathrm{C} 49$ & $1.375(14)$ \\
$\mathrm{C} 4-\mathrm{H} 4$ & 0.9500 & $\mathrm{C} 48-\mathrm{H} 48$ & 0.9500 \\
$\mathrm{C} 5-\mathrm{C} 6$ & $1.396(9)$ & $\mathrm{C} 49-\mathrm{C} 50$ & $1.413(10)$
\end{tabular}




\begin{tabular}{|c|c|c|c|}
\hline $\mathrm{C} 5-\mathrm{H} 5$ & 0.9500 & $\mathrm{C} 49-\mathrm{H} 49$ & 0.9500 \\
\hline $\mathrm{C} 6-\mathrm{C} 7$ & $1.367(8)$ & $\mathrm{C} 50-\mathrm{C} 51$ & $1.464(11)$ \\
\hline $\mathrm{C} 6-\mathrm{H} 6$ & 0.9500 & $\mathrm{C} 51-\mathrm{C} 52$ & $1.403(9)$ \\
\hline $\mathrm{C} 7-\mathrm{C} 8$ & $1.400(7)$ & C51-C56 & $1.405(10)$ \\
\hline $\mathrm{C} 7-\mathrm{H} 7$ & 0.9500 & $\mathrm{C} 52-\mathrm{C} 53$ & $1.364(13)$ \\
\hline $\mathrm{C} 8-\mathrm{C} 9$ & $1.458(8)$ & C52-H52 & 0.9500 \\
\hline $\mathrm{C} 9-\mathrm{C} 14$ & $1.410(7)$ & $\mathrm{C} 53-\mathrm{C} 54$ & $1.395(13)$ \\
\hline $\mathrm{C} 9-\mathrm{C} 10$ & $1.417(7)$ & C53-H53 & 0.9500 \\
\hline $\mathrm{C} 10-\mathrm{C} 11$ & $1.369(8)$ & $\mathrm{C} 54-\mathrm{C} 55$ & $1.399(9)$ \\
\hline $\mathrm{C} 10-\mathrm{H} 10$ & 0.9500 & C54-H54 & 0.9500 \\
\hline $\mathrm{C} 11-\mathrm{C} 12$ & $1.372(8)$ & C55-C56 & $1.404(10)$ \\
\hline C11-H11 & 0.9500 & C55-H55 & 0.9500 \\
\hline $\mathrm{C} 12-\mathrm{C} 13$ & $1.391(7)$ & $\mathrm{C} 57-\mathrm{N} 9$ & $1.273(6)$ \\
\hline $\mathrm{C} 12-\mathrm{H} 12$ & 0.9500 & $\mathrm{C} 57-\mathrm{C} 70$ & $1.466(7)$ \\
\hline $\mathrm{C} 13-\mathrm{C} 14$ & $1.395(7)$ & $\mathrm{C} 57-\mathrm{C} 58$ & $1.502(7)$ \\
\hline C13-H13 & 0.9500 & $\mathrm{C} 58-\mathrm{N} 10$ & $1.308(6)$ \\
\hline $\mathrm{C} 15-\mathrm{N} 3$ & $1.309(6)$ & $\mathrm{C} 58-\mathrm{C} 59$ & $1.470(7)$ \\
\hline $\mathrm{C} 15-\mathrm{C} 28$ & $1.464(7)$ & $\mathrm{C} 59-\mathrm{C} 60$ & $1.383(7)$ \\
\hline $\mathrm{C} 15-\mathrm{C} 16$ & $1.467(7)$ & C59-C64 & $1.407(7)$ \\
\hline $\mathrm{C} 16-\mathrm{N} 4$ & $1.314(6)$ & $\mathrm{C} 60-\mathrm{C} 61$ & $1.376(8)$ \\
\hline $\mathrm{C} 16-\mathrm{C} 17$ & $1.464(6)$ & $\mathrm{C} 60-\mathrm{H} 60$ & 0.9500 \\
\hline $\mathrm{C} 17-\mathrm{C} 18$ & $1.404(7)$ & C61-C62 & $1.377(8)$ \\
\hline $\mathrm{C} 17-\mathrm{C} 22$ & $1.416(7)$ & C61-H61 & 0.9500 \\
\hline $\mathrm{C} 18-\mathrm{C} 19$ & $1.391(7)$ & C62-C63 & $1.385(8)$ \\
\hline C18-H18 & 0.9500 & C62-H62 & 0.9500 \\
\hline $\mathrm{C} 19-\mathrm{C} 20$ & $1.384(8)$ & C63-C64 & $1.407(7)$ \\
\hline C19-H19 & 0.9500 & C63-H63 & 0.9500 \\
\hline $\mathrm{C} 20-\mathrm{C} 21$ & $1.376(8)$ & C64-C65 & $1.483(7)$ \\
\hline $\mathrm{C} 20-\mathrm{H} 20$ & 0.9500 & C65-C66 & $1.398(7)$ \\
\hline $\mathrm{C} 21-\mathrm{C} 22$ & $1.404(7)$ & $\mathrm{C} 65-\mathrm{C} 70$ & $1.414(7)$ \\
\hline $\mathrm{C} 21-\mathrm{H} 21$ & 0.9500 & C66-C67 & $1.384(7)$ \\
\hline $\mathrm{C} 22-\mathrm{C} 23$ & $1.465(7)$ & C66-H66 & 0.9500 \\
\hline $\mathrm{C} 23-\mathrm{C} 24$ & $1.410(7)$ & C67-C68 & $1.381(8)$ \\
\hline $\mathrm{C} 23-\mathrm{C} 28$ & $1.418(7)$ & C67-H67 & 0.9500 \\
\hline $\mathrm{C} 24-\mathrm{C} 25$ & $1.370(8)$ & C68-C69 & $1.382(7)$ \\
\hline $\mathrm{C} 24-\mathrm{H} 24$ & 0.9500 & C68-H68 & 0.9500 \\
\hline $\mathrm{C} 25-\mathrm{C} 26$ & $1.389(8)$ & $\mathrm{C} 69-\mathrm{C} 70$ & $1.385(7)$ \\
\hline $\mathrm{C} 25-\mathrm{H} 25$ & 0.9500 & C69-H69 & 0.9500 \\
\hline $\mathrm{C} 26-\mathrm{C} 27$ & $1.390(8)$ & $\mathrm{N} 1-\mathrm{O} 1$ & $1.367(5)$ \\
\hline $\mathrm{C} 26-\mathrm{H} 26$ & 0.9500 & $\mathrm{~N} 1-\mathrm{Ni1}$ & $1.883(4)$ \\
\hline $\mathrm{C} 27-\mathrm{C} 28$ & $1.399(7)$ & $\mathrm{N} 2-\mathrm{O} 2$ & $1.360(5)$ \\
\hline $\mathrm{C} 27-\mathrm{H} 27$ & 0.9500 & $\mathrm{~N} 3-\mathrm{O} 3$ & $1.346(5)$ \\
\hline $\mathrm{C} 29-\mathrm{N} 5$ & $1.315(6)$ & $\mathrm{N} 3-\mathrm{Ni} 2$ & $1.872(4)$ \\
\hline $\mathrm{C} 29-\mathrm{C} 30$ & $1.469(7)$ & $\mathrm{N} 4-\mathrm{O} 4$ & $1.312(5)$ \\
\hline $\mathrm{C} 29-\mathrm{C} 42$ & $1.476(7)$ & $\mathrm{N} 4-\mathrm{Ni} 2$ & $1.887(4)$ \\
\hline $\mathrm{C} 30-\mathrm{N} 6$ & $1.304(6)$ & N5-O5 & $1.350(5)$ \\
\hline $\mathrm{C} 30-\mathrm{C} 31$ & $1.460(7)$ & $\mathrm{N} 5-\mathrm{Ni} 1$ & $1.865(4)$ \\
\hline $\mathrm{C} 31-\mathrm{C} 32$ & $1.398(8)$ & $\mathrm{N} 6-\mathrm{O} 6$ & $1.314(5)$ \\
\hline
\end{tabular}




\begin{tabular}{|c|c|c|c|}
\hline $\mathrm{C} 31-\mathrm{C} 36$ & $1.432(7)$ & $\mathrm{N} 6-\mathrm{Ni1}$ & $1.875(4)$ \\
\hline $\mathrm{C} 32-\mathrm{C} 33$ & $1.371(7)$ & $\mathrm{N} 7-\mathrm{O} 7$ & $1.370(6)$ \\
\hline $\mathrm{C} 32-\mathrm{H} 32$ & 0.9500 & $\mathrm{~N} 7-\mathrm{Ni} 2$ & $1.897(4)$ \\
\hline $\mathrm{C} 33-\mathrm{C} 34$ & $1.379(8)$ & $\mathrm{N} 8-\mathrm{O} 8$ & $1.352(5)$ \\
\hline C $33-\mathrm{H} 33$ & 0.9500 & N9-O9 & $1.378(5)$ \\
\hline $\mathrm{C} 34-\mathrm{C} 35$ & $1.361(9)$ & $\mathrm{N} 9-\mathrm{Ni3}$ & 2.029 \\
\hline C34-H34 & 0.9500 & $\mathrm{~N} 10-\mathrm{O} 10$ & $1.367(5)$ \\
\hline $\mathrm{C} 35-\mathrm{C} 36$ & $1.393(7)$ & $\mathrm{N} 10-\mathrm{Ni3}$ & $2.064(4)$ \\
\hline C $35-\mathrm{H} 35$ & 0.9500 & $\mathrm{O} 1-\mathrm{B} 1$ & $1.480(8)$ \\
\hline $\mathrm{C} 36-\mathrm{C} 37$ & $1.470(8)$ & $\mathrm{O} 2-\mathrm{Ni} 1$ & $1.829(3)$ \\
\hline $\mathrm{C} 37-\mathrm{C} 38$ & $1.397(7)$ & $\mathrm{O} 2-\mathrm{Ni} 3$ & 2.025 \\
\hline $\mathrm{C} 37-\mathrm{C} 42$ & $1.411(7)$ & $\mathrm{O} 3-\mathrm{B} 2$ & $1.440(7)$ \\
\hline $\mathrm{C} 38-\mathrm{C} 39$ & $1.368(9)$ & $\mathrm{O} 4-\mathrm{Ni3}$ & 2.022 \\
\hline C $38-\mathrm{H} 38$ & 0.9500 & $\mathrm{O} 5-\mathrm{B} 1$ & $1.471(8)$ \\
\hline $\mathrm{C} 39-\mathrm{C} 40$ & $1.379(9)$ & $\mathrm{O} 6-\mathrm{Ni3}$ & $2.036(3)$ \\
\hline C39-H39 & 0.9500 & $\mathrm{O} 7-\mathrm{B} 2$ & $1.493(8)$ \\
\hline $\mathrm{C} 40-\mathrm{C} 41$ & $1.390(8)$ & $\mathrm{O} 8-\mathrm{Ni} 2$ & $1.819(3)$ \\
\hline $\mathrm{C} 40-\mathrm{H} 40$ & 0.9500 & $\mathrm{O} 8-\mathrm{Ni} 3$ & $2.036(3)$ \\
\hline $\mathrm{C} 41-\mathrm{C} 42$ & $1.389(8)$ & $\mathrm{O} 9-\mathrm{H} 1 \mathrm{~A}$ & $0.85(7)$ \\
\hline $\mathrm{C} 41-\mathrm{H} 41$ & 0.9500 & $\mathrm{O} 10-\mathrm{H} 2 \mathrm{~A}$ & $1.06(7)$ \\
\hline $\mathrm{C} 43-\mathrm{N} 7$ & $1.298(7)$ & $\mathrm{F} 1-\mathrm{B} 1$ & $1.395(8)$ \\
\hline $\mathrm{C} 43-\mathrm{C} 56$ & $1.471(7)$ & $\mathrm{F} 2-\mathrm{B} 1$ & $1.372(8)$ \\
\hline $\mathrm{C} 43-\mathrm{C} 44$ & $1.481(8)$ & $\mathrm{F} 3-\mathrm{B} 2$ & $1.378(7)$ \\
\hline $\mathrm{C} 44-\mathrm{N} 8$ & $1.290(7)$ & $\mathrm{F} 4-\mathrm{B} 2$ & $1.377(8)$ \\
\hline $\mathrm{N} 1-\mathrm{C} 1-\mathrm{C} 2$ & $117.6(4)$ & $\mathrm{C} 48-\mathrm{C} 49-\mathrm{H} 49$ & 119.1 \\
\hline $\mathrm{N} 1-\mathrm{C} 1-\mathrm{C} 14$ & $126.3(5)$ & $\mathrm{C} 50-\mathrm{C} 49-\mathrm{H} 49$ & 119.1 \\
\hline $\mathrm{C} 2-\mathrm{C} 1-\mathrm{C} 14$ & $116.2(4)$ & $\mathrm{C} 45-\mathrm{C} 50-\mathrm{C} 49$ & $117.4(8)$ \\
\hline $\mathrm{N} 2-\mathrm{C} 2-\mathrm{C} 3$ & $115.7(5)$ & $\mathrm{C} 45-\mathrm{C} 50-\mathrm{C} 51$ & $119.8(6)$ \\
\hline $\mathrm{N} 2-\mathrm{C} 2-\mathrm{C} 1$ & $126.5(4)$ & $\mathrm{C} 49-\mathrm{C} 50-\mathrm{C} 51$ & $122.8(7)$ \\
\hline $\mathrm{C} 3-\mathrm{C} 2-\mathrm{C} 1$ & $117.8(4)$ & $\mathrm{C} 52-\mathrm{C} 51-\mathrm{C} 56$ & $119.2(8)$ \\
\hline $\mathrm{C} 4-\mathrm{C} 3-\mathrm{C} 8$ & $120.7(5)$ & $\mathrm{C} 52-\mathrm{C} 51-\mathrm{C} 50$ & $118.9(7)$ \\
\hline $\mathrm{C} 4-\mathrm{C} 3-\mathrm{C} 2$ & $120.7(5)$ & $\mathrm{C} 56-\mathrm{C} 51-\mathrm{C} 50$ & $121.9(6)$ \\
\hline $\mathrm{C} 8-\mathrm{C} 3-\mathrm{C} 2$ & $118.7(5)$ & $\mathrm{C} 53-\mathrm{C} 52-\mathrm{C} 51$ & $120.0(8)$ \\
\hline $\mathrm{C} 3-\mathrm{C} 4-\mathrm{C} 5$ & $120.3(5)$ & $\mathrm{C} 53-\mathrm{C} 52-\mathrm{H} 52$ & 120.0 \\
\hline $\mathrm{C} 3-\mathrm{C} 4-\mathrm{H} 4$ & 119.9 & $\mathrm{C} 51-\mathrm{C} 52-\mathrm{H} 52$ & 120.0 \\
\hline $\mathrm{C} 5-\mathrm{C} 4-\mathrm{H} 4$ & 119.9 & $\mathrm{C} 52-\mathrm{C} 53-\mathrm{C} 54$ & $121.7(7)$ \\
\hline $\mathrm{C} 4-\mathrm{C} 5-\mathrm{C} 6$ & $119.2(6)$ & $\mathrm{C} 52-\mathrm{C} 53-\mathrm{H} 53$ & 119.1 \\
\hline $\mathrm{C} 4-\mathrm{C} 5-\mathrm{H} 5$ & 120.4 & $\mathrm{C} 54-\mathrm{C} 53-\mathrm{H} 53$ & 119.1 \\
\hline $\mathrm{C} 6-\mathrm{C} 5-\mathrm{H} 5$ & 120.4 & $\mathrm{C} 53-\mathrm{C} 54-\mathrm{C} 55$ & $119.2(9)$ \\
\hline $\mathrm{C} 7-\mathrm{C} 6-\mathrm{C} 5$ & $120.6(5)$ & $\mathrm{C} 53-\mathrm{C} 54-\mathrm{H} 54$ & 120.4 \\
\hline $\mathrm{C} 7-\mathrm{C} 6-\mathrm{H} 6$ & 119.7 & $\mathrm{C} 55-\mathrm{C} 54-\mathrm{H} 54$ & 120.4 \\
\hline $\mathrm{C} 5-\mathrm{C} 6-\mathrm{H} 6$ & 119.7 & $\mathrm{C} 54-\mathrm{C} 55-\mathrm{C} 56$ & $119.5(8)$ \\
\hline $\mathrm{C} 6-\mathrm{C} 7-\mathrm{C} 8$ & $122.0(5)$ & C54-C55-H55 & 120.2 \\
\hline $\mathrm{C} 6-\mathrm{C} 7-\mathrm{H} 7$ & 119.0 & $\mathrm{C} 56-\mathrm{C} 55-\mathrm{H} 55$ & 120.2 \\
\hline $\mathrm{C} 8-\mathrm{C} 7-\mathrm{H} 7$ & 119.0 & $\mathrm{C} 55-\mathrm{C} 56-\mathrm{C} 51$ & $120.2(6)$ \\
\hline $\mathrm{C} 7-\mathrm{C} 8-\mathrm{C} 3$ & $117.3(5)$ & $\mathrm{C} 55-\mathrm{C} 56-\mathrm{C} 43$ & $120.3(6)$ \\
\hline $\mathrm{C} 7-\mathrm{C} 8-\mathrm{C} 9$ & $123.3(5)$ & $\mathrm{C} 51-\mathrm{C} 56-\mathrm{C} 43$ & $119.3(6)$ \\
\hline
\end{tabular}




\begin{tabular}{|c|c|}
\hline $\mathrm{C} 3-\mathrm{C} 8-\mathrm{C} 9$ & $119.4(4)$ \\
\hline $\mathrm{C} 14-\mathrm{C} 9-\mathrm{C} 10$ & $117.2(5)$ \\
\hline $\mathrm{C} 14-\mathrm{C} 9-\mathrm{C} 8$ & $121.8(4)$ \\
\hline $\mathrm{C} 10-\mathrm{C} 9-\mathrm{C} 8$ & $121.0(5)$ \\
\hline $\mathrm{C} 11-\mathrm{C} 10-\mathrm{C} 9$ & $121.5(5)$ \\
\hline $\mathrm{C} 11-\mathrm{C} 10-\mathrm{H} 10$ & 119.3 \\
\hline $\mathrm{C} 9-\mathrm{C} 10-\mathrm{H} 10$ & 119.3 \\
\hline $\mathrm{C} 10-\mathrm{C} 11-\mathrm{C} 12$ & $121.1(5)$ \\
\hline $\mathrm{C} 10-\mathrm{C} 11-\mathrm{H} 11$ & 119.4 \\
\hline $\mathrm{C} 12-\mathrm{C} 11-\mathrm{H} 11$ & 119.4 \\
\hline $\mathrm{C} 11-\mathrm{C} 12-\mathrm{C} 13$ & $119.0(5)$ \\
\hline $\mathrm{C} 11-\mathrm{C} 12-\mathrm{H} 12$ & 120.5 \\
\hline $\mathrm{C} 13-\mathrm{C} 12-\mathrm{H} 12$ & 120.5 \\
\hline $\mathrm{C} 12-\mathrm{C} 13-\mathrm{C} 14$ & $121.1(5)$ \\
\hline $\mathrm{C} 12-\mathrm{C} 13-\mathrm{H} 13$ & 119.5 \\
\hline $\mathrm{C} 14-\mathrm{C} 13-\mathrm{H} 13$ & 119.5 \\
\hline $\mathrm{C} 13-\mathrm{C} 14-\mathrm{C} 9$ & $120.0(5)$ \\
\hline $\mathrm{C} 13-\mathrm{C} 14-\mathrm{C} 1$ & $121.5(5)$ \\
\hline $\mathrm{C} 9-\mathrm{C} 14-\mathrm{C} 1$ & $118.3(5)$ \\
\hline $\mathrm{N} 3-\mathrm{C} 15-\mathrm{C} 28$ & $127.5(5)$ \\
\hline $\mathrm{N} 3-\mathrm{C} 15-\mathrm{C} 16$ & $112.3(4)$ \\
\hline $\mathrm{C} 28-\mathrm{C} 15-\mathrm{C} 16$ & $120.1(4)$ \\
\hline $\mathrm{N} 4-\mathrm{C} 16-\mathrm{C} 17$ & $127.5(4)$ \\
\hline $\mathrm{N} 4-\mathrm{C} 16-\mathrm{C} 15$ & $112.2(4)$ \\
\hline $\mathrm{C} 17-\mathrm{C} 16-\mathrm{C} 15$ & $120.2(4)$ \\
\hline $\mathrm{C} 18-\mathrm{C} 17-\mathrm{C} 22$ & $119.4(4)$ \\
\hline $\mathrm{C} 18-\mathrm{C} 17-\mathrm{C} 16$ & $122.8(5)$ \\
\hline $\mathrm{C} 22-\mathrm{C} 17-\mathrm{C} 16$ & $117.7(4)$ \\
\hline $\mathrm{C} 19-\mathrm{C} 18-\mathrm{C} 17$ & $121.0(5)$ \\
\hline $\mathrm{C} 19-\mathrm{C} 18-\mathrm{H} 18$ & 119.5 \\
\hline $\mathrm{C} 17-\mathrm{C} 18-\mathrm{H} 18$ & 119.5 \\
\hline $\mathrm{C} 20-\mathrm{C} 19-\mathrm{C} 18$ & $119.4(5)$ \\
\hline $\mathrm{C} 20-\mathrm{C} 19-\mathrm{H} 19$ & 120.3 \\
\hline $\mathrm{C} 18-\mathrm{C} 19-\mathrm{H} 19$ & 120.3 \\
\hline $\mathrm{C} 21-\mathrm{C} 20-\mathrm{C} 19$ & $120.4(5)$ \\
\hline $\mathrm{C} 21-\mathrm{C} 20-\mathrm{H} 20$ & 119.8 \\
\hline $\mathrm{C} 19-\mathrm{C} 20-\mathrm{H} 20$ & 119.8 \\
\hline $\mathrm{C} 20-\mathrm{C} 21-\mathrm{C} 22$ & $121.8(5)$ \\
\hline $\mathrm{C} 20-\mathrm{C} 21-\mathrm{H} 21$ & 119.1 \\
\hline $\mathrm{C} 22-\mathrm{C} 21-\mathrm{H} 21$ & 119.1 \\
\hline $\mathrm{C} 21-\mathrm{C} 22-\mathrm{C} 17$ & $117.9(5)$ \\
\hline $\mathrm{C} 21-\mathrm{C} 22-\mathrm{C} 23$ & $120.6(5)$ \\
\hline $\mathrm{C} 17-\mathrm{C} 22-\mathrm{C} 23$ & $121.3(4)$ \\
\hline $\mathrm{C} 24-\mathrm{C} 23-\mathrm{C} 28$ & $117.1(5)$ \\
\hline $\mathrm{C} 24-\mathrm{C} 23-\mathrm{C} 22$ & $121.3(5)$ \\
\hline $\mathrm{C} 28-\mathrm{C} 23-\mathrm{C} 22$ & $121.6(5)$ \\
\hline $\mathrm{C} 25-\mathrm{C} 24-\mathrm{C} 23$ & $122.7(5)$ \\
\hline $\mathrm{C} 25-\mathrm{C} 24$ & \\
\hline
\end{tabular}

$117.2(5)$

$121.8(4)$

$121.0(5)$

$121.5(5)$

119.3

$121.1(5)$

119.4

119.4

$119.0(5)$

120.5

$121.1(5)$

119.5

$120.0(5)$

$121.5(5)$

$118.3(5)$

$127.5(5)$

$112.3(4)$

$120.1(4)$

$127.5(4)$

120.2 (4)

$119.4(4)$

$122.8(5)$

$117.7(4)$

$121.0(5)$

119.5

119.5

$119.4(5)$

120.3

120.3

$120.4(5)$

119.8

$121.8(5)$

119.1

119.1

$117.9(5)$

$120.6(5)$

$121.3(4)$

$117.1(5)$

$121.3(5)$

$122.7(5)$

118.6
N9-C57-C70

N9-C57-C58

C $70-\mathrm{C} 57-\mathrm{C} 58$

N10-C58-C59

N10-C58-C57

C59-C58-C57

$\mathrm{C} 60-\mathrm{C} 59-\mathrm{C} 64$

$\mathrm{C} 60-\mathrm{C} 59-\mathrm{C} 58$

$\mathrm{C} 64-\mathrm{C} 59-\mathrm{C} 58$

$\mathrm{C} 61-\mathrm{C} 60-\mathrm{C} 59$

$\mathrm{C} 61-\mathrm{C} 60-\mathrm{H} 60$

$\mathrm{C} 59-\mathrm{C} 60-\mathrm{H} 60$

C60-C61-C62

C60- $661-\mathrm{H} 61$

C62-C61-H61

C61-C62-C63

C61-C62-H62

C63-C62-H62

C62-C63-C64

C62- $663-\mathrm{H} 63$

C64-C63-H63

C59-C64-C63

C59-C64-C65

C63-C64-C65

C66-C65-C70

C66-C65-C64

C70-C65-C64

C67-C66-C65

C67-C66-H66

C65-C66-H66

C68-C67-C66

C68-C67-H67

C66-C67-H67

C67-C68-C69

C67- $668-\mathrm{H} 68$

$\mathrm{C} 69-\mathrm{C} 68-\mathrm{H} 68$

C68-C69-C70

C68-C69-H69

C70- C69-H69

$\mathrm{C} 69-\mathrm{C} 70-\mathrm{C} 65$

$\mathrm{C} 69-\mathrm{C} 70-\mathrm{C} 57$

C65-C70-C57

$\mathrm{C} 1-\mathrm{N} 1-\mathrm{O} 1$

C1-N1-Ni1

O1-N1-Ni1

$\mathrm{C} 2-\mathrm{N} 2-\mathrm{O} 2$

$\mathrm{C} 15-\mathrm{N} 3-\mathrm{O} 3$

C15-N3-Ni2
$130.1(5)$

$113.1(4)$

$116.8(4)$

$131.6(4)$

$110.5(4)$

$117.4(4)$

120.4 (5)

121.9 (4)

117.5 (4)

120.5 (5)

119.8

119.8

120.3 (5)

119.8

119.8

$120.3(5)$

119.9

119.9

120.4 (5)

119.8

119.8

$118.1(5)$

$121.3(4)$

$120.6(4)$

$118.4(5)$

$121.5(4)$

$120.0(4)$

$120.8(5)$

119.6

119.6

120.1 (5)

119.9

119.9

120.3 (5)

119.9

119.9

120.4 (5)

119.8

119.8

$120.0(5)$

121.9 (4)

118.1 (4)

116.1 (4)

126.1 (3)

$115.8(3)$

117.3 (4)

117.4 (4)

$117.0(3)$ 


\begin{tabular}{|c|c|}
\hline $\mathrm{C} 23-\mathrm{C} 24-\mathrm{H} 24$ & 118.6 \\
\hline $\mathrm{C} 24-\mathrm{C} 25-\mathrm{C} 26$ & $119.7(5)$ \\
\hline $\mathrm{C} 24-\mathrm{C} 25-\mathrm{H} 25$ & 120.2 \\
\hline $\mathrm{C} 26-\mathrm{C} 25-\mathrm{H} 25$ & 120.2 \\
\hline $\mathrm{C} 25-\mathrm{C} 26-\mathrm{C} 27$ & $119.6(5)$ \\
\hline $\mathrm{C} 25-\mathrm{C} 26-\mathrm{H} 26$ & 120.2 \\
\hline $\mathrm{C} 27-\mathrm{C} 26-\mathrm{H} 26$ & 120.2 \\
\hline $\mathrm{C} 26-\mathrm{C} 27-\mathrm{C} 28$ & $121.1(5)$ \\
\hline $\mathrm{C} 26-\mathrm{C} 27-\mathrm{H} 27$ & 119.5 \\
\hline $\mathrm{C} 28-\mathrm{C} 27-\mathrm{H} 27$ & 119.5 \\
\hline $\mathrm{C} 27-\mathrm{C} 28-\mathrm{C} 23$ & $119.7(5)$ \\
\hline $\mathrm{C} 27-\mathrm{C} 28-\mathrm{C} 15$ & $123.0(5)$ \\
\hline $\mathrm{C} 23-\mathrm{C} 28-\mathrm{C} 15$ & $117.3(4)$ \\
\hline $\mathrm{N} 5-\mathrm{C} 29-\mathrm{C} 30$ & $112.0(4)$ \\
\hline $\mathrm{N} 5-\mathrm{C} 29-\mathrm{C} 42$ & $127.8(5)$ \\
\hline $\mathrm{C} 30-\mathrm{C} 29-\mathrm{C} 42$ & $120.3(4)$ \\
\hline $\mathrm{N} 6-\mathrm{C} 30-\mathrm{C} 31$ & $126.9(5)$ \\
\hline $\mathrm{N} 6-\mathrm{C} 30-\mathrm{C} 29$ & $112.3(4)$ \\
\hline $\mathrm{C} 31-\mathrm{C} 30-\mathrm{C} 29$ & $120.9(4)$ \\
\hline $\mathrm{C} 32-\mathrm{C} 31-\mathrm{C} 36$ & $118.8(4)$ \\
\hline $\mathrm{C} 32-\mathrm{C} 31-\mathrm{C} 30$ & $123.2(5)$ \\
\hline $\mathrm{C} 36-\mathrm{C} 31-\mathrm{C} 30$ & $118.0(5)$ \\
\hline $\mathrm{C} 33-\mathrm{C} 32-\mathrm{C} 31$ & $121.8(5)$ \\
\hline $\mathrm{C} 33-\mathrm{C} 32-\mathrm{H} 32$ & 119.1 \\
\hline $\mathrm{C} 31-\mathrm{C} 32-\mathrm{H} 32$ & 119.1 \\
\hline $\mathrm{C} 32-\mathrm{C} 33-\mathrm{C} 34$ & $119.7(6)$ \\
\hline $\mathrm{C} 32-\mathrm{C} 33-\mathrm{H} 33$ & 120.1 \\
\hline $\mathrm{C} 34-\mathrm{C} 33-\mathrm{H} 33$ & 120.1 \\
\hline $\mathrm{C} 35-\mathrm{C} 34-\mathrm{C} 33$ & $119.5(5)$ \\
\hline $\mathrm{C} 35-\mathrm{C} 34-\mathrm{H} 34$ & 120.2 \\
\hline $\mathrm{C} 33-\mathrm{C} 34-\mathrm{H} 34$ & 120.2 \\
\hline $\mathrm{C} 34-\mathrm{C} 35-\mathrm{C} 36$ & $123.6(5)$ \\
\hline C $34-\mathrm{C} 35-\mathrm{H} 35$ & 118.2 \\
\hline $\mathrm{C} 36-\mathrm{C} 35-\mathrm{H} 35$ & 118.2 \\
\hline $\mathrm{C} 35-\mathrm{C} 36-\mathrm{C} 31$ & $116.6(5)$ \\
\hline $\mathrm{C} 35-\mathrm{C} 36-\mathrm{C} 37$ & $122.7(5)$ \\
\hline $\mathrm{C} 31-\mathrm{C} 36-\mathrm{C} 37$ & $120.7(4)$ \\
\hline $\mathrm{C} 38-\mathrm{C} 37-\mathrm{C} 42$ & $118.0(5)$ \\
\hline $\mathrm{C} 38-\mathrm{C} 37-\mathrm{C} 36$ & $119.4(5)$ \\
\hline $\mathrm{C} 42-\mathrm{C} 37-\mathrm{C} 36$ & $122.6(5)$ \\
\hline $\mathrm{C} 39-\mathrm{C} 38-\mathrm{C} 37$ & $121.8(5)$ \\
\hline $\mathrm{C} 39-\mathrm{C} 38-\mathrm{H} 38$ & 119.1 \\
\hline $\mathrm{C} 37-\mathrm{C} 38-\mathrm{H} 38$ & 119.1 \\
\hline $\mathrm{C} 38-\mathrm{C} 39-\mathrm{C} 40$ & $120.3(5)$ \\
\hline C $38-\mathrm{C} 39-\mathrm{H} 39$ & 119.8 \\
\hline $\mathrm{C} 40-\mathrm{C} 39-\mathrm{H} 39$ & 119.8 \\
\hline $\mathrm{C} 39-\mathrm{C} 40-\mathrm{C} 41$ & $119.4(6)$ \\
\hline $\mathrm{C} 39-\mathrm{C} 40-\mathrm{H} 40$ & 120.3 \\
\hline
\end{tabular}

$125.6(3)$
$121.4(4)$
$122.3(3)$
$116.2(3)$
$118.5(4)$
$116.9(4)$
$124.6(3)$
$121.7(4)$
$116.8(3)$
$121.5(3)$
$116.4(4)$
$127.2(4)$
$114.4(3)$
$118.6(4)$
$117.6(4)$
$117.9(3)$
$122.1(3)$
$116.3(4)$
$115.7(3)$
$122.3(3)$
$113.0(4)$
$127.1(3)$
$117.6(3)$
$115.17(16)$
$118.8(4)$
$113.1(3)$
$119.5(4)$
$112.9(3)$
$114.4(4)$
$128.3(3)$
$115.4(3)$
$116.21(17)$
109.5
109.5
$170.30(16)$
$88.27(16)$
$82.07(18)$
$90.96(16)$
$98.53(18)$
$173.25(18)$
$170.42(16)$
$88.60(16)$
$81.88(17)$
$90.78(17)$
$98.56(19)$
$173.36(18)$
$89.28(14)$
$87.74(15)$




\begin{tabular}{|c|c|c|c|}
\hline $\mathrm{C} 41-\mathrm{C} 40-\mathrm{H} 40$ & 120.3 & $\mathrm{O} 2-\mathrm{Ni} 3-\mathrm{N} 9$ & $96.72(16)$ \\
\hline $\mathrm{C} 42-\mathrm{C} 41-\mathrm{C} 40$ & $120.9(5)$ & $\mathrm{O} 4-\mathrm{Ni} 3-\mathrm{O} 6$ & $165.12(14)$ \\
\hline $\mathrm{C} 42-\mathrm{C} 41-\mathrm{H} 41$ & 119.6 & $\mathrm{O} 2-\mathrm{Ni} 3-\mathrm{O} 6$ & $79.77(13)$ \\
\hline $\mathrm{C} 40-\mathrm{C} 41-\mathrm{H} 41$ & 119.6 & $\mathrm{~N} 9-\mathrm{Ni3}-\mathrm{O} 6$ & $103.43(16)$ \\
\hline $\mathrm{C} 41-\mathrm{C} 42-\mathrm{C} 37$ & $119.6(5)$ & $\mathrm{O} 4-\mathrm{Ni} 3-\mathrm{O} 8$ & $81.29(14)$ \\
\hline $\mathrm{C} 41-\mathrm{C} 42-\mathrm{C} 29$ & $123.0(5)$ & $\mathrm{O} 2-\mathrm{Ni} 3-\mathrm{O} 8$ & $93.48(15)$ \\
\hline $\mathrm{C} 37-\mathrm{C} 42-\mathrm{C} 29$ & $117.4(5)$ & $\mathrm{N} 9-\mathrm{Ni} 3-\mathrm{O} 8$ & $164.91(17)$ \\
\hline $\mathrm{N} 7-\mathrm{C} 43-\mathrm{C} 56$ & $127.1(6)$ & $\mathrm{O} 6-\mathrm{Ni3}-\mathrm{O} 8$ & $89.28(14)$ \\
\hline $\mathrm{N} 7-\mathrm{C} 43-\mathrm{C} 44$ & $117.3(5)$ & $\mathrm{O} 4-\mathrm{Ni3}-\mathrm{N} 10$ & $107.36(16)$ \\
\hline $\mathrm{C} 56-\mathrm{C} 43-\mathrm{C} 44$ & $115.5(5)$ & $\mathrm{O} 2-\mathrm{Ni} 3-\mathrm{N} 10$ & $161.43(17)$ \\
\hline $\mathrm{N} 8-\mathrm{C} 44-\mathrm{C} 43$ & $126.5(5)$ & N9-Ni3-N10 & $76.28(17)$ \\
\hline $\mathrm{N} 8-\mathrm{C} 44-\mathrm{C} 45$ & $114.4(5)$ & $\mathrm{O} 6-\mathrm{Ni3}-\mathrm{N} 10$ & $85.12(16)$ \\
\hline $\mathrm{C} 43-\mathrm{C} 44-\mathrm{C} 45$ & $119.1(5)$ & $\mathrm{O} 8-\mathrm{Ni} 3-\mathrm{N} 10$ & $97.08(16)$ \\
\hline $\mathrm{C} 50-\mathrm{C} 45-\mathrm{C} 46$ & $120.8(6)$ & $\mathrm{F} 2-\mathrm{B} 1-\mathrm{F} 1$ & $112.4(5)$ \\
\hline $\mathrm{C} 50-\mathrm{C} 45-\mathrm{C} 44$ & $118.7(7)$ & $\mathrm{F} 2-\mathrm{B} 1-\mathrm{O} 5$ & $105.9(5)$ \\
\hline $\mathrm{C} 46-\mathrm{C} 45-\mathrm{C} 44$ & $120.5(5)$ & $\mathrm{F} 1-\mathrm{B} 1-\mathrm{O} 5$ & $111.2(5)$ \\
\hline $\mathrm{C} 47-\mathrm{C} 46-\mathrm{C} 45$ & $121.0(7)$ & $\mathrm{F} 2-\mathrm{B} 1-\mathrm{O} 1$ & $106.8(5)$ \\
\hline $\mathrm{C} 47-\mathrm{C} 46-\mathrm{H} 46$ & 119.5 & $\mathrm{~F} 1-\mathrm{B} 1-\mathrm{O} 1$ & $108.5(5)$ \\
\hline $\mathrm{C} 45-\mathrm{C} 46-\mathrm{H} 46$ & 119.5 & $\mathrm{O} 5-\mathrm{B} 1-\mathrm{O} 1$ & $112.0(5)$ \\
\hline $\mathrm{C} 46-\mathrm{C} 47-\mathrm{C} 48$ & $118.5(9)$ & $\mathrm{F} 4-\mathrm{B} 2-\mathrm{F} 3$ & $112.3(5)$ \\
\hline $\mathrm{C} 46-\mathrm{C} 47-\mathrm{H} 47$ & 120.7 & $\mathrm{~F} 4-\mathrm{B} 2-\mathrm{O} 3$ & $110.4(5)$ \\
\hline $\mathrm{C} 48-\mathrm{C} 47-\mathrm{H} 47$ & 120.7 & $\mathrm{~F} 3-\mathrm{B} 2-\mathrm{O} 3$ & $105.4(5)$ \\
\hline $\mathrm{C} 49-\mathrm{C} 48-\mathrm{C} 47$ & $120.5(7)$ & $\mathrm{F} 4-\mathrm{B} 2-\mathrm{O} 7$ & $109.1(5)$ \\
\hline $\mathrm{C} 49-\mathrm{C} 48-\mathrm{H} 48$ & 119.7 & $\mathrm{~F} 3-\mathrm{B} 2-\mathrm{O} 7$ & $106.1(5)$ \\
\hline $\mathrm{C} 47-\mathrm{C} 48-\mathrm{H} 48$ & 119.7 & $\mathrm{O} 3-\mathrm{B} 2-\mathrm{O} 7$ & $113.4(5)$ \\
\hline $\mathrm{C} 48-\mathrm{C} 49-\mathrm{C} 50$ & $121.7(8)$ & & \\
\hline $\mathrm{N} 1-\mathrm{C} 1-\mathrm{C} 2-\mathrm{N} 2$ & $-33.6(7)$ & $\mathrm{C} 54-\mathrm{C} 55-\mathrm{C} 56-\mathrm{C} 43$ & $176.8(5)$ \\
\hline $\mathrm{C} 14-\mathrm{C} 1-\mathrm{C} 2-\mathrm{N} 2$ & $146.8(5)$ & $\mathrm{C} 52-\mathrm{C} 51-\mathrm{C} 56-\mathrm{C} 55$ & $-1.5(8)$ \\
\hline $\mathrm{N} 1-\mathrm{C} 1-\mathrm{C} 2-\mathrm{C} 3$ & $146.9(5)$ & $\mathrm{C} 50-\mathrm{C} 51-\mathrm{C} 56-\mathrm{C} 55$ & $176.8(5)$ \\
\hline $\mathrm{C} 14-\mathrm{C} 1-\mathrm{C} 2-\mathrm{C} 3$ & $-32.8(6)$ & $\mathrm{C} 52-\mathrm{C} 51-\mathrm{C} 56-\mathrm{C} 43$ & $-176.3(5)$ \\
\hline $\mathrm{N} 2-\mathrm{C} 2-\mathrm{C} 3-\mathrm{C} 4$ & $22.9(7)$ & $\mathrm{C} 50-\mathrm{C} 51-\mathrm{C} 56-\mathrm{C} 43$ & $2.0(8)$ \\
\hline $\mathrm{C} 1-\mathrm{C} 2-\mathrm{C} 3-\mathrm{C} 4$ & $-157.5(5)$ & $\mathrm{N} 7-\mathrm{C} 43-\mathrm{C} 56-\mathrm{C} 55$ & $24.5(8)$ \\
\hline $\mathrm{N} 2-\mathrm{C} 2-\mathrm{C} 3-\mathrm{C} 8$ & $-157.1(5)$ & $\mathrm{C} 44-\mathrm{C} 43-\mathrm{C} 56-\mathrm{C} 55$ & $-157.4(5)$ \\
\hline $\mathrm{C} 1-\mathrm{C} 2-\mathrm{C} 3-\mathrm{C} 8$ & $22.6(7)$ & $\mathrm{N} 7-\mathrm{C} 43-\mathrm{C} 56-\mathrm{C} 51$ & $-160.7(5)$ \\
\hline $\mathrm{C} 8-\mathrm{C} 3-\mathrm{C} 4-\mathrm{C} 5$ & $0.1(8)$ & $\mathrm{C} 44-\mathrm{C} 43-\mathrm{C} 56-\mathrm{C} 51$ & $17.4(7)$ \\
\hline $\mathrm{C} 2-\mathrm{C} 3-\mathrm{C} 4-\mathrm{C} 5$ & $-179.8(5)$ & N9-C $57-\mathrm{C} 58-\mathrm{N} 10$ & $-28.2(6)$ \\
\hline $\mathrm{C} 3-\mathrm{C} 4-\mathrm{C} 5-\mathrm{C} 6$ & $-0.2(9)$ & $\mathrm{C} 70-\mathrm{C} 57-\mathrm{C} 58-\mathrm{N} 10$ & $152.2(4)$ \\
\hline $\mathrm{C} 4-\mathrm{C} 5-\mathrm{C} 6-\mathrm{C} 7$ & $-0.2(10)$ & N9-C57-C58-C59 & $145.3(4)$ \\
\hline $\mathrm{C} 5-\mathrm{C} 6-\mathrm{C} 7-\mathrm{C} 8$ & $0.7(10)$ & $\mathrm{C} 70-\mathrm{C} 57-\mathrm{C} 58-\mathrm{C} 59$ & $-34.4(6)$ \\
\hline $\mathrm{C} 6-\mathrm{C} 7-\mathrm{C} 8-\mathrm{C} 3$ & $-0.7(8)$ & $\mathrm{N} 10-\mathrm{C} 58-\mathrm{C} 59-\mathrm{C} 60$ & $17.3(8)$ \\
\hline $\mathrm{C} 6-\mathrm{C} 7-\mathrm{C} 8-\mathrm{C} 9$ & $179.0(5)$ & $\mathrm{C} 57-\mathrm{C} 58-\mathrm{C} 59-\mathrm{C} 60$ & $-154.5(5)$ \\
\hline $\mathrm{C} 4-\mathrm{C} 3-\mathrm{C} 8-\mathrm{C} 7$ & $0.3(8)$ & $\mathrm{N} 10-\mathrm{C} 58-\mathrm{C} 59-\mathrm{C} 64$ & $-168.3(5)$ \\
\hline $\mathrm{C} 2-\mathrm{C} 3-\mathrm{C} 8-\mathrm{C} 7$ & $-179.7(5)$ & $\mathrm{C} 57-\mathrm{C} 58-\mathrm{C} 59-\mathrm{C} 64$ & $19.9(6)$ \\
\hline $\mathrm{C} 4-\mathrm{C} 3-\mathrm{C} 8-\mathrm{C} 9$ & $-179.5(5)$ & $\mathrm{C} 64-\mathrm{C} 59-\mathrm{C} 60-\mathrm{C} 61$ & $-1.7(8)$ \\
\hline $\mathrm{C} 2-\mathrm{C} 3-\mathrm{C} 8-\mathrm{C} 9$ & $0.5(7)$ & $\mathrm{C} 58-\mathrm{C} 59-\mathrm{C} 60-\mathrm{C} 61$ & $172.6(5)$ \\
\hline $\mathrm{C} 7-\mathrm{C} 8-\mathrm{C} 9-\mathrm{C} 14$ & $166.8(5)$ & $\mathrm{C} 59-\mathrm{C} 60-\mathrm{C} 61-\mathrm{C} 62$ & $1.4(9)$ \\
\hline $\mathrm{C} 3-\mathrm{C} 8-\mathrm{C} 9-\mathrm{C} 14$ & $-13.4(7)$ & $\mathrm{C} 60-\mathrm{C} 61-\mathrm{C} 62-\mathrm{C} 63$ & $-0.8(9)$ \\
\hline
\end{tabular}




\begin{tabular}{|c|c|}
\hline $\mathrm{C} 7-\mathrm{C} 8-\mathrm{C} 9-\mathrm{C} 10$ & $-15.0(8)$ \\
\hline $\mathrm{C} 3-\mathrm{C} 8-\mathrm{C} 9-\mathrm{C} 10$ & $164.8(5)$ \\
\hline $\mathrm{C} 14-\mathrm{C} 9-\mathrm{C} 10-\mathrm{C} 11$ & $-0.2(7)$ \\
\hline $\mathrm{C} 8-\mathrm{C} 9-\mathrm{C} 10-\mathrm{C} 11$ & $-178.5(5)$ \\
\hline $\mathrm{C} 9-\mathrm{C} 10-\mathrm{C} 11-\mathrm{C} 12$ & $1.3(8)$ \\
\hline $\mathrm{C} 10-\mathrm{C} 11-\mathrm{C} 12-\mathrm{C} 13$ & $-1.1(8)$ \\
\hline $\mathrm{C} 11-\mathrm{C} 12-\mathrm{C} 13-\mathrm{C} 14$ & $-0.1(8)$ \\
\hline $\mathrm{C} 12-\mathrm{C} 13-\mathrm{C} 14-\mathrm{C} 9$ & $1.2(7)$ \\
\hline $\mathrm{C} 12-\mathrm{C} 13-\mathrm{C} 14-\mathrm{C} 1$ & $175.8(5)$ \\
\hline $\mathrm{C} 10-\mathrm{C} 9-\mathrm{C} 14-\mathrm{C} 13$ & $-1.0(7)$ \\
\hline $\mathrm{C} 8-\mathrm{C} 9-\mathrm{C} 14-\mathrm{C} 13$ & $177.2(5)$ \\
\hline $\mathrm{C} 10-\mathrm{C} 9-\mathrm{C} 14-\mathrm{C} 1$ & $-175.8(4)$ \\
\hline $\mathrm{C} 8-\mathrm{C} 9-\mathrm{C} 14-\mathrm{C} 1$ & $2.5(7)$ \\
\hline $\mathrm{N} 1-\mathrm{C} 1-\mathrm{C} 14-\mathrm{C} 13$ & $26.0(7)$ \\
\hline $\mathrm{C} 2-\mathrm{C} 1-\mathrm{C} 14-\mathrm{C} 13$ & $-154.4(5)$ \\
\hline $\mathrm{N} 1-\mathrm{C} 1-\mathrm{C} 14-\mathrm{C} 9$ & $-159.3(5)$ \\
\hline $\mathrm{C} 2-\mathrm{C} 1-\mathrm{C} 14-\mathrm{C} 9$ & $20.3(6)$ \\
\hline $\mathrm{N} 3-\mathrm{C} 15-\mathrm{C} 16-\mathrm{N} 4$ & $-5.2(6)$ \\
\hline $\mathrm{C} 28-\mathrm{C} 15-\mathrm{C} 16-\mathrm{N} 4$ & $173.2(4)$ \\
\hline $\mathrm{N} 3-\mathrm{C} 15-\mathrm{C} 16-\mathrm{C} 17$ & $170.7(4)$ \\
\hline $\mathrm{C} 28-\mathrm{C} 15-\mathrm{C} 16-\mathrm{C} 17$ & $-10.9(7)$ \\
\hline $\mathrm{N} 4-\mathrm{C} 16-\mathrm{C} 17-\mathrm{C} 18$ & $-3.1(8)$ \\
\hline $\mathrm{C} 15-\mathrm{C} 16-\mathrm{C} 17-\mathrm{C} 18$ & $-178.4(4)$ \\
\hline $\mathrm{N} 4-\mathrm{C} 16-\mathrm{C} 17-\mathrm{C} 22$ & $174.2(5)$ \\
\hline $\mathrm{C} 15-\mathrm{C} 16-\mathrm{C} 17-\mathrm{C} 22$ & $-1.0(7)$ \\
\hline $\mathrm{C} 22-\mathrm{C} 17-\mathrm{C} 18-\mathrm{C} 19$ & $-1.5(8)$ \\
\hline $\mathrm{C} 16-\mathrm{C} 17-\mathrm{C} 18-\mathrm{C} 19$ & $175.9(5)$ \\
\hline $\mathrm{C} 17-\mathrm{C} 18-\mathrm{C} 19-\mathrm{C} 20$ & $-1.3(9)$ \\
\hline $\mathrm{C} 18-\mathrm{C} 19-\mathrm{C} 20-\mathrm{C} 21$ & $2.5(9)$ \\
\hline $\mathrm{C} 19-\mathrm{C} 20-\mathrm{C} 21-\mathrm{C} 22$ & $-1.1(9)$ \\
\hline $\mathrm{C} 20-\mathrm{C} 21-\mathrm{C} 22-\mathrm{C} 17$ & $-1.7(8)$ \\
\hline $\mathrm{C} 20-\mathrm{C} 21-\mathrm{C} 22-\mathrm{C} 23$ & $174.2(5)$ \\
\hline $\mathrm{C} 18-\mathrm{C} 17-\mathrm{C} 22-\mathrm{C} 21$ & $2.9(7)$ \\
\hline $\mathrm{C} 16-\mathrm{C} 17-\mathrm{C} 22-\mathrm{C} 21$ & $-174.6(5)$ \\
\hline $\mathrm{C} 18-\mathrm{C} 17-\mathrm{C} 22-\mathrm{C} 23$ & $-172.9(4)$ \\
\hline $\mathrm{C} 16-\mathrm{C} 17-\mathrm{C} 22-\mathrm{C} 23$ & $9.6(7)$ \\
\hline $\mathrm{C} 21-\mathrm{C} 22-\mathrm{C} 23-\mathrm{C} 24$ & $-5.0(8)$ \\
\hline $\mathrm{C} 17-\mathrm{C} 22-\mathrm{C} 23-\mathrm{C} 24$ & $170.7(5)$ \\
\hline $\mathrm{C} 21-\mathrm{C} 22-\mathrm{C} 23-\mathrm{C} 28$ & $177.8(5)$ \\
\hline $\mathrm{C} 17-\mathrm{C} 22-\mathrm{C} 23-\mathrm{C} 28$ & $-6.6(7)$ \\
\hline $\mathrm{C} 28-\mathrm{C} 23-\mathrm{C} 24-\mathrm{C} 25$ & $2.0(8)$ \\
\hline $\mathrm{C} 22-\mathrm{C} 23-\mathrm{C} 24-\mathrm{C} 25$ & $-175.3(5)$ \\
\hline $\mathrm{C} 23-\mathrm{C} 24-\mathrm{C} 25-\mathrm{C} 26$ & $0.8(9)$ \\
\hline $\mathrm{C} 24-\mathrm{C} 25-\mathrm{C} 26-\mathrm{C} 27$ & $-2.5(9)$ \\
\hline $\mathrm{C} 25-\mathrm{C} 26-\mathrm{C} 27-\mathrm{C} 28$ & $1.4(8)$ \\
\hline $\mathrm{C} 26-\mathrm{C} 27-\mathrm{C} 28-\mathrm{C} 23$ & $1.5(8)$ \\
\hline $\mathrm{C} 26-\mathrm{C} 27-\mathrm{C} 28-\mathrm{C} 15$ & $-178.9(5)$ \\
\hline $\mathrm{C} 24-\mathrm{C} 23-\mathrm{C} 28-\mathrm{C} 27$ & $-3.1(7)$ \\
\hline
\end{tabular}

\begin{tabular}{|c|c|}
\hline $\mathrm{C} 61-\mathrm{C} 62-\mathrm{C} 63-\mathrm{C} 64$ & $0.3(8)$ \\
\hline $\mathrm{C} 60-\mathrm{C} 59-\mathrm{C} 64-\mathrm{C} 63$ & $1.2(7)$ \\
\hline $\mathrm{C} 58-\mathrm{C} 59-\mathrm{C} 64-\mathrm{C} 63$ & $-173.3(4)$ \\
\hline $\mathrm{C} 60-\mathrm{C} 59-\mathrm{C} 64-\mathrm{C} 65$ & $179.8(4)$ \\
\hline $\mathrm{C} 58-\mathrm{C} 59-\mathrm{C} 64-\mathrm{C} 65$ & $5.3(6)$ \\
\hline $\mathrm{C} 62-\mathrm{C} 63-\mathrm{C} 64-\mathrm{C} 59$ & $-0.6(7)$ \\
\hline $\mathrm{C} 62-\mathrm{C} 63-\mathrm{C} 64-\mathrm{C} 65$ & $-179.2(5)$ \\
\hline $\mathrm{C} 59-\mathrm{C} 64-\mathrm{C} 65-\mathrm{C} 66$ & $164.0(5)$ \\
\hline $\mathrm{C} 63-\mathrm{C} 64-\mathrm{C} 65-\mathrm{C} 66$ & $-17.5(7)$ \\
\hline $\mathrm{C} 59-\mathrm{C} 64-\mathrm{C} 65-\mathrm{C} 70$ & $-17.0(7)$ \\
\hline $\mathrm{C} 63-\mathrm{C} 64-\mathrm{C} 65-\mathrm{C} 70$ & $161.6(5)$ \\
\hline $\mathrm{C} 70-\mathrm{C} 65-\mathrm{C} 66-\mathrm{C} 67$ & $0.5(7)$ \\
\hline $\mathrm{C} 64-\mathrm{C} 65-\mathrm{C} 66-\mathrm{C} 67$ & $179.6(4)$ \\
\hline $\mathrm{C} 65-\mathrm{C} 66-\mathrm{C} 67-\mathrm{C} 68$ & $-0.3(8)$ \\
\hline $\mathrm{C} 66-\mathrm{C} 67-\mathrm{C} 68-\mathrm{C} 69$ & $0.0(8)$ \\
\hline $\mathrm{C} 67-\mathrm{C} 68-\mathrm{C} 69-\mathrm{C} 70$ & $0.0(8)$ \\
\hline $\mathrm{C} 68-\mathrm{C} 69-\mathrm{C} 70-\mathrm{C} 65$ & $0.2(8)$ \\
\hline $\mathrm{C} 68-\mathrm{C} 69-\mathrm{C} 70-\mathrm{C} 57$ & $178.7(5)$ \\
\hline $\mathrm{C} 66-\mathrm{C} 65-\mathrm{C} 70-\mathrm{C} 69$ & $-0.5(7)$ \\
\hline $\mathrm{C} 64-\mathrm{C} 65-\mathrm{C} 70-\mathrm{C} 69$ & $-179.5(4)$ \\
\hline $\mathrm{C} 66-\mathrm{C} 65-\mathrm{C} 70-\mathrm{C} 57$ & $-179.1(4)$ \\
\hline $\mathrm{C} 64-\mathrm{C} 65-\mathrm{C} 70-\mathrm{C} 57$ & $1.9(7)$ \\
\hline $\mathrm{N} 9-\mathrm{C} 57-\mathrm{C} 70-\mathrm{C} 69$ & $24.8(8)$ \\
\hline $\mathrm{C} 58-\mathrm{C} 57-\mathrm{C} 70-\mathrm{C} 69$ & $-155.6(5)$ \\
\hline N9-C57-C70-C65 & $-156.6(5)$ \\
\hline $\mathrm{C} 58-\mathrm{C} 57-\mathrm{C} 70-\mathrm{C} 65$ & $23.0(6)$ \\
\hline $\mathrm{C} 2-\mathrm{C} 1-\mathrm{N} 1-\mathrm{O} 1$ & $-173.7(4)$ \\
\hline $\mathrm{C} 14-\mathrm{C} 1-\mathrm{N} 1-\mathrm{O} 1$ & $6.0(7)$ \\
\hline $\mathrm{C} 2-\mathrm{C} 1-\mathrm{N} 1-\mathrm{Ni} 1$ & $23.2(6)$ \\
\hline $\mathrm{C} 14-\mathrm{C} 1-\mathrm{N} 1-\mathrm{Ni} 1$ & $-157.2(4)$ \\
\hline $\mathrm{C} 3-\mathrm{C} 2-\mathrm{N} 2-\mathrm{O} 2$ & $-175.1(4)$ \\
\hline $\mathrm{C} 1-\mathrm{C} 2-\mathrm{N} 2-\mathrm{O} 2$ & $5.3(7)$ \\
\hline $\mathrm{C} 28-\mathrm{C} 15-\mathrm{N} 3-\mathrm{O} 3$ & $2.9(7)$ \\
\hline $\mathrm{C} 16-\mathrm{C} 15-\mathrm{N} 3-\mathrm{O} 3$ & $-178.9(4)$ \\
\hline $\mathrm{C} 28-\mathrm{C} 15-\mathrm{N} 3-\mathrm{Ni} 2$ & $-176.9(4)$ \\
\hline $\mathrm{C} 16-\mathrm{C} 15-\mathrm{N} 3-\mathrm{Ni} 2$ & $1.4(5)$ \\
\hline $\mathrm{C} 17-\mathrm{C} 16-\mathrm{N} 4-\mathrm{O} 4$ & $9.2(7)$ \\
\hline $\mathrm{C} 15-\mathrm{C} 16-\mathrm{N} 4-\mathrm{O} 4$ & $-175.3(4)$ \\
\hline $\mathrm{C} 17-\mathrm{C} 16-\mathrm{N} 4-\mathrm{Ni} 2$ & $-168.8(4)$ \\
\hline $\mathrm{C} 15-\mathrm{C} 16-\mathrm{N} 4-\mathrm{Ni} 2$ & $6.8(5)$ \\
\hline $\mathrm{C} 30-\mathrm{C} 29-\mathrm{N} 5-\mathrm{O} 5$ & $-178.1(4)$ \\
\hline $\mathrm{C} 42-\mathrm{C} 29-\mathrm{N} 5-\mathrm{O} 5$ & $0.8(8)$ \\
\hline $\mathrm{C} 30-\mathrm{C} 29-\mathrm{N} 5-\mathrm{Ni} 1$ & $1.0(5)$ \\
\hline $\mathrm{C} 42-\mathrm{C} 29-\mathrm{N} 5-\mathrm{Ni} 1$ & $179.9(4)$ \\
\hline $\mathrm{C} 31-\mathrm{C} 30-\mathrm{N} 6-\mathrm{O} 6$ & $1.7(7)$ \\
\hline $\mathrm{C} 29-\mathrm{C} 30-\mathrm{N} 6-\mathrm{O} 6$ & $-178.3(4)$ \\
\hline $\mathrm{C} 31-\mathrm{C} 30-\mathrm{N} 6-\mathrm{Ni} 1$ & $-178.0(4)$ \\
\hline $\mathrm{C} 29-\mathrm{C} 30-\mathrm{N} 6-\mathrm{Ni} 1$ & $2.0(5)$ \\
\hline
\end{tabular}




\begin{tabular}{|c|c|}
\hline $\mathrm{C} 22-\mathrm{C} 23-\mathrm{C} 28-\mathrm{C} 27$ & $174.2(5)$ \\
\hline $\mathrm{C} 24-\mathrm{C} 23-\mathrm{C} 28-\mathrm{C} 15$ & $177.3(5)$ \\
\hline $\mathrm{C} 22-\mathrm{C} 23-\mathrm{C} 28-\mathrm{C} 15$ & $-5.4(7)$ \\
\hline $\mathrm{N} 3-\mathrm{C} 15-\mathrm{C} 28-\mathrm{C} 27$ & $12.4(8)$ \\
\hline $\mathrm{C} 16-\mathrm{C} 15-\mathrm{C} 28-\mathrm{C} 27$ & $-165.7(5)$ \\
\hline $\mathrm{N} 3-\mathrm{C} 15-\mathrm{C} 28-\mathrm{C} 23$ & $-168.0(5)$ \\
\hline $\mathrm{C} 16-\mathrm{C} 15-\mathrm{C} 28-\mathrm{C} 23$ & $13.9(7)$ \\
\hline $\mathrm{N} 5-\mathrm{C} 29-\mathrm{C} 30-\mathrm{N} 6$ & $-1.9(6)$ \\
\hline $\mathrm{C} 42-\mathrm{C} 29-\mathrm{C} 30-\mathrm{N} 6$ & $179.1(4)$ \\
\hline $\mathrm{N} 5-\mathrm{C} 29-\mathrm{C} 30-\mathrm{C} 31$ & $178.1(4)$ \\
\hline $\mathrm{C} 42-\mathrm{C} 29-\mathrm{C} 30-\mathrm{C} 31$ & $-0.9(7)$ \\
\hline $\mathrm{N} 6-\mathrm{C} 30-\mathrm{C} 31-\mathrm{C} 32$ & $-0.6(8)$ \\
\hline $\mathrm{C} 29-\mathrm{C} 30-\mathrm{C} 31-\mathrm{C} 32$ & $179.4(5)$ \\
\hline $\mathrm{N} 6-\mathrm{C} 30-\mathrm{C} 31-\mathrm{C} 36$ & $179.1(5)$ \\
\hline $\mathrm{C} 29-\mathrm{C} 30-\mathrm{C} 31-\mathrm{C} 36$ & $-0.9(7)$ \\
\hline $\mathrm{C} 36-\mathrm{C} 31-\mathrm{C} 32-\mathrm{C} 33$ & $2.2(8)$ \\
\hline $\mathrm{C} 30-\mathrm{C} 31-\mathrm{C} 32-\mathrm{C} 33$ & $-178.1(5)$ \\
\hline $\mathrm{C} 31-\mathrm{C} 32-\mathrm{C} 33-\mathrm{C} 34$ & $-1.0(9)$ \\
\hline $\mathrm{C} 32-\mathrm{C} 33-\mathrm{C} 34-\mathrm{C} 35$ & $-0.1(10)$ \\
\hline $\mathrm{C} 33-\mathrm{C} 34-\mathrm{C} 35-\mathrm{C} 36$ & $0.0(10)$ \\
\hline $\mathrm{C} 34-\mathrm{C} 35-\mathrm{C} 36-\mathrm{C} 31$ & $1.2(8)$ \\
\hline $\mathrm{C} 34-\mathrm{C} 35-\mathrm{C} 36-\mathrm{C} 37$ & $179.5(6)$ \\
\hline $\mathrm{C} 32-\mathrm{C} 31-\mathrm{C} 36-\mathrm{C} 35$ & $-2.2(7)$ \\
\hline $\mathrm{C} 30-\mathrm{C} 31-\mathrm{C} 36-\mathrm{C} 35$ & $178.1(5)$ \\
\hline $\mathrm{C} 32-\mathrm{C} 31-\mathrm{C} 36-\mathrm{C} 37$ & $179.4(4)$ \\
\hline $\mathrm{C} 30-\mathrm{C} 31-\mathrm{C} 36-\mathrm{C} 37$ & $-0.3(7)$ \\
\hline $\mathrm{C} 35-\mathrm{C} 36-\mathrm{C} 37-\mathrm{C} 38$ & $4.1(7)$ \\
\hline $\mathrm{C} 31-\mathrm{C} 36-\mathrm{C} 37-\mathrm{C} 38$ & $-177.6(4)$ \\
\hline $\mathrm{C} 35-\mathrm{C} 36-\mathrm{C} 37-\mathrm{C} 42$ & $-174.7(5)$ \\
\hline $\mathrm{C} 31-\mathrm{C} 36-\mathrm{C} 37-\mathrm{C} 42$ & $3.6(7)$ \\
\hline $\mathrm{C} 42-\mathrm{C} 37-\mathrm{C} 38-\mathrm{C} 39$ & $1.9(8)$ \\
\hline $\mathrm{C} 36-\mathrm{C} 37-\mathrm{C} 38-\mathrm{C} 39$ & $-177.0(5)$ \\
\hline $\mathrm{C} 37-\mathrm{C} 38-\mathrm{C} 39-\mathrm{C} 40$ & $-0.1(9)$ \\
\hline $\mathrm{C} 38-\mathrm{C} 39-\mathrm{C} 40-\mathrm{C} 41$ & $-0.6(8)$ \\
\hline $\mathrm{C} 39-\mathrm{C} 40-\mathrm{C} 41-\mathrm{C} 42$ & $-0.6(8)$ \\
\hline $\mathrm{C} 40-\mathrm{C} 41-\mathrm{C} 42-\mathrm{C} 37$ & $2.4(8)$ \\
\hline $\mathrm{C} 40-\mathrm{C} 41-\mathrm{C} 42-\mathrm{C} 29$ & $-176.4(5)$ \\
\hline $\mathrm{C} 38-\mathrm{C} 37-\mathrm{C} 42-\mathrm{C} 41$ & $-3.0(7)$ \\
\hline $\mathrm{C} 36-\mathrm{C} 37-\mathrm{C} 42-\mathrm{C} 41$ & $175.8(4)$ \\
\hline $\mathrm{C} 38-\mathrm{C} 37-\mathrm{C} 42-\mathrm{C} 29$ & $175.9(4)$ \\
\hline $\mathrm{C} 36-\mathrm{C} 37-\mathrm{C} 42-\mathrm{C} 29$ & $-5.2(7)$ \\
\hline $\mathrm{N} 5-\mathrm{C} 29-\mathrm{C} 42-\mathrm{C} 41$ & $4.0(8)$ \\
\hline $\mathrm{C} 30-\mathrm{C} 29-\mathrm{C} 42-\mathrm{C} 41$ & $-177.2(4)$ \\
\hline $\mathrm{N} 5-\mathrm{C} 29-\mathrm{C} 42-\mathrm{C} 37$ & $-174.9(5)$ \\
\hline $\mathrm{C} 30-\mathrm{C} 29-\mathrm{C} 42-\mathrm{C} 37$ & $3.9(7)$ \\
\hline $\mathrm{N} 7-\mathrm{C} 43-\mathrm{C} 44-\mathrm{N} 8$ & $-29.8(8)$ \\
\hline $\mathrm{C} 56-\mathrm{C} 43-\mathrm{C} 44-\mathrm{N} 8$ & $151.8(5)$ \\
\hline $\mathrm{N} 7-\mathrm{C} 43-\mathrm{C} 44-\mathrm{C} 45$ & $150.5(5)$ \\
\hline
\end{tabular}

$\begin{array}{ll}\text { C56-C43-N7-O7 } & 2.8(8) \\ \text { C44-C43-N7-O7 } & -175.3(4) \\ \text { C56-C43-N7-Ni2 } & -160.1(4) \\ \text { C44-C43-N7-Ni2 } & 21.8(7) \\ \text { C43-C44-N8-O8 } & 4.6(8) \\ \text { C45-C44-N8-O8 } & -175.8(4) \\ \text { C70-C57-N9-O9 } & 0.4(8) \\ \text { C58-C57-N9-O9 } & -179.2(4) \\ \text { C70-C57-N9-Ni3 } & -162.4(4) \\ \text { C58-C57-N9-Ni3 } & 18.0(5) \\ \text { C59-C58-N10-O10 } & 7.1(8) \\ \text { C57-C58-N10-O10 } & 179.3(4) \\ \text { C59-C58-N10-Ni3 } & -147.0(4) \\ \text { C57-C58-N10-Ni3 } & 25.2(5) \\ \text { C1-N1-O1-B1 } & 137.4(5) \\ \text { Ni1-N1-O1-B1 } & -57.6(5) \\ \text { C2-N2-O2-Ni1 } & 32.8(6) \\ \text { C2-N2-O2-Ni3 } & -142.9(4) \\ \text { C15-N3-O3-B2 } & -175.3(5) \\ \text { Ni2-N3-O3-B2 } & 4.4(7) \\ \text { C16-N4-O4-Ni3 } & -145.0(4) \\ \text { Ni2-N4-O4-Ni3 } & 32.9(4) \\ \text { C29-N5-O5-B1 } & -173.4(5) \\ \text { Ni1-N5-O5-B1 } & 7.6(7) \\ \text { C30-N6-O6-Ni3 } & -146.0(4) \\ \text { Ni1-N6-O6-Ni3 } & 33.6(4) \\ \text { C43-N7-O7-B2 } & 139.1(5) \\ \text { Ni2-N7-O7-B2 } & -55.7(5) \\ \text { C44-N8-O8-Ni2 } & 29.2(6) \\ \text { C44-N8-O8-Ni3 } & -146.7(4) \\ \text { N2-O2-Ni1-N6 } & 152.7(4) \\ \text { Ni3-O2-Ni1-N6 } & -31.5(2) \\ \text { N2-O2-Ni1-N1 } & -34.0(4) \\ \text { Ni3-O2-Ni1-N1 } & 141.8(2) \\ \text { C29-N5-Ni1-N6 } & 0.0(4) \\ \text { O5-N5-Ni1-N6 } & 179.0(4) \\ \text { C29-N5-Ni1-N1 } & -173.2(4) \\ \text { O5-N5-Ni1-N1 } & 5.8(4) \\ \text { C30-N6-Ni1-O2 } & 177.9(4) \\ \text { O6-N6-Ni1-O2 } & -1.7(3) \\ \text { C30-N6-Ni1-N5 } & -1.2(4) \\ \text { O6-N6-Ni1-N5 } & 179.1(4) \\ \text { C1-N1-Ni1-O2 } & 4.2(4) \\ \text { O1-N1-Ni1-O2 } & -159.1(3) \\ \text { C1-N1-Ni1-N5 } & -177.8(4) \\ \text { O1-N1-Ni1-N5 } & \\ \text { N8-O8-Ni2-N4 } & \\ \text { Ni3-O8-Ni2-N4 } & (2) \\ & \end{array}$




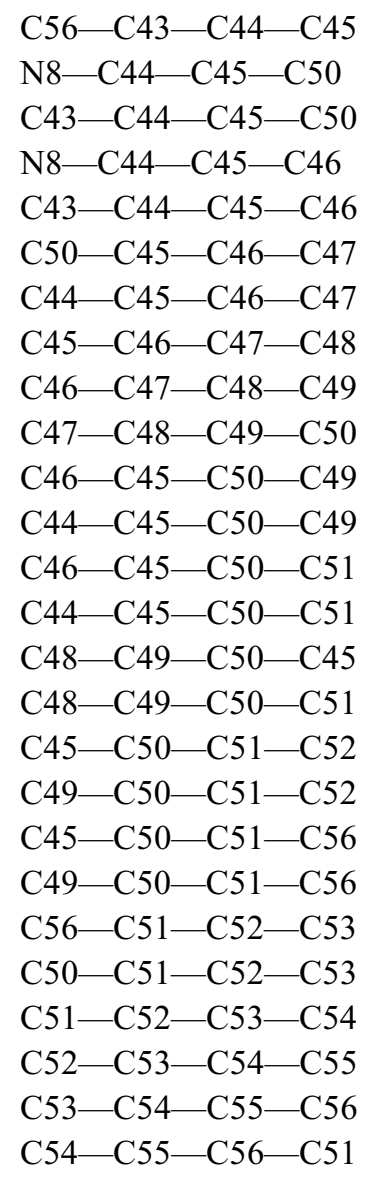

$-27.8(7)$
$-161.0(5)$
$18.7(7)$
$18.7(8)$
$-161.6(5)$
$-0.2(9)$
$-179.9(6)$
$-0.4(11)$
$0.8(12)$
$-0.6(12)$
$0.4(9)$
$-179.9(5)$
$-178.3(5)$
$1.4(8)$
$0.0(10)$
$178.7(7)$
$166.2(5)$
$-12.4(9)$
$-12.1(8)$
$169.3(6)$
$-0.8(9)$
$-179.1(6)$
$2.5(10)$
$-1.9(10)$
$-0.4(9)$
$2.1(8)$

$\mathrm{N} 8-\mathrm{O} 8-\mathrm{Ni} 2-\mathrm{N} 7$

$\mathrm{Ni3}-\mathrm{O} 8-\mathrm{Ni} 2-\mathrm{N} 7$

$\mathrm{C} 15-\mathrm{N} 3-\mathrm{Ni} 2-\mathrm{N} 4$

$\mathrm{O} 3-\mathrm{N} 3-\mathrm{Ni} 2-\mathrm{N} 4$

$\mathrm{C} 15-\mathrm{N} 3-\mathrm{Ni} 2-\mathrm{N} 7$

$\mathrm{O} 3-\mathrm{N} 3-\mathrm{Ni} 2-\mathrm{N} 7$

$\mathrm{O} 4-\mathrm{N} 4-\mathrm{Ni} 2-\mathrm{O} 8$

C16- $44-\mathrm{Ni} 2-\mathrm{O} 8$

$\mathrm{O} 4-\mathrm{N} 4-\mathrm{Ni} 2-\mathrm{N} 3$

$\mathrm{C} 16-\mathrm{N} 4-\mathrm{Ni} 2-\mathrm{N} 3$

$\mathrm{C} 43-\mathrm{N} 7-\mathrm{Ni} 2-\mathrm{O} 8$

$\mathrm{O} 7-\mathrm{N} 7-\mathrm{Ni} 2-\mathrm{O} 8$

$\mathrm{C} 43-\mathrm{N} 7-\mathrm{Ni} 2-\mathrm{N} 3$

$\mathrm{O} 7-\mathrm{N} 7-\mathrm{Ni} 2-\mathrm{N} 3$

$\mathrm{N} 5-\mathrm{O} 5-\mathrm{B} 1-\mathrm{F} 2$

$\mathrm{N} 5-\mathrm{O} 5-\mathrm{B} 1-\mathrm{F} 1$

N5-O5-B1-O1

$\mathrm{N} 1-\mathrm{O} 1-\mathrm{B} 1-\mathrm{F} 2$

$\mathrm{N} 1-\mathrm{O} 1-\mathrm{B} 1-\mathrm{F} 1$

$\mathrm{N} 1-\mathrm{O} 1-\mathrm{B} 1-\mathrm{O} 5$

$\mathrm{N} 3-\mathrm{O} 3-\mathrm{B} 2-\mathrm{F} 4$

$\mathrm{N} 3-\mathrm{O} 3-\mathrm{B} 2-\mathrm{F} 3$

$\mathrm{N} 3-\mathrm{O} 3-\mathrm{B} 2-\mathrm{O} 7$

$\mathrm{N} 7-\mathrm{O} 7-\mathrm{B} 2-\mathrm{F} 4$

$\mathrm{N} 7-\mathrm{O} 7-\mathrm{B} 2-\mathrm{F} 3$

$\mathrm{N} 7-\mathrm{O} 7-\mathrm{B} 2-\mathrm{O} 3$
$-29.5(4)$

146.4 (2)

$1.7(4)$

$-178.0(4)$

$-171.6(4)$

$8.7(4)$

$-3.9(3)$

$174.1(4)$

$177.1(4)$

-4.9 (3)

$2.3(5)$

$-161.0(3)$

$-179.8(5)$

16.9 (4)

$-161.0(5)$

$76.6(7)$

$-45.0(7)$

$-170.9(4)$

$-49.6(6)$

$73.6(6)$

$80.1(6)$

$-158.4(5)$

$-42.7(7)$

$-50.1(6)$

$-171.4(4)$

$73.4(6)$

Hydrogen-bond geometry $\left(A,{ }^{\circ}\right)$

\begin{tabular}{lllll}
\hline$D-\mathrm{H} \cdots A$ & $D-\mathrm{H}$ & $\mathrm{H} \cdots A$ & $D \cdots A$ & $D-\mathrm{H} \cdots A$ \\
\hline $\mathrm{O} 9-\mathrm{H} 1 A \cdots \mathrm{N} 2$ & 0.85 & 1.96 & $2.771(6)$ & 158 \\
$\mathrm{O} 10-\mathrm{H} 2 A \cdots \mathrm{N} 8$ & 1.06 & 1.77 & $2.765(6)$ & 155 \\
\hline
\end{tabular}

